\title{
Wrernen ay wat
}

\section{FINANCIAL CONSTRAINTS IN CAPACITY PLANNING: \\ A NATIONAL UTILITY REGULATORY MODEL (NUREG)}

Volume III of III:

Software Description

FINAL REPORT DO WOF WOROFHM

Submitted to:

Division of Coal and Electric Power

Energy Information Administration

U.S. Department of Energy

Prepared Under Contract No. DEAC-01-79EI-10579

October 29, 1981 


\section{DISCLAIMER}

This report was prepared as an account of work sponsored by an agency of the United States Government. Neither the United States Government nor any agency Thereof, nor any of their employees, makes any warranty, express or implied, or assumes any legal liability or responsibility for the accuracy, completeness, or usefulness of any information, apparatus, product, or process disclosed, or represents that its use would not infringe privately owned rights. Reference herein to any specific commercial product, process, or service by trade name, trademark, manufacturer, or otherwise does not necessarily constitute or imply its endorsement, recommendation, or favoring by the United States Government or any agency thereof. The views and opinions of authors expressed herein do not necessarily state or reflect those of the United States Government or any agency thereof. 


\section{DISCLAIMER}

Portions of this document may be illegible in electronic image products. Images are produced from the best available original document. 


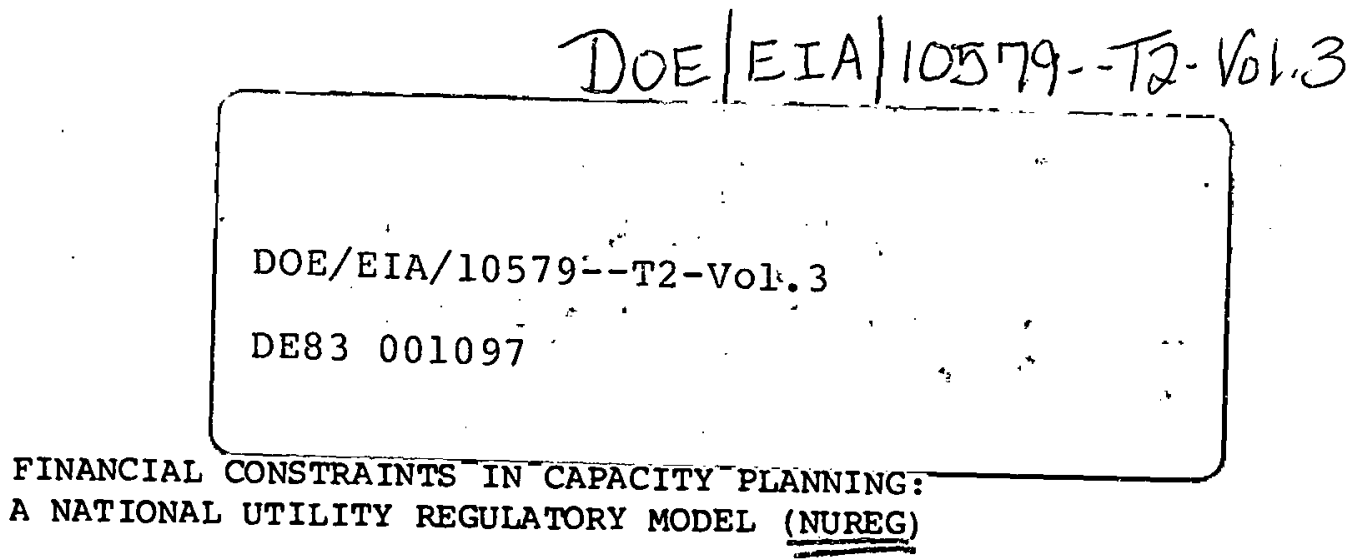

VOLUME III OF III: SOFTWARE DESCRIPTION

Final Report

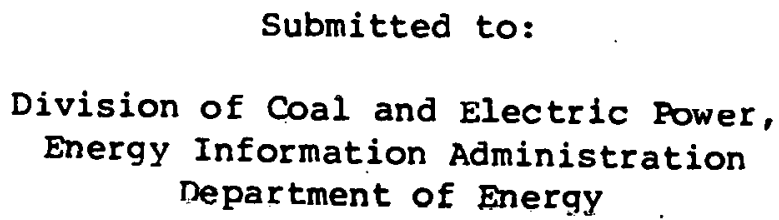

NOTICE

ICF Incorporated

This report was prepared as an account of work sponsored by an agency of the United States Government Neiiner the United States Governmient nor any agency thereot. nor any of their emplovees. makes any warranty, express of imolied, or assumes any legal liability of responsibility tor the occuracy,
compleieness. or ussfuliness of any information, apparatus, product, or process disclosed, or represents that its use would not intringe privately owned rights. Reference herein to any specilic commercial product, process, of service by, trade nams. trademark, manufacturer, or otherwise, does not necessarily constitute or imply its endersement. recommendation. or tavoring by the United States Government or any ageney thereof. The views and opinions of authors expressed herein do not

PORTIONS OF THIS REPORT ARE ILLEGIBLE. It

has been reproduced from the best àvallable

copy to permit tho broadest possible avail-

ability.

October 29, 1981 
This volume is the software description for the National Utility Regulatory Model (NUREG). This is the third of three volumes provided by ICF under contract number DEAC-01-79EI-10579. These three volumes are:

- a manual describing the NUREG methodology,

- a users guide, and

- a description of the software.

This manual describes the software which has been developed for NUREG. This includes a listing of the source modules. All computer code has been written in FORTRAN. 
PAGE

1.0 INTRODUCTION 1

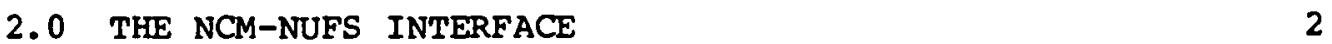

3.0 THE FINANCIAL SENSITIVITY PROGRAM (FSP) 19

4.0 THE CAPACITY ADDITIONS ADJUSTMENT PROGRAM (CAAP) 29

$\begin{array}{lll}5.0 & \text { NUREG.FORMI.FORT } & 38\end{array}$

$\begin{array}{lll}6.0 & \text { NUREG. FORMIM.FORT } & 62\end{array}$ 


\subsection{INTRODUCTION}

This document contains listings and descriptions of the programs developed for the NUREG methodology. The programs described in this document are:

- The NCM-NUFS Interface

- The Financial Sensitivity Program (FSP)

- The Capacity Additions Adjustments Program (CAAP)

- NUREG.FORMI.FORT

- NUREG. FORMIM.FORT

The later two programs are used to generate historical financial data for NuFs aggregated to the NCM regions. Note that descriptions of NUFS and NCM are not contained in this volume as they are fully documented elsewhere. 


\subsection{THE NCM-NUFS INTERFACE}

This program reads the National Coal Model solution files, extracts and processes the information needed for NUFS, and writes this information to a partioned data set. The input files needed are:

- the NCM solution files for 1985, 1990, and 1995,

- the binary file that contains the common block data from NCM, and

- the historical data file containing 1979 generation data.

The units numbers for these files are shown in Table 1. In addition, the code links to a library that contains several routines that are used to compare and manipulate character strings. This library is also used by NCM.

TABLE 1

INPUT FILES FOR THE NCM-NUFS INTERFACE

File $\quad$ Unit Number

$\begin{array}{ll}1985 \text { NCM Solution } & 25 \\ 1990 \text { NCM Solution } & 26 \\ 1995 \text { NCM Solution } & 27 \\ \text { Common Block Data } & 13 \\ \text { Historical Data } & 98\end{array}$

Figure 1 shows the organization of the program.

FIGURE 1

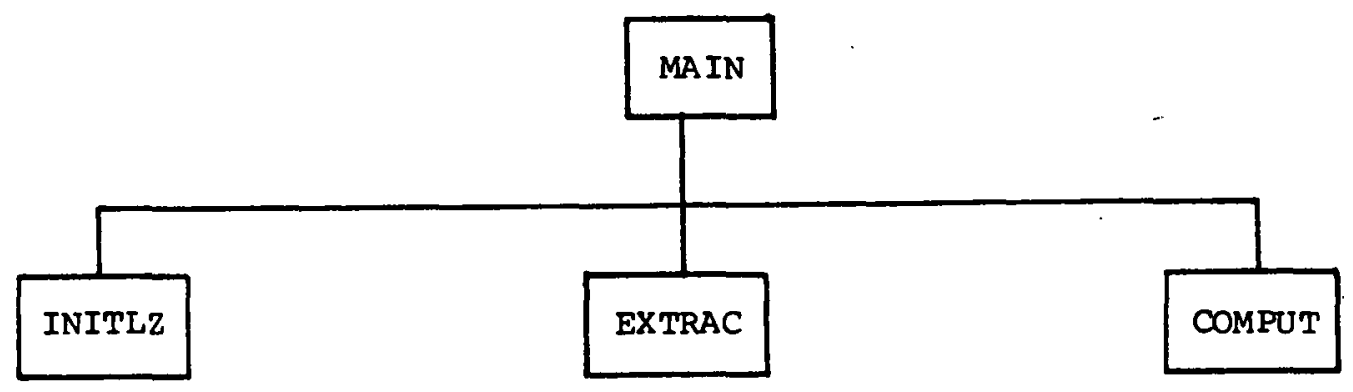


The main program first calls subroutine INITLZ. This subroutine reads the common block and historical data. It also sets up arrays that are used in other portions of the program to aggregate plant types. Subroutine EXTRAC is then called for each run year $(1985,1990$, and 1995). This routine extracts the required information about builds, operates and fuel costs, and sorts and aggregates this information. In addition; EXTRAC calculates power transfers between regions and generation losses.

Finally, subroutine compuT is called. This subroutine calculates builds, capital costs and generation by region. These numbers, as well as fuel costs, are interpolated between run years. In addition, builds are extrapolated from 1995 to 2005. Subroutine COMPUT writes the results of these calculations to temporary files (units 30-74). Additional job steps are used to reassemble these files into a partitioned data set that is read by NUFS. Figure 2 is a listing of the code and control statements.

A listing of the program now follows. 


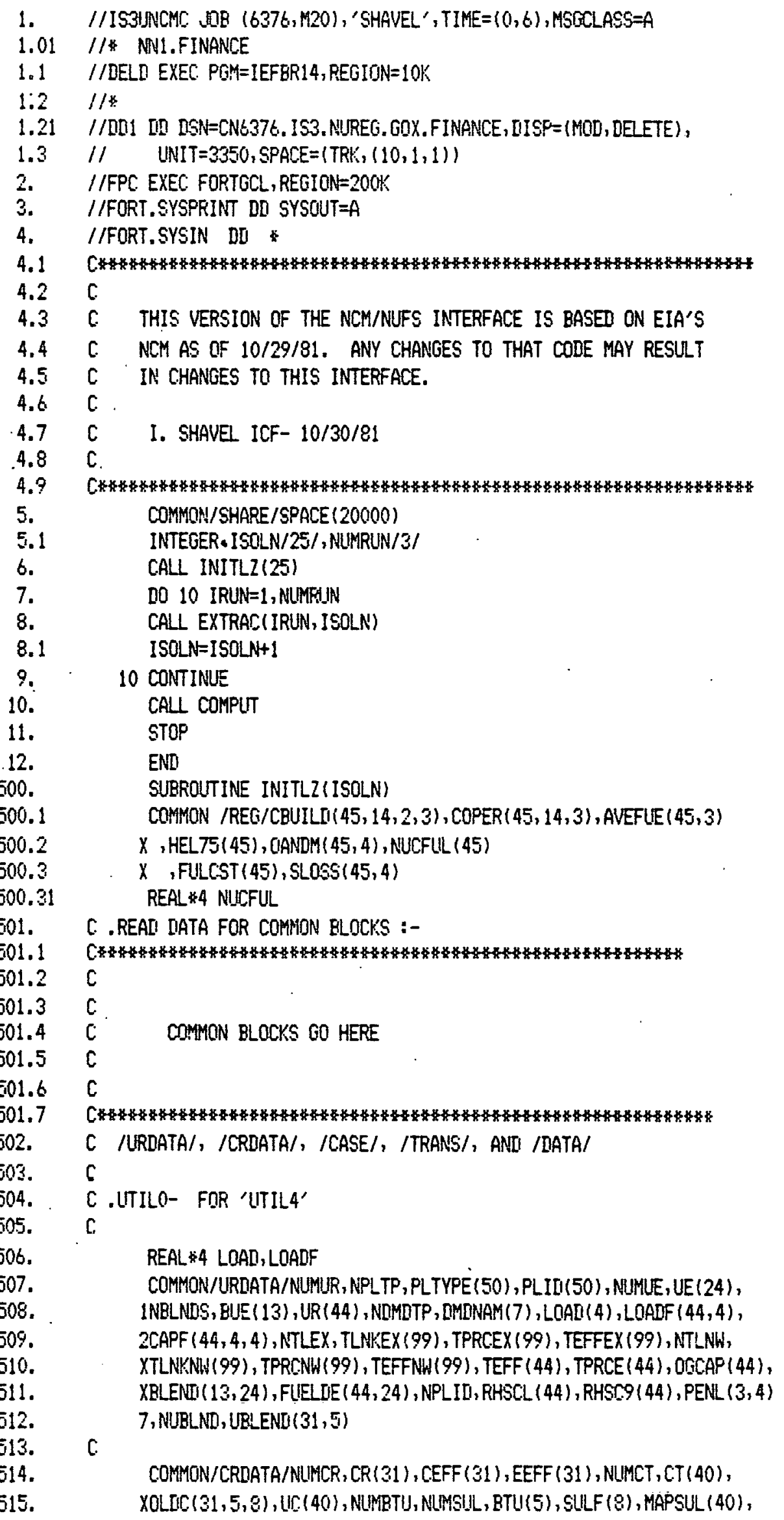


516. XBTUCON $(31,5)$, MSULU, SULU( 6$),$ COUNT $(31,5,8)$

517. $C$

513. REAL*8 CASNAM LIRNAM, CRNAM, CENNAM, PIENAM

519. INTEGER $\$ 4$ TLEYR, BYRCST, EYRPRI, EYREL, BYRCL, BYRPO, EYKOG, CASYF,

$520 . \quad X \quad$ REPTYR, CUALYR

521. COAMON/CASE/CAGNAM(4), URNAM $(44,3), \operatorname{CRNAM}(31,3), \operatorname{CENNAM}(10,3)$,

522. XPIENAM $(12,3)$, CAPLIM, BLDMAX, ELDMIN, UDC, GNF, BYRCST, BYRPRD, EYFEL,

523. XBYRCL, BYFPG, BYFOG, CASYR, SCRCAP, DRGCAP, CCR (44), TLBSW, TLEYR, SHIP,

524. XTIMESW, NYRCST, TRELNW, CSTMLT, REPTYR, REPDEF, COALYR, ULF, CCNM(31),

525. XCCVL (31), NCCRB, NLMCEN, NUMPIE, MUURCEN(44), MCRPIE(31)

551. C

551.1 C C

$551.2 \quad C$

$551.3 \mathrm{C}$

$551.4 \quad C \quad$ END GMS COMMON BLOCKS

$551.5 \quad C$

$551.6 \quad C$

551.7 С C

552. C

553. $C$

554. $C$

556. $C$

558. $C$

559. REAL 8 PBNAME

560. COMMON/FRUN/PENAME, RUNYR

$560.1 \quad C$

560.2 COMMON /HISTOR/ HGN(44)

560.3 REAL $\$ 4$ HGN

561. C

562. COMMON /MAPS/IBMAP (50), NIBMAP, OMAPS(50), IOMAPS(50), NOMAPS

564. INTEGER GDIATA/13/,SOLN/15/

565. C

565.1 C

$565.2 \quad C$

$555.3 \quad C$

$565.4 \quad C$

$565.5 \quad 6$

$565.6 \quad C$

565.7 C

566. $\quad C$

567. DIMENSION BIN1 $(3500)$, BIN2 $(2000)$, BIN3 $(800)$

568. $\quad C$

569. EQUIVALENCE (BIN1, NUMUR), (BIN2, NUMCR), (BIN3, CASNAM)

570. C

571. C.REAII'GDDATA' BINARY FILE

572. $C$

573. REHIND GDDATA

574. READ(GDDATA) BINI

575. READ(GDDATA) BIN2

576. READ(GDDATA) BIN3

$581.01 \quad 6$

581.02 C.4\$

$581.03 \quad \mathrm{C}$

$581.04 \quad C$

581.05 C. FNI NF GIATA READ SECTION

$581.06 \quad \mathrm{C}$ 
$581.07 \quad 0$

581.08 C

581.7

581.8 READ $(5,666)$ NIBMAP

581.9

591.91

581.92

581.93

581.94

581.95

581.96

581.97

582. 666. FORMAT(I2)

I0 201 II $=1$, NIEMAP

READ(5,656) IBMAP(II)

201 CONTINUE

READ $(5,666)$ NOMAPS

DO $20211=1$, NOMAPS

READ (5, 667) OMAPS (II), IOMAPS (II)

202 CONTINUE

667 FORMAT $(A 2,2 X, 12)$

582.1 C READ THE SOLUTION FILE

582.2 C FIX THE READ STATEMENT

583. : REAL *8 ACTD, DUALD, NAME, LBD, UBD

584. REWIND ISOLN

585. C. DO $501 \quad I=1,4$

586. C READ(ISOLN) ACTD

587. C 501 CONTINUE

587.1 FIX THE READ STATEMENT

588. C READ(ISOLN) ACTD, PBNAME, (ACTD, $\downarrow 3,10$ ), FUNCT

589. DO $503 \mathrm{I}=1,9$

5\%. READ(ISOLN) ACTD

591. 503 CONTINUE

591.1. C****READ THE SOLUTION FILE

591.2 CIX THE READ STATEMENT

592. 510 READ(ISOLN, END=530) ACTD, LBD, UBD, DUALD, NAME

593. IF (ICOMP('LDUMYY', NAME, $6,1,1)$.NE, 0) GO TO 510

594. RUNYR=ACTD

595. REHIND ISOLN

596. $\quad$ GO TO 600

597. 530 PRINT 540

598. 540 FORMATI' NO LDIMMY')

600. $C$

600.001600 CALL RDHIST

$600.01 \quad$ NATION=NUMLLR+1

$600.1 \quad$ DO $605 \mathrm{I}=1$, NATION

$600.2 \quad 605 \mathrm{HE} 75(I)=0$.

601. ID $610 \mathrm{I}=1$, NUMUR

601.1 C KREG=FURCEN(I)

$601.2 \quad$ HEL 75 (I) $=$ HGN (I) \& $T E F F(I)$

601.3610 HEL 75 (NATION) $=$ HEL 75 (NATION) +HGN(I) $*$ TEFF (I)

601.4 RETUFN

602. END

1000. SUBROUTINE EXTRAC(IRIN, ISOLN)

1001. C.

1001.1 C

$1001.2 \quad C$

$1001.3 \quad C$

1001.4 C I INSERT GMG COMMON BLOCKS HERE

$1001.5 \quad C$

1001.6 C

1001.7 [

1002. REAL $* 4$ LOAII, LQADF

1003. COMMON/URDATA/NIMLR, NPLTP, PLTYPE(50), PLID(50), NUMUE, UE(24), 


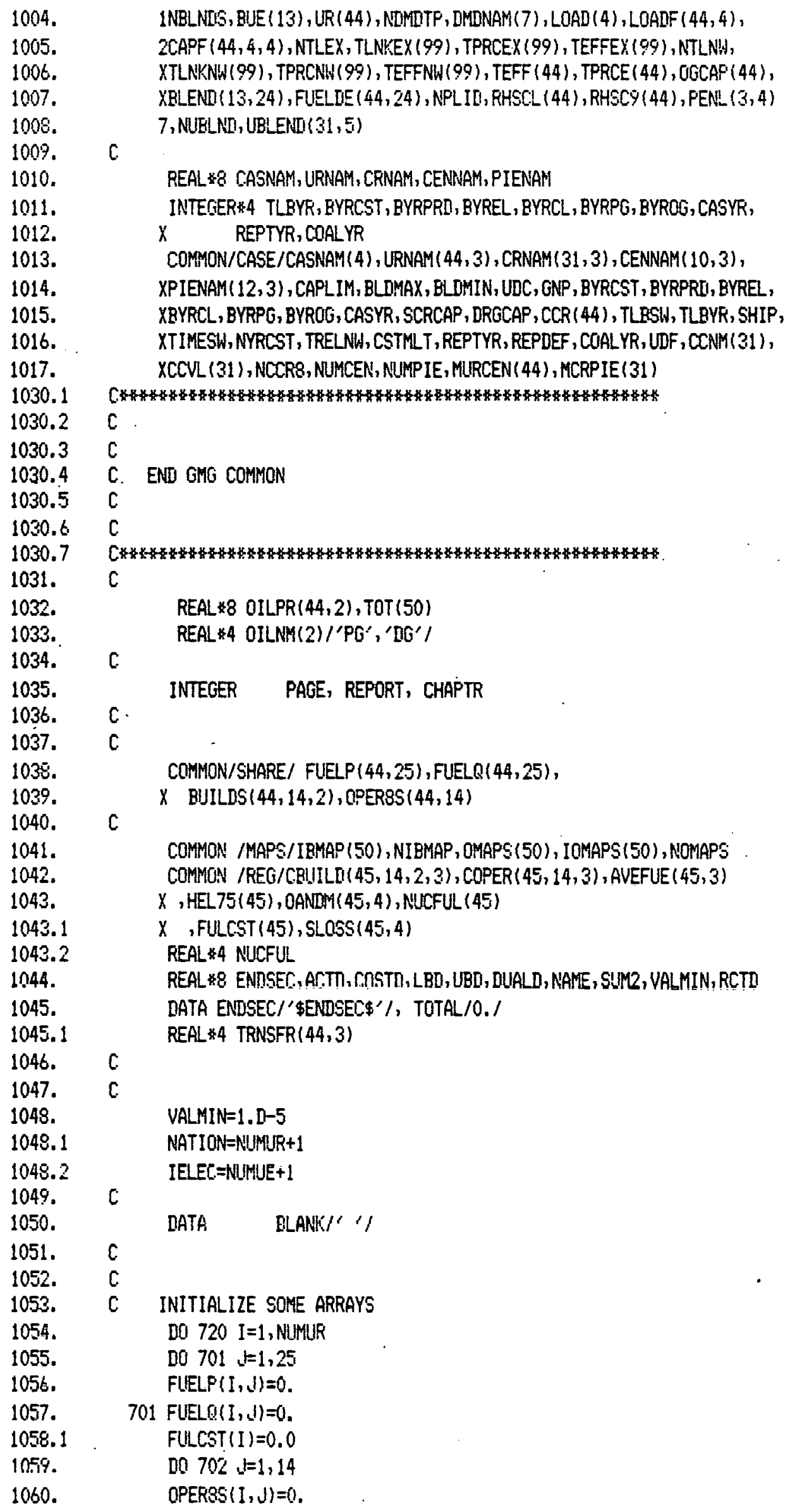




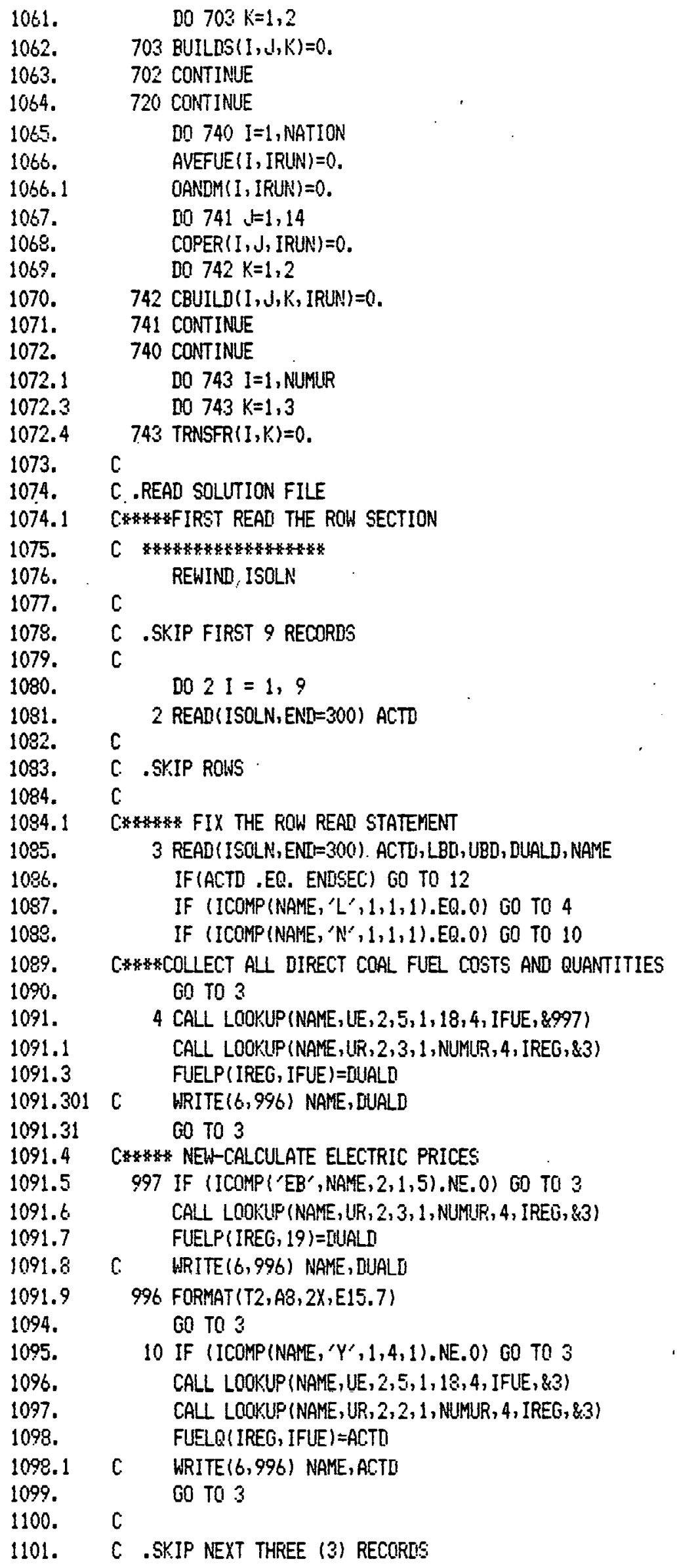




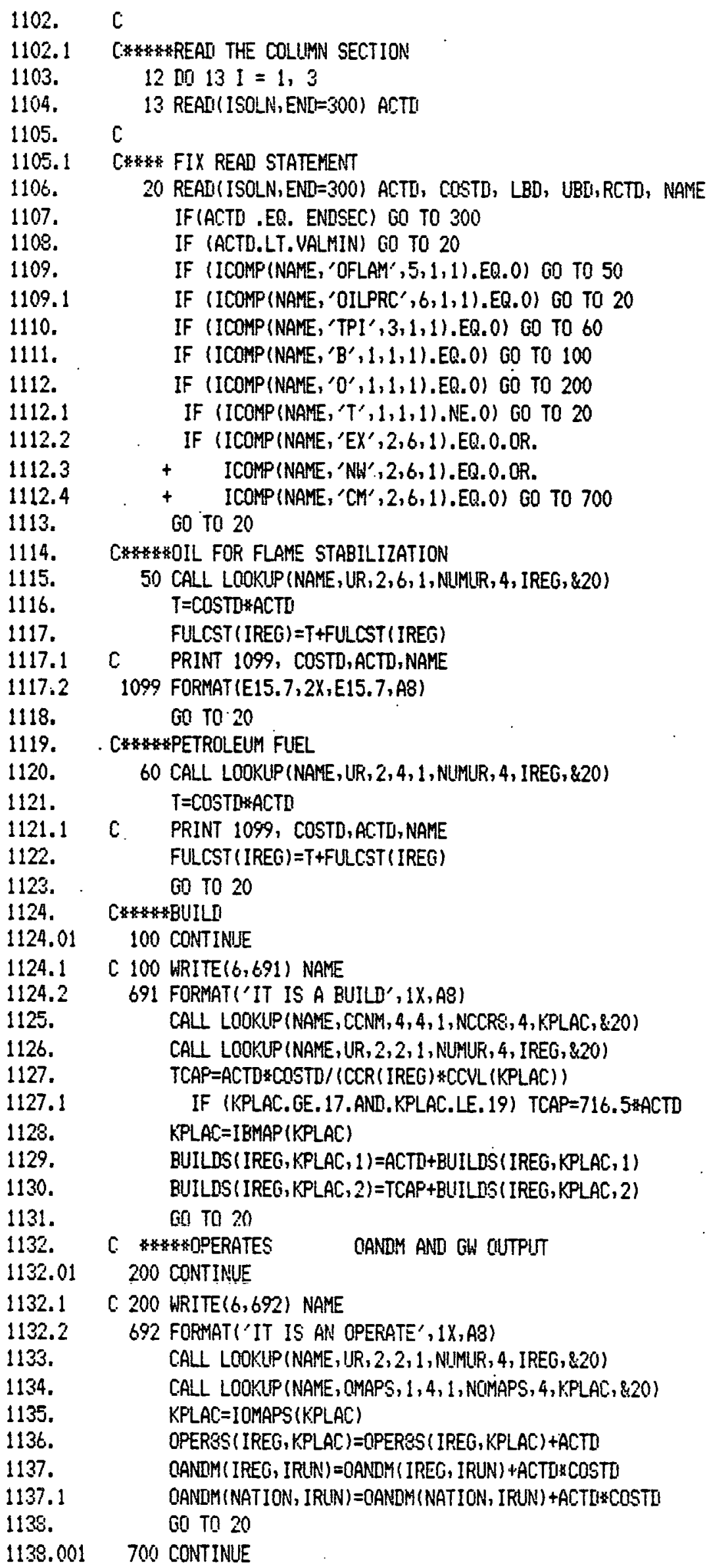


1138.01

1138.02

1138.03

1138.04

1138.041

1138.05

1138.06

1138.07

1138.08

1138.09

1138.1

1138.11

1138.111

1138.12

1138.121

113.13

1138.14

1138.15

1138.16

1139.161

1138.162

1138.17

1138.18

1138.19

1138.2

1138.201

1138.21

1138.22

1138.23

1138.24

1138.25

1138.26

1138.27

1141.

1142.

1143.

1144.

1145.

1146.

1147.

1148.

1149.

1150.

1150.1

1150.2

1151.

1152.

1153.

1154.

1154.11

1154.12

1154.13

$\$ 154.14$

1154.15

1154.16

1154.17
C* NEWH CODE-TRANGFERS AND TRANGMISSION BUILDS HERE- THIS CODE

C 700 WRITE $(6,591)$ NAME, ACTD, COSTD

591 FORMAT(' ITS TRANS', $2 X, A 8$, , ACTD = ',F15.',' COSTI = ',FI5.5)

C* LOOKS FOR CAPITAL EXPENDITURES FOR ALL LINES NOT JUST NEW $I N \mid=0$

CALL LOOKUP (NAME, UR, 2, 2,1, NUMUR, 4, IFR, 820 )

CALL LOOKUP (NAME, UR, 2, 4, , NUNINR, 4, ITO, \&20)

CALL LOOKLP (NAME, 'EX' $2,6,1,1,4$, IRA, \&6,6)

CALL LOOKUP (NAME, TLNKEX, 4,2,1, NTLEX, 4, ILINK, \&999)

TRANEF=TEFFEX (ILINK)

60 TO 697

696 CALL LOOKUP (NAME, TLNKNH, 4, 2, 1, NTLNW, 4, ILINK,

$I N W=1$

TRANEF=TEFFNW(ILINK)

697 CONTINUE

C 697 WRITE $(6,694)$ IRUN, IFR, ITO, ACTD, NAME

694 FORMATI' IRUN= ', 13,2X,'FROM REG $={ }^{\prime}, 13,2 X,{ }^{\prime}$ TO REG $=', 13,2 \mathrm{X}$,

$+{ }^{\prime}$ AMT $=', F 10.4,^{\prime}$ NAME $='$, A8)

CHARGE $=($ FUELP (ITO, IELEC) + FUELP (IFR, IELEC $)) / 2.0$

C. WRITE $(6,661)$ CHARGE, ACTD, TRANEF

661 FORMATl' CHARGE ',F20.4,' ACTD ',F20.4,' TRANEF ',F20.4)

AVEFUE (IFR, IRIN) =AVEFUE ( IFR, IRUN) -CHARGE *ACTD

AVEFUE (ITO, IRUN $)=$ AVEFUE (ITO, IRUN $)+$ CHARGE $* A C T D$

TRNSFR(IFR, IRUN)=TRNSFR(IFR, IRUN) -ACTD

TRNSFR (ITO, IRUN) =TRNSFR (ITO, IRUN) +ACTDKTRANEF

IF(INH.EQ.0) GO TO 20

TCAP=ACTD $*$ COSTD/CCR(IFR)

BUILDS(IFR, 13,1)=ACTD + BUILDS(IFR, 13,1)

EUJILIS $($ IFR, 13,2)=TCAP + BUILDS $($ IFR, 13,2)

GO TO 20

CH:

300 D0 $330 \quad I=1$, NUMUR

(.) KRE(G=NURCEN(I)

DO $305 \mathrm{~J}=1,14$

COPER $(I, J, I R I N)=\operatorname{COPER}(I, J, I R(N)+O P E R B S(I, d) * \operatorname{TEFF}(I)$

COPER(NATION, J, IRUN)=COPER(NATION,, IRUN)+OPERBS $(I, J)$ \&TEFF(I)

DO $306 \mathrm{~K}=1$, ?

CBUILD(NATION, $, K, K, I R U N)=$ CBUILDD(NATION, $J, K, I R U N)+$ BUILDS $(I, J, K)$

306. CBUILD $(I, J, K, I R U N)=$ CBUILD $(I, J, K, I R(N)+$ BUILDS $(I, J, K)$

305 CONTINUE

$\mathrm{T}=0$.

Do $310 \mathrm{~N}=1,18$

$T=F U E L P(I, J) \& F U E L Q(I, d)+T$

C WRITE $(t, 662) I, J, F U E L P(I, d), F U E L Q(I, J)$

662 FORMATl' I= ',I3, ' $J=^{\prime}, I 3$, ' PRC ',F20.4,' QTY ',F20.4)

310 CONTINUE

AVEFUE $(I, I R U N)=$ AVEFUE $(I, I R L N)+T+F U L C S T$ (I)

AVEFIE (NATION, IRUN) =AVEFUE(NATION, IRUN) +T+FLLCST(I)

330 CONTINUE

C

C * CALCULATE THE LOSSES WHICH OCCUR FROM

C. GENERATION TO SALES, SLMSS. (IE. TD LOSSES AND TRANGFERS)

C

SALESN $=0.0$

DO 615 JRES $=1$, NUMUR

GENER $=0.0$ 
1154.18

1154.19

1154.2

1154.21

1154.22

1154.23

1154.24

1154.25

1154.26

1154.27

1154.28

1154.29

1154.3

1154.31

1154.32

1154.33

1154.34

1154.35

1154.36

1155.

1155.1

1155.2

1155.3

1156.

1500.

1500.001

1500.002

1500.003

1500.004

1500.005

1500.006

1500.007

1500.008

1500.01

1500.02

1500.03

1500.04

1500.05

1500.06

1500.07

1500.08

1500.09

1500.111

1500.112

1500.113

1500.114

1500.115

$1500.116 \quad \mathrm{C}$

$1500.117 \mathrm{C}$

$1500.118 \quad \mathrm{C}$

1500.119

1500.12

1500.13

1500.14

1501.

1502.

C

c
DO 614 NFLANT $=1,14$

614 GENER=GENER+COPER (JREG, NPLANT, IRUN)/TEFF (JREG)

SALES $=$ TEFF (JREG) \& (GENER+TRNSFR (JRES, IRUN) )

SLOSS (JREC;, IRLNU) =SALES/GENER

AIUGEN=GENER+TFNGFR (JREG, IRURS)

PRINT 1008, JREG, ADJGEN

1008 FORMATY' CALCILATED ADUIISTEN GENERATION FOR REGION ', $12,2^{\prime}$, F15.4, SALESN=SALESN+SALES

615 CONTINLE

GENER $8=0.0$

DO 620 NPLANT $=1,14$

620 GENER8=GENER8+COPER(NATION, NPLANT, IRUN)/TEFF (JREG)

SLOSS (NATION, IRUN) = SALESN/GENER8

DO 625 \REG $=1$, NATION

625 SLOSS ( JREG, IRIN) =1. O-SLOSS (JREG, IRUN)

IO 791 JREG $=1$, NATION

C. PRINT 1011, JREG, TRNGFR(JREG, IRUN)

1011 FORMATI' NET TRANGFERS FOR REGION ',12,'IS',':',F10,4)

791 CONTINUE

RETURN

999 WRITE $(6,998)$

998 FORMAT!' LOOKUP ERROR IN EXTRAC')

STOP

END

SUBROUTINE COMPUT

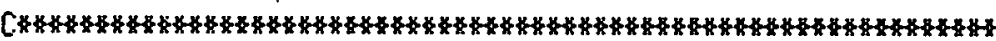

C

C.

INSERT GMG COMMON HERE

$\mathrm{C}$

C

C

REAL $* 4$ LOAD, LOADF

COMMON/URIATA/NUMUR, NPLTP, PLTYPE(50), PLID(50), NUMUE, UE (24), 1NOLNDS, BUE (13), UR (44), NDYDTP, DMDNAM (7), LOADI (4), LOADF $(44,4)$, 2CAPF $(44,4,4)$, NTLEX, TLNKEX (99), TPRCEX (99), TEFFEX (99), NTLNW, XTLNKNW (99), TPRCNW(99), TEFFNW(99), TEFF (44), TPRCE (44), OGCAP (44), XBLEND $(13,24)$, FUEL JE $(44,24)$, NPLID, RHSCL $(44), \operatorname{RHSC} 9(44), \operatorname{PENL}(3,4)$

7, NUBLND, UBLEND $(31,5)$

C C (1) .

END GME COMMON

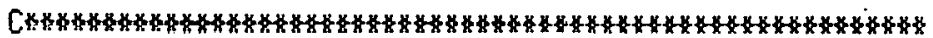

C

COMMON/SHARE/ FUELP $(44,23)$, FUELQ $(44,23)$,

$X$ EUIILIS $(44,14,2)$, OPER8S $(44,14)$

COMMON /REG/CBUILD $(45,14,2,3)$, COPER $(45,14,3)$, AVEFLE $(45,3)$

$x$, HEL 75 (45), OANDM $(45,4)$, NUCFUL (45) 


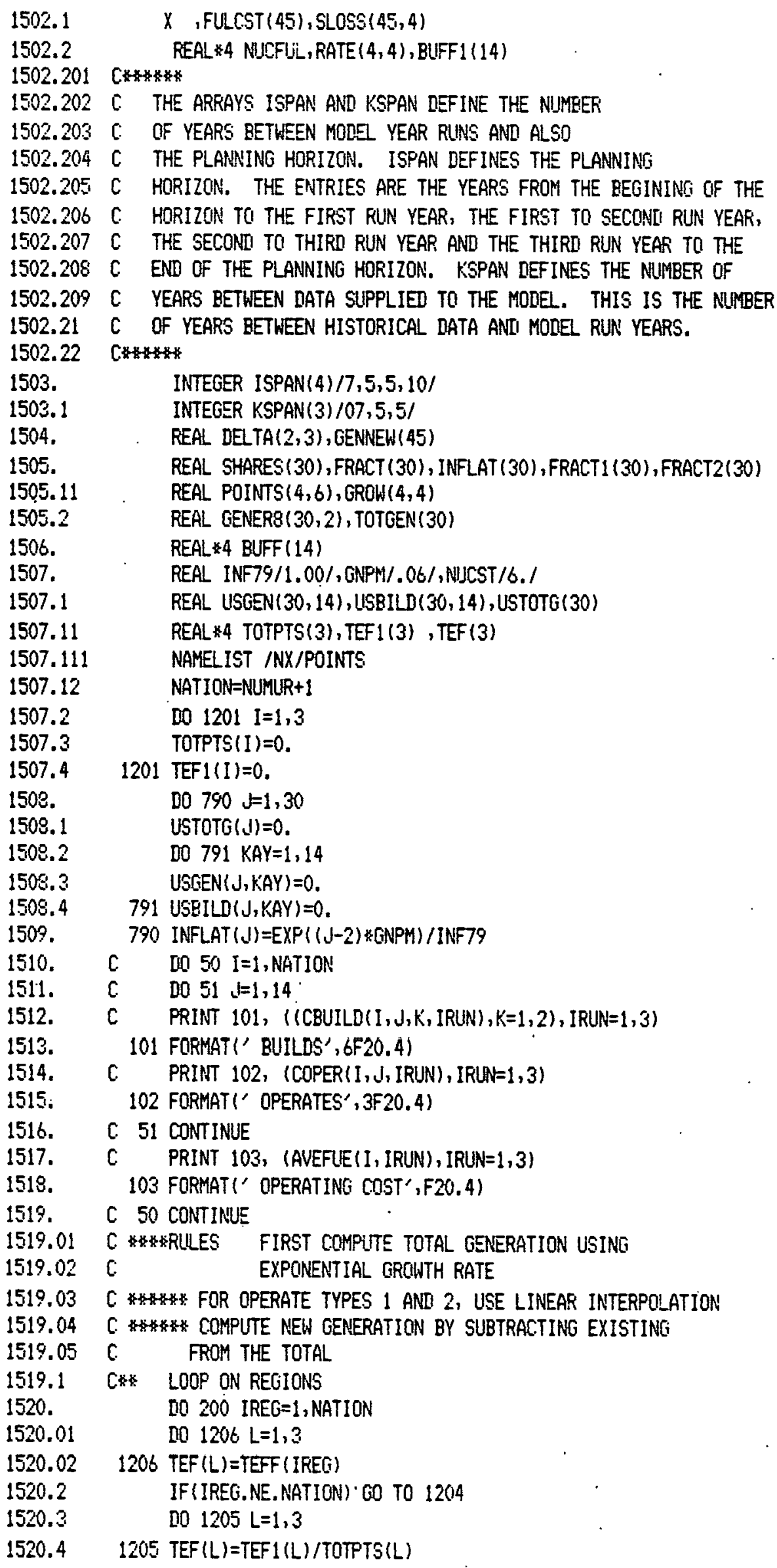




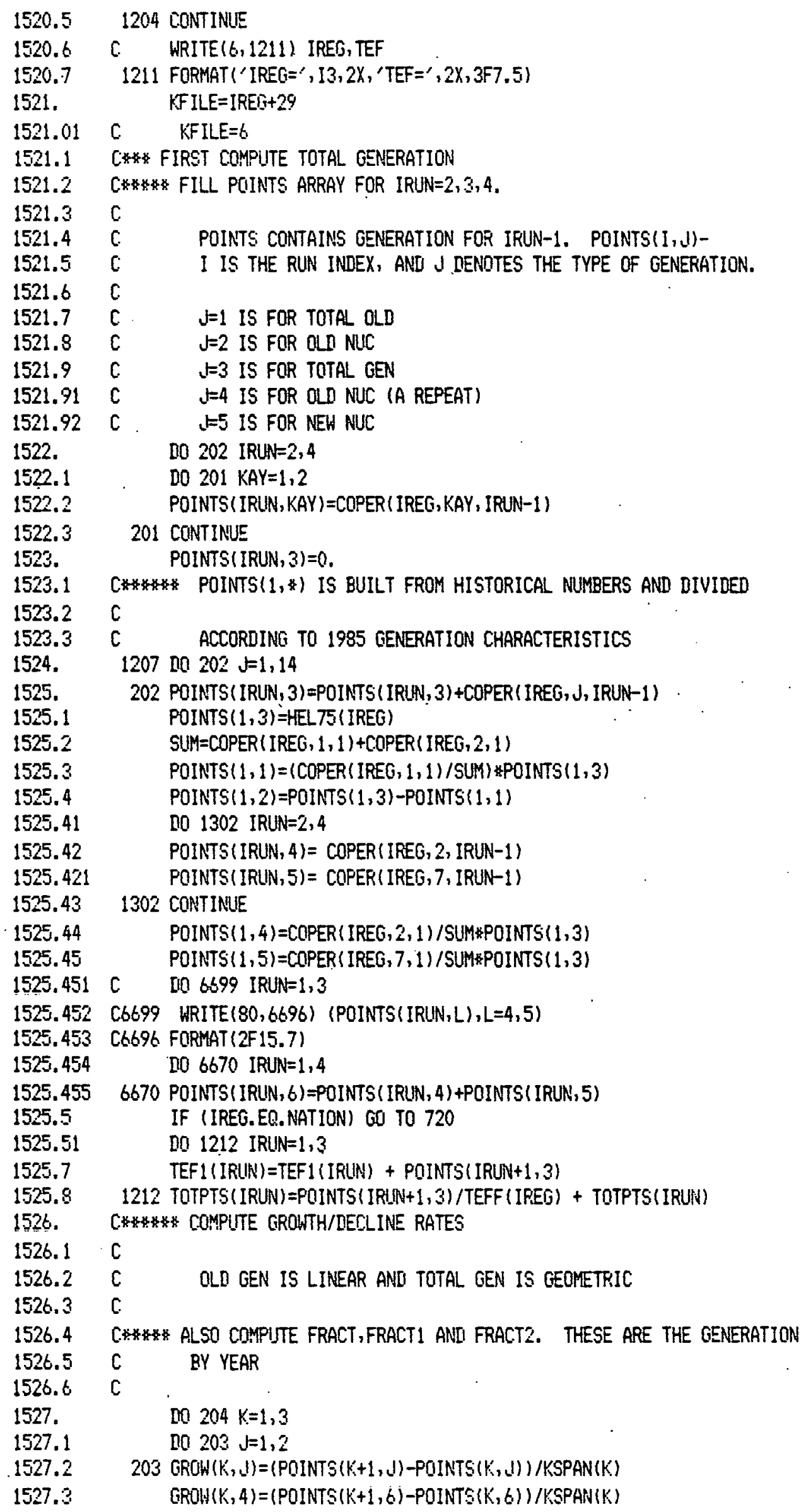


1528. 204 GROH $(K, 3)=A L O G(P O I N T S(K+1,3) / P O I N T S(K, 3)) / K S P A N(K)$

$1528.1 \quad$ C. DO 6693 IRA $=1,4$

1528.2 C6693 WRITE(90,6692) (POINTS(IRA, IL), IL=1,3)

1528.3 6692 FORMAT I 4F 15.7)

1529. $J A Y=1$

1530. $\quad K A Y=1$

1531. In 205 IRUN $=1,3$

1532. SLM $=0$.

1533. IYR=ISPAN(IRUN)

1533.1 IYR=KSPAN (IRUN) - ISPAN (IRLN)

1533.2 IBEGYR=JAY

1534.

1534.1

DO $206 \mathrm{KYR}=1$, IYK

SUM $2=0$.

1534.11

1534.12

1534.2

$J Y R=J Y R+1$

1534.21

1534.3

1536.

1536.001

1536.002

1536.01

1536.02

1536.1

1537.

.1537 .1

1538.

1539.

$J A Y=J A Y+1$

DO $208 \mathrm{~J}=1,2$

GENERS $(J A Y, J)=P O I N T S($ IRUN, J)+GROW (IRUN, J)*JYR

208 SUM2 $=S U M 2+$ GENERB $(J A Y, J)$

FRACT ( JAY) =PDINTS (IRUN, 3) *EXP (GROM (IRUN, 3)*JYR)-SUM2

FRACT 1 ( JAY $)=($ FRACT ( JAY $)+$ SUA2) /TEFF (IREG)

FRACT2 (JAY) $=$ (POINTS (IRLN, 6) + GROW (IRUN, 4) $*$ JYR) /TEFF (IREG)

TOTGEN $(J A Y)=$ FRACT $($ JAY $)+S U M 2$

USTOTG ( JAY) = USTOTG (JAY) + TOTGEN (JAY)

IF (FRACT (JAY).LE. O.) FRACT (JAY) $=0$.

206 SUM $=$ SUM+FRACT (JAY)

GENREW(1)=FRACT ( JAY) +RACT (IBEGYR)

DO $207 \mathrm{KYR}=1$, IYR

$K A Y=K A Y+1$

1540. 207 SHARES $(K A Y)=$ FRACT $\left(K^{\prime} A Y\right) /$ SUM

1541. 205 CONTINUE

1542. C PRINT 701, (SHARES (JAY), JAY $=2,18$ )

1543. 701 FORMAT!' SHARES', 18F5.2)

1544. C PRINT 702, (FRACT (JAY), JAY $=2,18$ )

1545. 702 FORNAT(' FRACTION", 18F5.0)

1546. 720 DO $210 \mathrm{~J}=1,14$

1547.

1548.

1549.

1550.

IF (CBUILD(IREG, $, 1,1,3), L E .0,001)$ GO TO 210

[II $213 \mathrm{KAY}=1,2$

DELTA $(K A Y, 1)=$ CBUILD (IREG, J,KAY, 1)

I0 $211 \mathrm{~K}=2,3$

1551.

DEL TA $(K A Y, K)=C B U I L D(I R E G, J, K A Y, K)-C B U I L D(I R E G, J, K A Y, K-1)$

1552.

1553.

211 CONTINUE

213 CONTINUE

JAY $=1$

1555.

[IO 212 IRUN $=1,3$

1557. IYR=ISPAN (IRUN)

1558.

$155 \%$.

1560.

1561 .

1562.

1562.1

1562.2

1562.3

1563.

1563.1

IF (DELTA11,IFUN).LT.0.001) G0 TO 712

AVEC $=D E L T A(2$, IRUN $) / D E L T A(1$, IFUN)

712 DO $214 L=1$, IYR

$J A Y=J A Y+1$

IF (DELTA(1, IRUN).LT.0.001) G0 T0 214

IF (IREG.NE. NATION) GO TO 721

$Q=U S B] L D(J A Y, J)$

GO TO 722

$7210=$ SHARES (JAY) *DEL TA $(1$, IRUN $) * 1000$

USBILD ( JAY, J) = USBILD $(J A Y, J)+Q$

1564.

722 BUFF(1)=AVEC $/$ INF79 
1565. WRITE(KFILE, 105) JAY, d, (1 , BUFF(1)

1566. 105 FORMAT (2I5,F20.3, F20.3)

1567. 214 CONTINIE

1568.

1568.01

1568.02

1568.03

1568.04

1588.05

1568.06

1568.07

1568.08

1568.09

1568.1

1568.11

1568.12

1568.13

1568.14

1568.15

1568.16

1568.17

1568.18

1568.19

1568.2

.1568 .21

1568.22

1568.23

1568.24

1568.25

1568.26

1568.27

1568.28

1568.29

1568.3

$156 \%$.

1569.01

1569.02

$156 \% .03$

1569.04

1569.05

1569.06

1569.07

1573.

1574.

1574.1

1574.5

1574.6

1576.

212 CONTIMUE

C. CK $*$ NOW EXTRAPOLATE BUILDS TO YEAR 2005

CHEFIRST EXTRAFOLATE SHARES TO YEAR 2005, ALSO CALCULATE FACTUR. CK $\$$ FACTOR IS THE RATIO OF NEW GEN FROM 95-2005 TO THAT OF 90-95. C* TOTAL GEN IS ASSUMED TO GROW AT SAME RATE AS IT IIII IN 90-95

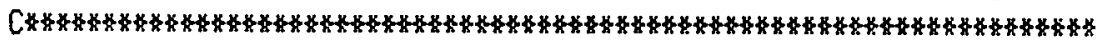
IRUN $=4$

JAYI $=$ JAY

IYR=ISPAN (IRUN)

LO $300 \mathrm{JI}=1, \mathrm{IYR}$

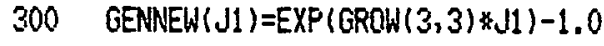

AOLD $=0.0$

Do $310 \mathrm{Jl}=1$, IYR

$J A Y=J A Y+1$

SHARES (JAY) $=$ (GENNEW (J1)-ADLD) /GENNEW ( IYR)

AOLLIVGENNEW(J1)

310 CONTINUE

FACTOR=GENAEW(IYR) *TOTGEN(JAYI)/(POINTS (4,3)-POINTS (3,3))

IF (DELTA 1,3$)$.LT. .001) GO TO 210

DO $320 \mathrm{Jl}=1$, IYR

JAYI $=J A Y 1+1$

IF (IREG .NE. NATION) GO TO 321

Q:USBILDI JAYY, J)

60 TO 322

$3210=$ FACTOR $*$ DEL TA $(1,3) *$ SHARES $(J A Y 1) * 1000.0$

USBILI ( LAY1, J) $=$ USBILD ( JAY1, J) +Q

322 WRITE(KFILE, 105) JAY1, J, I, BUFF(1)

C 322 CONTINIE

320 CONTINUE

210 CONTINUE

$J A Y=0$

$\operatorname{BUFF}(1)=0$.

WRITE(KFILE, 105) - JAY, JAY, BUFF (1), BUFF(1)

C*

C. NOW FINER CATEGORIES OF NEW GENERATION ARE INTERPOLATED BY YEAR.

C. * * *UMENI IS TOTAL GENERATION BY NEW PLANTS AT END OF PERIOU

C *SUMBEG IS TOTAL GENERATION BY NEW PLANTS AT BEGINNING OF PERIOD

C $\triangle A Y=1$

DO 220 IRUN $=1,3$

SUM $=0$.

D0 $226 \quad \mathrm{~J}=3,14$

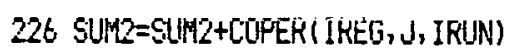

KYR=ISPAN (IRLN)

1577.

1578.

1578.1

1578.2

1578.3

1578.4

$157 \%$.

DO 225 IYK $=1, k$ VR

$J A Y=J A Y+1$

IF (IREG. NE. NATION) GO TO 224

II0 $223, \leqslant 1,2$

223 $\operatorname{BUFF}(\mathrm{U})=\bigcup \operatorname{USGEN}($ JAY, J)

GO TO 229

1579.1

C 224 DUIFF (1) =GENERB $($ JAY , 1)+GENERB $($ JAY , 2)

224 CONTINUE 


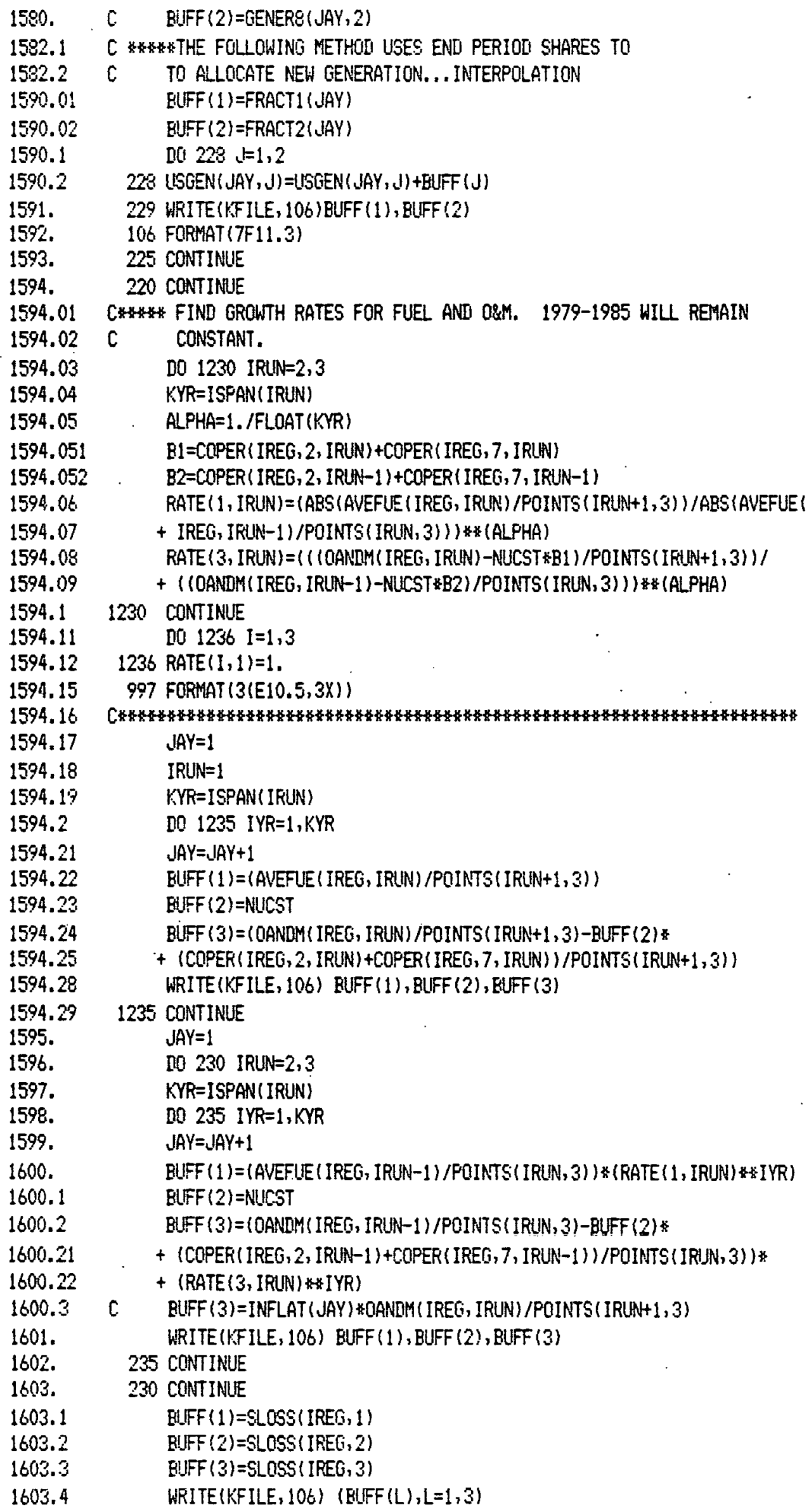




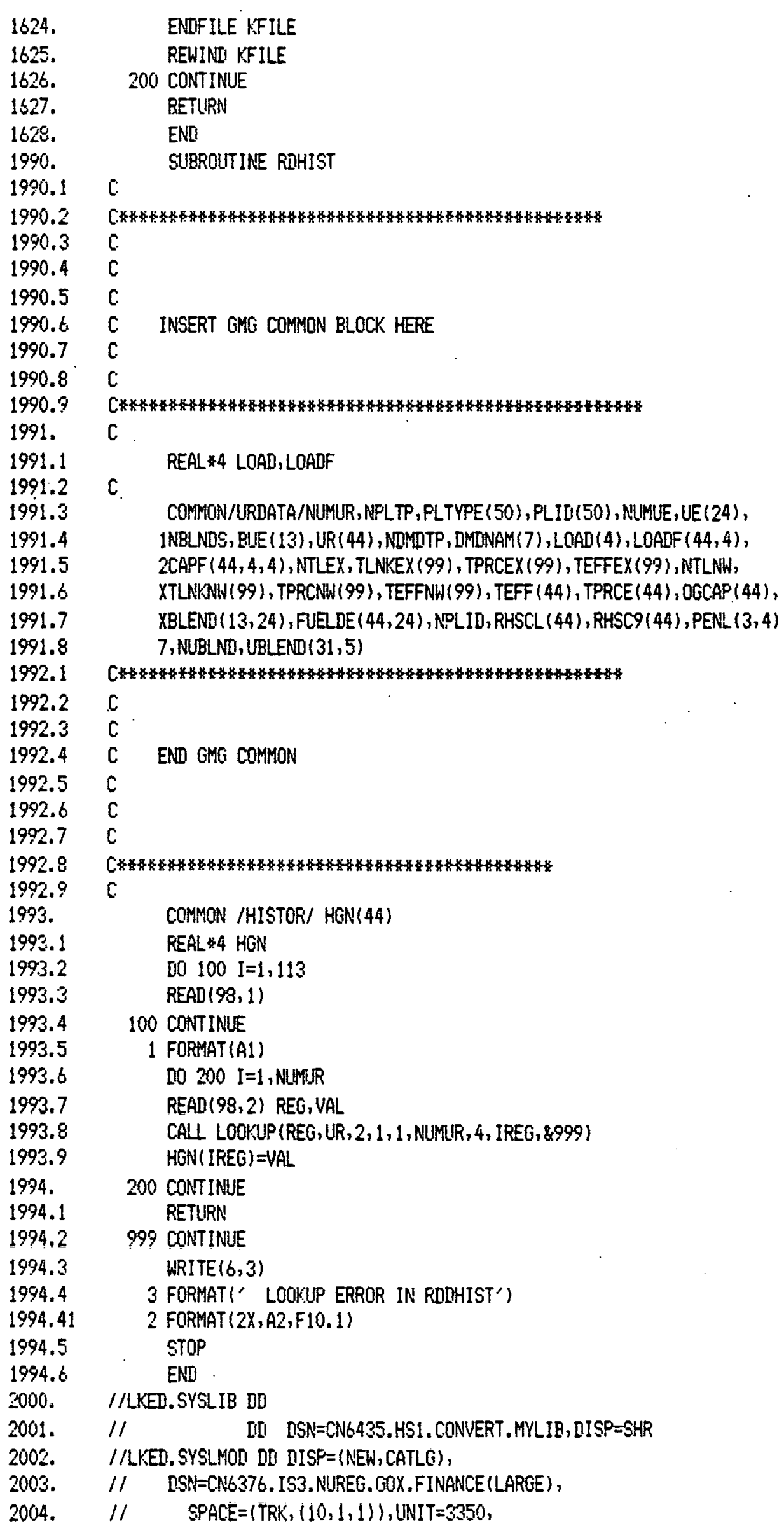


$-18-$

2005. $/ 1 \quad$ DCE $=$ (DSORG $=P Q$, RECFM=U, BLKSIZE=7294) 


\subsection{THE F INANCIAL SENSITIVITY PROGRAM (FSP)}

The purpose of FSP is to estimate the sensitivity of the financial parameters to changes in capital additions. These derivatives are calculated for each of the NCM build years 1985, 1990, and 1995. FSP.begins by successively running NUFS, each time varying the level of capital additions. Next, it examines the results and forms partial derivatives by dividing the change in the value of the parameters by the percentage change in the level of capital additions.

FSP is a set of six job steps. The first job step creates four different capacity expansion forecast files for NUFS. The first file is the one produced by the NCM/NUFS interface. The second file is formed by lowering the costs of all capacity additions in the years 1979-1985 by a given percentage (the value of the percentage is a user input). similarly, the third and fourth files are formed by lowering costs of builds for the years 1986-90, and 1991-1995, respectively. Each of the latter three files correspond to the original NCM solution's capacity additions. lowered in the solution years 1985, 1990, and 1995. It should be noted that the extrapolated builds for the years past 1995 are also lowered in the fourth file.

The next four job steps each run NUFS using the different capacity additions files created in step 1. Each of the steps produces a forecast of the financial parameters corresponding to different capacity addition files. Each of these sets of forecasts is passed to the final job step.

The final step forms the derivatives as follows. For the 1985 derivatives, the forecasts from NUFS for the 1985 reduced capacity case are subtracted from the forecasts from NUFS for the original NCM solution. These differences are then divided by the percentage of capacity additions cut in the years 1979-1985. This yields the derivatives for the 1985 capital additions. The 1990 and 1995 derivatives are calculated in a similar manner. These derivatives, combined with other data, are then written to a file which will be used by the CAAP.

A flow of data diagram for FSP is shown in Figure 2. A listing of FSP now follows. 
FIGURE 2

DATA FLOW FOR FSP

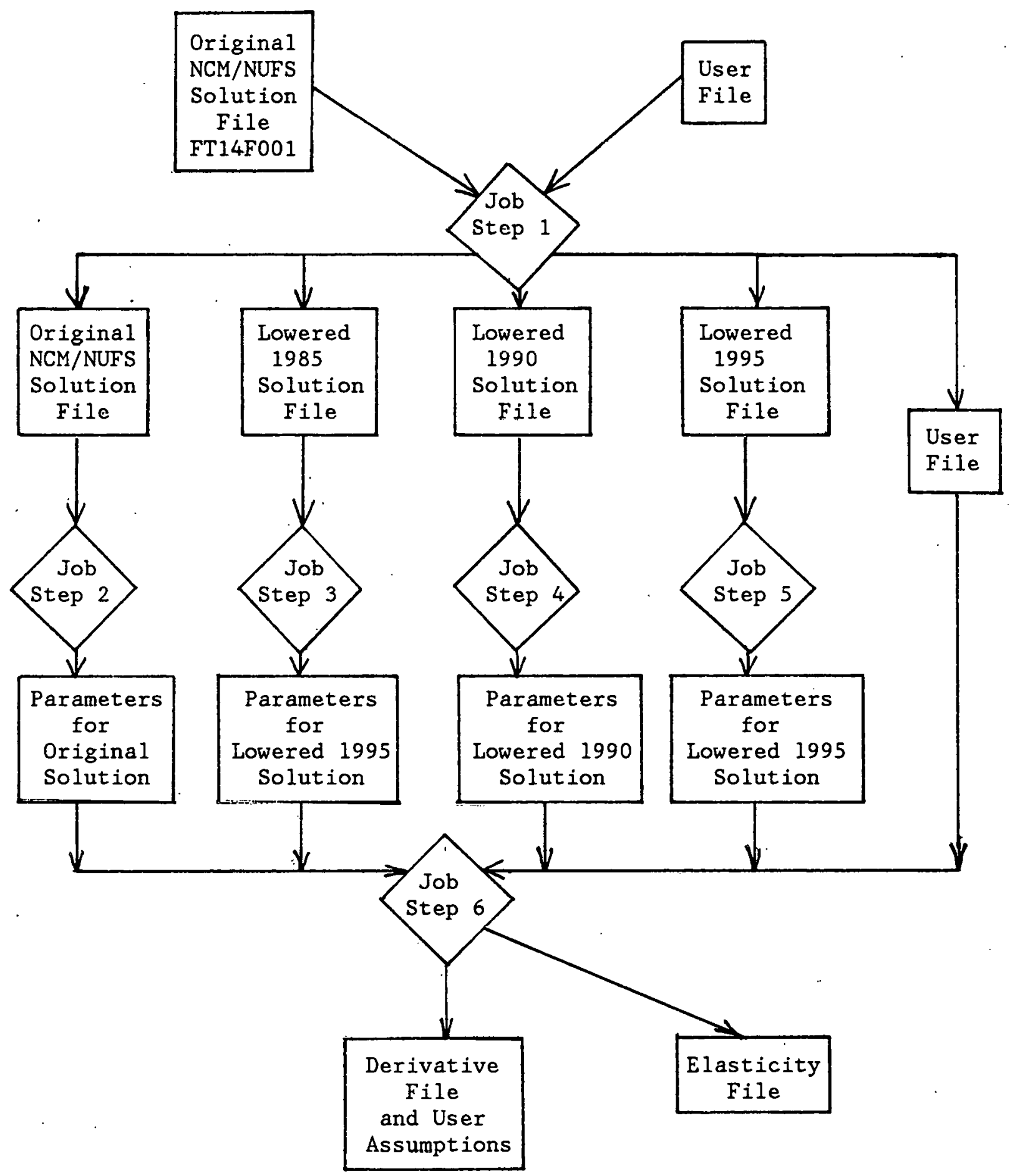


1. //MI2LNUFS JOB $(6376,002,, 15),{ }^{\prime}$ COMPILE', TIME $=(0,19)$

2. I/STEP1 EXEC FORTGCG

3. I/FORT.SYSIN OI *

4. C. NLREG.FSP FINANCIAL SENGITIVITY PROGRAM

5. C. HRITTEN BY MARK INGLIS ICF INC. OCTOEER 29,1981

6. C. THIS MOIULE IS DESIGNED FOR ANALYSIS OF ELECTRIC UTILIT: REGIONS WHICH

7. C. ARE DETERMINEII TO HAVE NON-FINANCIALLY FEASAELE CAPACITY EXPANSION PLANS.

8. $C$

9. C THIS FIRST STEP IS DESIGNED TO CREATE INTERFACE FILES FOR NUFS WHICH

10. C HAVE CAPITAL COSTS LOWERED IN GIVEN YEARS BY A GIVEN PERCENTAGE. THESE

11. C FILES WILL BE USED IN COMBINATION WITH NUFS TO DETERMINE SENSITIVITY

12. C OF FINANCIAL PARAMETERS TO CHANGES IN CAPITAL ADDITION COSTS.

13. $\mathrm{C}$

14. DIMENSION ISPAN(5), DELCAP(5), TEMP (20), TARGET(4),

15. 1 TOTCAP(5), WGTPEN $(4,25)$

16. DATA DELCAP $/ 5 * 0.01$, TOTCAP/5*0.01, ISPAN $/ 2,9,14,29,0 /$,

17. * NYRS3, NOSPAN/18,3/

18. C*VARIABLE DEFINITIONG:

19. C NOSPAN- NMMER OF SOLUTIONS IN OPTIMIZATION (3 FOR MEFS, NCM)

20. C ISPAN- YEARS DEFINING EACH SOLUTION

21. C NYRS3- INTEGER REPRESENTATION FOR 1995

22. C*\#FIRST, READ IN INPUTS WHICH DEFINE THE SCOPE OF RUN:

23. C NREG- NUMBER OF INFEASIBLE REGIOAS

24. C NAME-TITLE OF REGION

25. C RATE- PERCENTAGE REDUCTION TO BE MADE IN CAPITAL COSTS

26. C NYRS1, NYRS2- RANGE OF YEARS OF HHICH TO LOOK AT FINANCIAL PARAMETERS

27. C TARGET- TARGET VALUES FOR FINANCIAL PARAMETERS

28. C $\ldots$

? L 78/L

78. C*NOW WRITE OUT TEMPORARY FILE TO BE PASSED.

79. WRITE $(20,5)$ NAME

80. WRITE $(20,210)$ RATE

81. WRITE $(20,6)$ NYRS1, NYRS2

82. WRITE $(20,210)$ TARGET

83. $D 0220 \mathrm{I}=1,4$

84. 220 WRITE $(20,210)$ (WGTPEN $(1, J),,=1$, NVRS)

85. 210 FORMAT(7 (E10.4, $1 X))$

86. 6 FORMAT (2I4)

87. WRITE $(20,6)$ NOSPAN

88. WRITE(20, 210) (DELCAP(I), I=1, NOSPAN), (TOTCAP(I), I=1, NOSPAN)

89. 300 CONTINUE

$\ldots$

$?\llcorner 100$

100. $15 * 100.0$ PENALTY WEIGTS FOR AFLDC PERCENTAGE OF INCOME

? L 101/L

101. $15 \% 100.0$ PENALTY WEIGHTS FOR ACTUAL RETURN ON ERUITY

102. $15 \% 100.0$ PENALTY WEIGHTS FOR EXTERNAML FINANCING PERCENTAGE

103. UM

104. .20 PERCENTAGE REDUCTION IN BUILDS

105. $\ldots$

? M 103

103. UM

ALTERS ? RUA

103. VA THITL OF GECOND INFEASIBLE REGION

ALTERS ? 
1. //MI2UNUFS JOE $(6.376,102,, 15),{ }^{\prime}$ COMPILE', TIME $=(0,19)$

2. I/STEP1 EXEC. FORTGCG

3. I/FORT. SYSIN DD*

4. C. NLREG. FSF FINANCIAL SENSITIVITY PROGRAM

5. C. WRITTEN BY MARK INCLIS ICF INC. OCTOBER 29,1981

6. C. THIS MODULE IS DESIGNED FOR ANALYSIS OF EIECTRIC ITILITY RESIONS WHICH

7. C ARE IETERMINED TO HAVE NON-FINANCIALLY FEASABLE CAPACITY EXPANSION PLANS.

8. $C$

9. C THIS FIRST STEP IS DESIGNED TO CREATE INTERFACE FILES FOR NUFS WHICH

10. C. HAVE CAPITAL COSTS LOWERED IN GIVEN YEARS BY A GIVEN PERCENTAGE. THESE

11. C FILES WILL BE LISED IN COMBINATION WITH NUFS TO DETERMINE SENSITIVITY

12. C OF FINANCIAL PARAMETERS TO CHANGES IN CAPITAL ADDITION COSTS.

13. $C$

14. DIMENSION ISPAN(5), DELCAP (5), TEMP (20), TARGET(4),

15. 1 TOTCAP (5), WGTPEN $(4,25)$

16. DATA DELCAP $/ 5 * 0.0 /$, TOTCAP $/ 5 * 0.0 /, 1$ SPAN $/ 2,9,14,29,0 /$,

17. $\quad$ NYRS3, NOSPAN/18,3/

18. C* *VARIABLE DEFINITIONS:

19. C NOSPAN- NUMBER OF SOLUTIONS IN OPTIMIZATION (3 FOR MEFS, NCM)

20. C ISPAN- YEARS DEFINING EACH SOLUTION

21. C NYRS3- INTEGER REPRESENTATION FOR 1995

22. C* \#FIRST, READ IN INPUTS WHICH DEFINE THE SCOPE OF FUN:

23. C NREG- NUMBER OF INFEASIBLE REGIONS

24. C NAME- TITLE OF REGION

25. C RATE- PERCENTAGE REDUCTION TO BE MADE IN CAPITAL COSTS

26. C NYRS1, NYRS2- RANGE OF YEARS OF WHICH TO LOOK AT FINANCIAL PARAMETERS

27. C TARGET- TARGET VALLES FOR FINANCIAL PARAMETERS

28. C WGTPEN- PENALTY WEIGHTS FOR NOT SATISFYING CONSTRAINTS

29. READ $(8, *)$ NREG

30. WRITE $(20,6)$ NRES

31. DO $300 \mathrm{~K}=1$, NREG

32. READ (8,5) NAME

33. 5 FORMAT(A2)

34. READ (8, *) RATE

35. READ(3, *) NYRS1, NYRS2

36. NYRS=NYFS2-NYRSI1+1

37. READ(\&, $*$ ) TARGET

38. DO $15 \quad I=1,4$

39. 15 READ (8, *) (WGTPEN $(1, J), J=1$, NYRS)

40. CWNOW THE BUILDS SECTION OF THE NUFS INTERFACE FILE IS READ, ALTERED,

41. C**AND PRINTED CUIT TO THE NEN INTERFACE FILES.

42. 100 READ $(10, *)$ IYR, JPLANT, PLTCAP, CAPCST

43. WRITE(14,30) JNR, JPLANT, PLTCAP, CAPCST

44. CAPRED $=$ CAPCST $*(1,0-$ RATE)

45. C* * NOH WRITE OUT BUILD TO EACH OF FOUR FILES:

46. C* FILE $11=$ NCM 85 BUILDS REDUCED

47. C FILE $12=$ NCM 90 QUILDS REDUCED

48. C FILE $13=$ NCM 95 PLUS EXTRAPALATIONS BUILIS REIUCED

49. C* FILE 14 = BASE NCM SOLUTION

50. C*** FILE $15=$ ALL EUILDS REDUCEII

51. [0 10$]=1$, NOSPAN

52. IFILE $=I+10$

53. IF ((JYR.GE. ISPAN(I)) . AND. (JIYR.LT.ISFAN(I¥1))) GO TO 20

54. C\&\&THE CAPCST IS NOT TO BE REDUCED FOR THIS FILE (IFILE)

55. HRITE(IFILE, 30) JYR, JPLANT, PLTCAP, CAPCST 


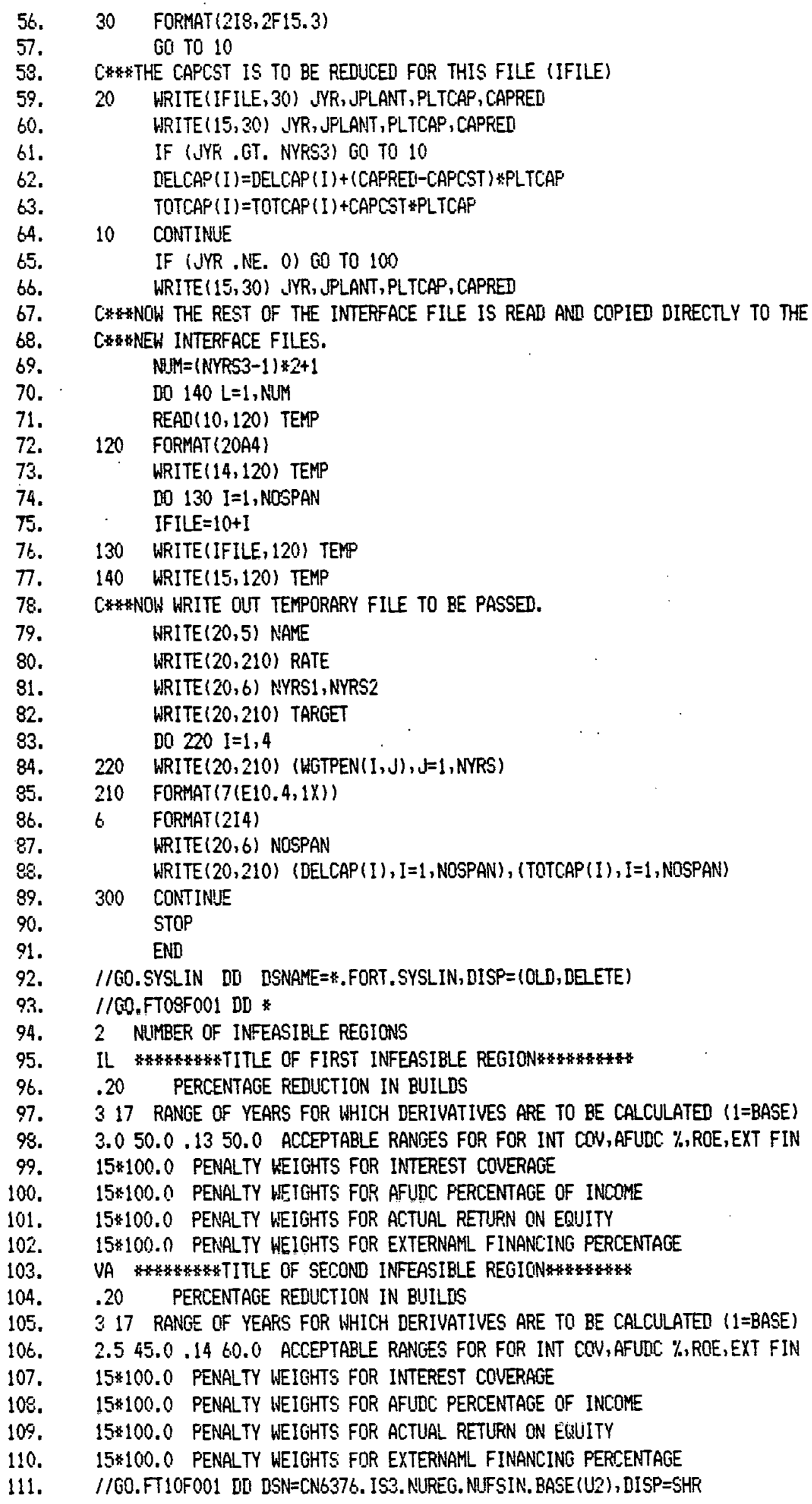


112. $/ / \quad$ DD DSN=CNE376. IS3. NUREG. NLIFSIN. BASE(U1), DISP=SHR

113. $/ / 60 . F T 11 F 001$ DD DISP $=(N E W, P A S S)$, DSN $=\& \&$ TEMPBS, $S P A C E=(T R K,(1,1))$,

114. I/ DCB=(RECFM=FB, LRECL $=80$, BLKSIZE $=6160, D S O R G=P S)$, LNIT=DASD

115. $/ /$ GO.FT12F001 DI DISP $=($ (NEW, PASS), DSN=\&\&TEMP90, SFACE $=(T R K,(1,1))$,

116. $/ 1 \quad$ DCE=(RESFP=FB, LRECL $=30$, BLKSIZE $=6160, D S Q R G=P S)$, UNIT=DASD

117. //GO.FT13F001 DD DISP=(NEW, FASS), USN=\&\&TEMPOS, SFACE $=(T R K,(1,1))$,

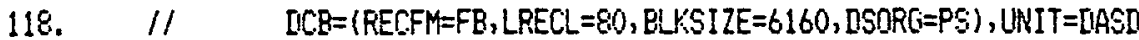

119. $/ /$ G0. FT14FO01 DII DISP =(NEH, PASS), DSN=\&\&TEMPBS, SPACE $=(T R K,(1,1))$,

120. $/ 1 \quad$ ICB $=$ (RECFM $=F B, L R E C L=80, B L K S I Z E=6160, D S O R G=P S)$, LNIT $=D A S D$

121. //G0.FT15FO01 DI DISF $=$ (NEW, PASS), DSN=\&\&TEMPALL, SPACE $=(T R K,(1,1))$,

122. $/ 1 \quad$ UCB $=(R E C F M=F E, L R E C L=80, B L K S I Z E=6160, D S O R G=P S), U N I T=D A S I$

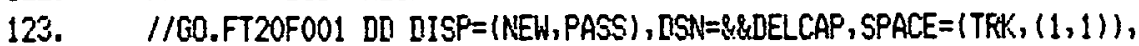

124. $/ 1 \quad I C B=(R E C F M=F B, L R E C L=80$, BLKSIZE $=6160, D S O R G=P S)$, UNIT $=D A S D$

125. $1 / 26$

126. // NOH, NUFS IS RUN 4 TIMES. THE FINANCIAL PARAMETERS FROM THE RUNS

127. //*UILL BE USED TO DETERMINE THE SENSITIVITY OF THE PARAMETERS TO

128. //*CHANGES IN CAPITAL COSTS. THIS INFORMATION IS USED TO CALCULATE

129. I/ENECESSARY CAPITAL CONSTRAINT FOR NCM OR MEFS.

130. $/ / 2 \%$ H

131. I/STEPPUN 1 EXEC PGM=TEST, REGION=700K

132. //STEPLIE DD DSN=CNE376. MI2. NUFS. LOAD, DISF $=$ SHR

133. I/FTO4FOO1 DD DUMMY

134. I/FTOGFOO1 DD DUMMY

135. I/FTO7F001 DD DUMMY

136. //FT08F001 DD DISP=(NEN,PASS), DSN=\&\&TEMPBSSR, SPACE $=(T R K,(1,1))$,

137. I/ DCB $=$ (RECFM $=F B, L R E C L=80$, BLKSIIE $=6160$, DSORC $=P S)$, LNIT $=D A S D$

138. I/FTO9F001 DD *

139. $*$ TITLE OF RUN

140. NUREG METH TEST $6 / 8$

141. * (1) NUMBER OF YRS; (2) NUMBER OF REGIONS; (3)OPT7: (4)OPT8

142. 17251

143. //FT1OFOO1 DD DSN=CNG376. MI2. NUREG. PRIVATE(IL), DISP=SHR

144. $1 /$ DD DSN=CN6376. MI2. NUREG. PRIVATE(VA), DISP=SHR

145. I/FTIIFOO1 DO DSN=CNG376.MI2. NUREG. PUBLIC(IL), DISP $=$ SHF

146. $/ / \quad$ DD ISN $=C N 6376$. MI2. NUREG. FUBLIC(VA), DISP $=$ SHR

147. I/FT12F001 DO IISHECNE376.MI2.NUREG.FORM1.FDS78(IL), DISP=SHR

148. $1 / \quad$ DD DSN=CN6376. MI2. NUREG. F(RM1. PDS78(VA), DISP $=S H R$

149. //FT13FOO1 DD DSN=CN6376. MI2. NUREG. FORM1M. PDS78 (IL), DISP=SHR

150. $/ / \quad$ DD DSN=CN6376. MI2. NUREG. FOOPMIM. PDS78(VA), DISFH=SHR

151. I/FT14F001 DD DSN=\&\&TEMPBS, DISP=(OLD, DELETE)

152. $/ /$ *

153. //STEPRUN2 EXEC PGM=TEST, REGION=700K

154. //STEPLIB DD DSN=CN6376. MI2. NUFS. LOAD, DISP=SHR

155. //FTO4F001 DD DAMMY

15i. I/FTOKFOOI DO DUMMY

157. I/FTOTFOOI DO DUMMY

158. //FTOBFOO1 DD DISP $=(N E W, P A S S)$, DSN=\&\&TEMPSSSR, SPACE $=(T R K,(1,1))$,

15\%. I/ DCQ $=$ (RECFM $=F B, L R E C L=80, E L K S I Z E=6160, D S O R G=P S), U N I T=D A S I$

160. I/FTOFFOOI DD *

161. * TITLE OF RUN

162. MUREG METH TEST 6/8

163. * (1)NUMBER OF YRS; (2) NUMBEF OF REGIONS; (3)OPT7; (4)OPTO

164. 17251

165. //FT1OF001 DD DSN=CN6376. MI2. NUREG. PRIVATE(IL), DISP=SHR

166. $/ 1 \quad$ DD DSN=CN6376. MI2. NUREG. PRIVATE (VA), DISP=SHR

167. //FTIIFO01 DO DSN=CNE376. MI2. NUREG. PUBLIC(IL), DISP $=S H R$ 
168. $/ / \quad D D D S N=C N 6376$. MI2. NUREG. PUBLIC(VA), DISF $=$ SHR

169. //FT12F001 DD DSN=CNE376. MI2. NUREG. FORM1. PDS78(IL), DISP =SHR

170. $/ 1 \quad$ UD DSN=CN6376. MI2. NUREG. FORM11. PDS78(VA), DISP=SHR

171. //FT13F001 DD DSN=CN6376. MI2. NUREG. FORMIM. PDS78(IL), DISP=SHR

172. I/ DII DSN=CNG376. MI2. NUREG. FORMIM. PDS78(VA), DISF =SHR

173. I/FT14F001 DII DSN=\&\&TEMP85, DISP=(OLD, DELETE)

174. $/ 1 \%$

175. //STEPRUNO EXEC PGM $=$ TEST, REGION=70OK

176. //STEPLIB DD DSN=CNS376. MI2. NUFS. LOAD, DISP $=$ SHF

177. I/FTO4F001 DD DUMMY

178. I/FTO6FOO1 DD DUMNY

179. //FTO7F001 DD DUMMY

180. //FT08F001 DD DISP=(NEW, PASS), DSN=\&\&TEMP90SR, SPACE $=($ TRK, $(1,1))$,

181. I/ DCB $=$ (RECFM=FB, LRECL=80, ELKSI $E=6160$, DSCFG=PS), UNIT=DASD

182. I/FTO9FOO1 DD *

183. * TITLE OF RUN

184. NUREG METH TEST $6 / 8$

185. * (1) NUMBER OF YRS; (2)NUMBER OF REGIONS; (3)OPT7; (4)OPT8

186. 17251

187. I/FT10FO01 DD ISN=CN6376. MI2. NUREG.PRIVATE(IL), DISP=SHR

186. $/ / \quad$ DD IISN=CNS376.MI2. NLREG. PRIVATE(VA), DISP $=S H R$

189. //FT11F001 DI DSN=CN6376.MI2.NUREG. PUELIC(IL), DISF=SHR

190. $/ / \quad$ DD DSN=CN6376. MI2. NUREG. PUBLIC(VA), DISP $=S H R$

191. I/FT12F001 DO DSN=CN6376.MI2. NUREG.FORM1.PDS78(IL),DISP =SHR

192. $/ / \quad$ DO DSN=CN6376.MI2. NUREG. FDRM1. PDS78(VA), DISP =SHR

193. I/FT13F101 DD DSN=CN6376.MI2. NUREG.FORM1M.PDS78 IIL), DISP=SHR

194. $/ / \quad$ DD DSN=CNE376. MI2. NUREG. FORM1M. PDS78(VA), DISP=SHR

195. . /FT14F001 DD DSN=\&\&TEMP90, DISP=(OLD, DELETE)

196. $1 / *$

197. //STEPRLINA EXEC PGM=TEST, REGION=700K

198. //STEPLIE DD DSN=CNG376. MIZ. NUFS. LOAD, DISP=SHR

199. I/FTO4FOO1 DD DUMAY

200. //FTOGFOO1 DD DUMMY

201. I/FTO7FO01 DD DUMAY

202. I/FTOBFO01 DD DISP $=(N E W, P A S S)$, DSN $=\& \&$ TEMPPSSR, SPACE $=(T R K,(1,1))$,

203. $/ 1 \quad$ ICB $=$ (RECFM=FB, LRECL $=80$, BLKSIZE=6160, DSORG=PS), UNIT=DASD

204. //FTOFFOO1 DD *

205. * TITLE OF RUN

206. NUREG METH TEST $6 / 8$

207. * (1)NUMBER OF YRS: (2)NUMBER OF REGIONS: (3) OPT7; (4)OPT8

208. 17251

209. I/FT10F001 DI DSN=CN6376. MI2. NUREG. PRIVATE(IL), DISP=SHR

210. $/ 1 \quad$ DI DSN=CN6376. MI2. NUREG. PRIVATE (VA), DISP=SHR

211. I/FT11FO01 DU DSH=CNK376. MI2. NUREG. PUBLIC(IL), DISP $=S H R$

212. $/ 1 \quad$ DD DSN=C:NK376. MI2. NUREG, PUELIC(VA), DISP=SHR

213. //FT12F001 DU IGH=CNS376.MI2. NUREG. FORM1.PDS78(IL), DISP=SHR

214. II DD DSN=CN6376. MI2. NUREG. FORM1.PDS78(VA), DISP =SHR

215. I/FTI3F001 DD DSN=CN6376. MI2. NUREG. FORM1M.PDS78(IL), DISP=SHR

216. $/ 1 \quad$ DII DSN=CNG376.MI2. NIREG. FORMIM. PDS78(VA), DISP $=S H R$

217. //FTI4FOO1 DI ISN =\$\&TEMPO5, DISP=(OLD, DELETE)

218. $\quad / 1 \%$

219. I/STEPRINE EXEC PGM=TEST, REGION=70OK

220. //STEPLIE DD LSN=CNE376. MI2, NUFS. LOAD, DISP=SHR

221. I/FTO4FO01 DO DUMMY

222. //FT06F001 DD DUMMY

223. I/FTO7F001 DO DUMAY 
224. //FTOBF001 DU DISP $=($ NEW, PASS), DSN=R\&TEMPALSR, SPACE $=(\operatorname{TRK},(1,1))$,

225. $/ 1 \quad$ ICE $=$ (RECFM=FB, LRECL $=80$, ELKSIZE $=6160$, ISORG $=P S$ ), UNIT $=$ DASII

226. I/FTOFFOOI DO *

227. * TITLE OF RUN

228. NIREG METH TEST \&/S

229. $\quad$ (1)NLMBER OF YRS; (2)NUMEER OF REGIONS; (3)OFT7; (4)OPTO

230. 17251

231. //FT10F001 DII DSN=CNG376.MI2. NUREG.PRIVATE(IL), UISP=SHR

232. $11 \quad$ DI [ISH=CN6376.MI2. NUREG. PRIVATE(VA), DISF $=$ SHF

233. //FT11FOO1 DD DSN=CN6376. ML2, NUREG. PUELIC(IL), DISP $=$ SHF

234. $/ / \quad$ DII ISN=CNS376. MI2.NLREG. PUBLIC(VA), DISP=SHR

235. I/FT12F001 DI DSN=CNG376. MI2. NLREG. FORM1. PDS78(IL), DISP $=S H R$

236. $\quad / 1 \quad$ DD DSN=CNS376. MI2. NUREG. FORM1. PDS78(VA), DISP=SHR

237. //FT13F001 OU DSN=CNG376. MI2. NUREG. FORMIM. PUS78(IL),DISP=SHR

238. $/ / \quad$ DD DSI $=$ CN6376. MI2. NUREG. FORMIM. PDS78(VA), DISP =SHR

239. I/FTI4FOOI DD DSN=\&\&TEMPALL, DISP=(OLD, DELETE)

240. $1 / *$

241. //STEP2 EXEC FORTGCG

242. //FORT. SYSIN DD *

243. C THIS PROGRAM CALCULATES THE SENSITIVITY OF PARAMETERS TO CHANGES IN CAPITAL

244. C. Costs

245. DIMENSION DELCAP(5), BASE $(4,25)$, ALTER $(4,25)$, DEL TA $(4,4,25)$,

246. 1 TARGET (4), ELAS $(4,4,25)$, TOTCAP (5), WGTPEN $(4,25)$

247. CWFIRST READ IN TEMPORARY FILES CREATED FROM FIRST STEP.

248.

249.

250.

251.

252.

253.

254.

255.

256.

257.

258.

259.

260 .

261.

262.

263.

264.

265.

266 .

267.

268.

269.

DOUELE PRECISION NAME1(4)

[LTA NAME1/'INT COV', 'AFUDC \%', 'RT ON EQ', 'EXT FIN\%'/

READ $(8, *)$ NREG

WRITE 20,1$)$ NREG

IO $300 \mathrm{KL}=1$, NREG

READ $(8,50)$ NAME

50 FORMAT (A2)

READ (8,*) RATE

READ(8,*) NYRS1, NYRS2

REAIIS 8 *) TARGET

NYRS=NYRS2-NYRS1+1

DO $230 \quad I=1,4$

230 READ(8, 15) (HGTPEN $(I, \downarrow), J=1$, NYRS)

$\operatorname{READ}(8, *)$ NOSFAN

READ $(8, *)$ (DELCAP(I), I=1, NOSF'AN), (TUTCAP(I), I=1, NOSPAN)

C*NAN PARAMETERS ARE READ IN AND DERIVATIVES ARE CALCULATED. NOSPAN $=$ NOSPAN+1

ITEMP $=$ NOSPAN+1

Do $25 L=1,6$

DO 25 I $=1$, ITEMP

IFILE $=I+9$

270. 26 FORMAT (2A4)

271. REAII $(10, *)$ (IYEAR, (BASE $(J, K), J=1,4), K=1$, NYRS2)

272.

273.

274.

WRITE $(6,120)$ ( $(\operatorname{BASE}(J, K)\rfloor=1,4,), K=1$, NYRSS2)

[10 $10 \quad I=1$, NOSPAN

275.

IFILE $=I+10$

276.

READ(IFILE, *) (IYEAR, (ALTER $(J, K), v=1,4), K=1$, NYRS2)

277.

WFITE $(6,120)$ ((ALTER $(J, K), \downarrow=1,4), K=1, \operatorname{NYRS2})$

278.

Di] $20 \mathrm{~J}=1,4$

279.

$0030 \mathrm{~K}=1$, NYRS2

DELTA $(I, J, K)=(A L T E R(d, K)-\operatorname{BASE}(J, K)) /($ RATE $(-1.0))$ 


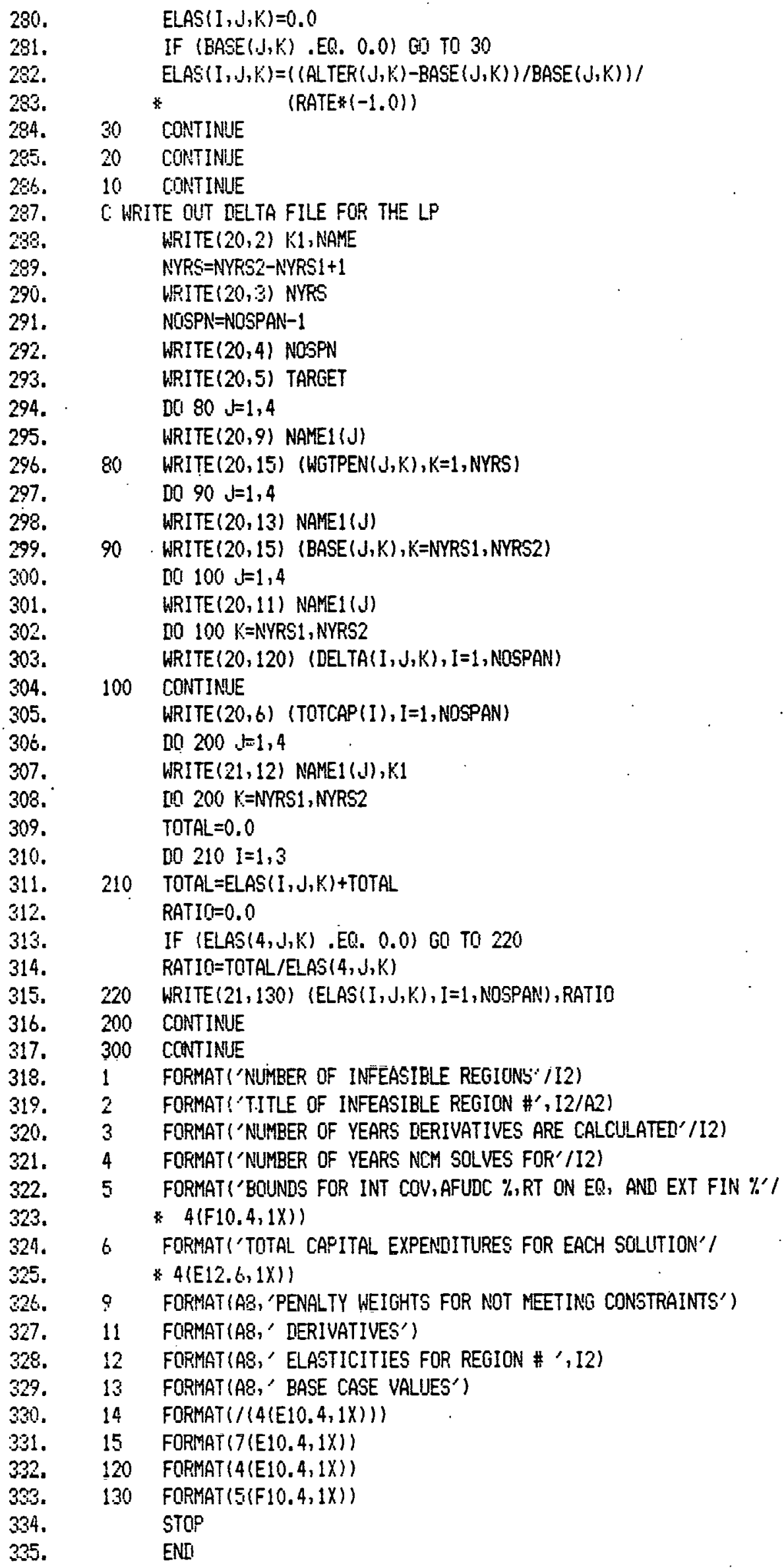


336. //G0.FTO6F001 DU DUMMY

337. //GO.FTOBF001 ID DISP=1OLD, IELETE), DSA=\&\&DELCAP

33.. $\quad / / 60 . F T 10 F 001$ DO DSN=\&\&TEMPBSSR,DISP=(OLD, DELETE)

339. $/ / 60 . F T 11 F 001$ DD DISP=(OLI, DELETE $),$ DSN=\&\&TIYPSSSR

340. $/ /$ GO.FT12F001 DI DISP=(OLD, DELETE), DSN=\&\&TEMPSOSR

341. $/ / 60$. FT13F001 DII IISP = (0LD, IELETE); DSN=\&UTEMPF5SR

342. //GO.FT14F001 DUI IISF $=($ OLDI, DELETE), DSN=\&\&TEMFALSF

343. $\quad / / 60$. FT20FOO1 DD SYSOUT $=A$

344. $\quad / / G 0 . F T 21 F 001$ DI SYSQUT=A 


\subsection{THE CAPACITY ADDITIONS ADJUSTMENT PROGRAM}

This program finds the changes in capital additons that are required to make the NCM solutions financially feasible. It solves region by region and writes files that are read by the NCM revise programs. These files contain upper bounds on the aggregate capital additions for each region under consideration. There are two output files: a file for the 1985 revise, and a file for the $1990 / 1995$ revise. Data from FSP are read from unit 20 . The code links to a load library that contains the linear programming package XMP.

Figure 3 shows the organization of the program.

FIGURE 3

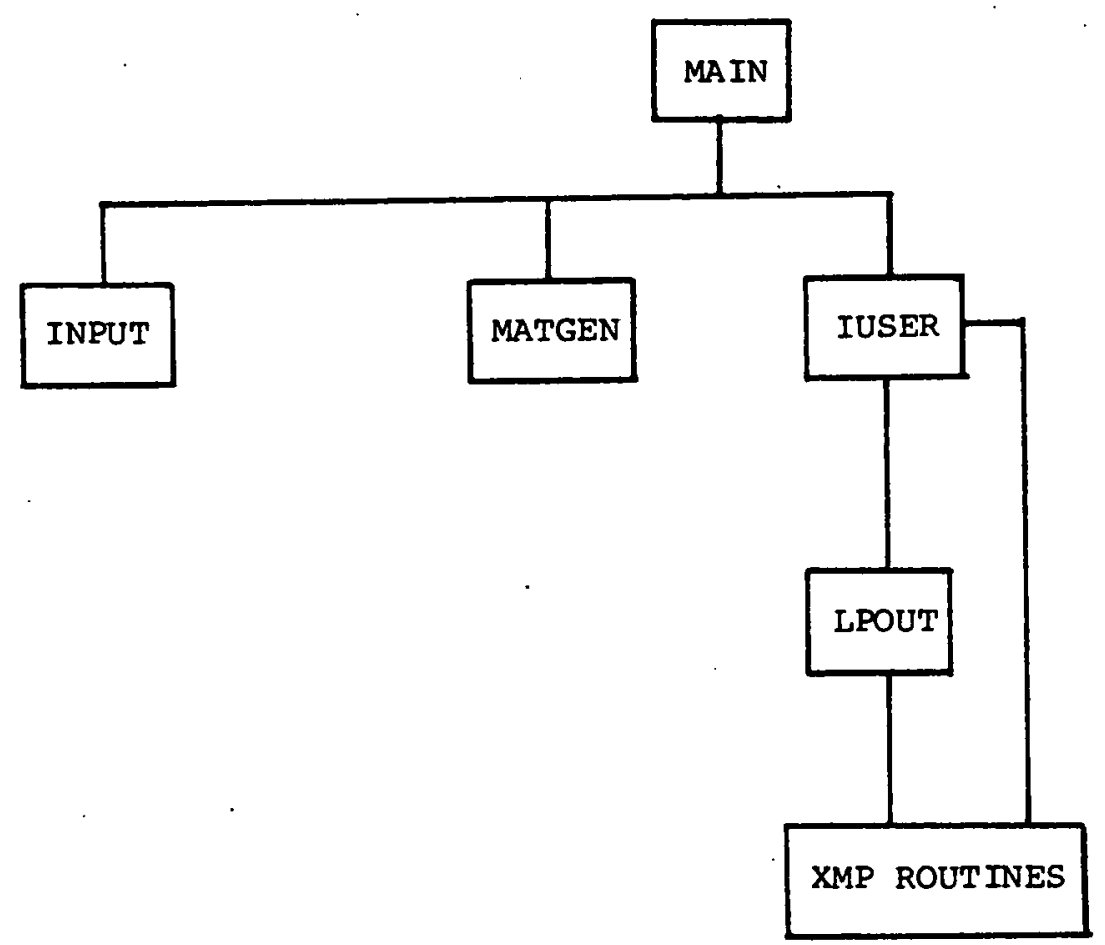


The main program controls the flow of the program and outputs the files to be read by NCM's revise programs. Subroutine INPUT is called to read the data passed by FSP. These data include initial and target values for the financial parameters. The partial derivatives of the financial parameters with respect to changes in capital outlays, and the names of the regions under consideration.

For each region, subroutine MATGEN is called to create a matrix file. Subroutine IUSER is called to solve the linear programing problem LPOUT, which is called by IUSER, extracts the solution data. The main program calculates the new capital bounds and outputs these numbers.

A listing of the program now follows. 
1. CTHIS PROGRAM IS THE MATRIX GENERGTOR FOR THE STEP THAT DETERMINES

2. C A FEASIBLE SET OF CAPITAL GOUNDS. IRA SHAVEL, ICF, INC.

3. $c$

4. REAL $* 4$ REGNAM

5. REAL $\$ 4$ II, OLDPAR, TARGET

6. COMMON /LPDATA/ REGNAM, D $(4,4,21)$, NYR1, NYR2, OLDPAR $(4,21)$, TARGET $(4)$,

7. + BNII (4), ESC(4), HEIGHT(4), PENAL $(4,21), \operatorname{BASE}(4)$, NLMREG

8. REAL $\$ 4$ REGNAM

9. $\quad$ C

10. REAL $\$ 8$ MEMORY $(9000)$

11. REAL $* 8$ TMAX, OBJECT

12. REAL $\$ 4$ VAL $(9000)$, ALAM (100), NEWO5, NEW90, NEW95, DEL 85 , DEL 90, DEL 95

13. DATA OBJECT/'OBJ'I

14. DATA TMAX/-1.DOI

15. IPASS $=0$

16. IF $55=85$

17. IF $90=90$

i8. IF95 $=91$

19. $\quad$ WRITE(IFS5, 1) NUMREG

20. WRITE(IF85,2)

21. WRITE(IF90,3) NUMREG

22. WRITE(IF90, 4)

23. WRITE (IF95,5) NUMREG

24. URITE(IF95,6)

25. KFILE $=99$

26. DO $100 L L=1$, NUAREG

27. REWIND 5

28. CALL INPUT(IPASS)

29. CALL MATGEN

30. REUIND 5

31.

32.

33.

34.

35.

36.

37.

38.

39.

40.

41.

42.

43.

44.

45.

46.

47.

48.

49.

50.

51.

52.

53.

54.

55.

CALL IUSER (VAL, ALAM, OBJECT, TMAX, MEMORY)

DEL $85=-V A L(1)+V A L(4)$

DEL $9(1)=-V A L(2)+V A L(5)$

IELL $95=-V A L(3)+V A L(t)$

NEWBS $=(1 .-D E L 85) \times B A S E(1) / 1000$.

NEW90 $=$ NEWB5 $+(1 .-D E L$.90) $*$ BASE (2) $/ 1000$.

NEW95 $=N E W 90+(1,-D E L 95) * B A S E(3) / 1000$.

WRITE(IF85,7) REGNAM, NEWBS

WRITE (IF90,7) REGNAM, NEW9O

WRITE(IF95, 7) REGNAM, NEW95

100 CONTINUE

1 FORMATI (I2,'OITABLE CAPBD')

2 FORMAT! ${ }^{\prime}$ * CPBDES')

3 FORMAT(I2, '01TABLE CAPBD $\left.\% 0^{\prime}\right)$

4 FORMAT! $\quad$ (PBD90')

5 FORMAT(I2,'O1TABLE CAPBD95')

6 FORMATI' * CPBD95')

7 FORMAT $(9 X, A 4,4 X, F 10.3)$

STOP

END

SUBROUTINE MATGEN

C* THIS ROUTINE WRITES OUT THE MATRIX

c.

REAL *4 D, OLDPAR, TARGET

COMMON /LPDATA/ REGNAM, $D(4,4,21)$, NYR1, NYR2, OLDPAFi $(4,21)$, TARGET(4), 
56. + BND (4), ESC (4), WEIGHT (4), PENAL (4, 21), BASE (4), NUMREG

57. REAL $\$ 4$ REGNAM

58. DIMENSION RTYPE (4), RNAME (5), CNAME (4)

59. DIMENGION CHAR(3)

60. DIMENGION CHAR1 (19)

61. [IATA RTYPE/ ' $N$ ', 'E', 'L', 'G'/

62. DATA RNAME $I^{\prime} I^{\prime},{ }^{\prime} A^{\prime},{ }^{\prime} R^{\prime},{ }^{\prime} E^{\prime},{ }^{\prime} \mathrm{B}^{\prime} /$

63. IATA CNAME/'P', 'M', 'C','S'l

64. DATA CHAR/' $85^{\prime},{ }^{\prime} 90^{\prime}, 195^{\prime} /$

65. DATA CHAR1/'81','82', '83', '84', $85^{\prime},{ }^{\prime} 86^{\prime}, ' 87^{\prime}, ' 88^{\prime}$,

66.

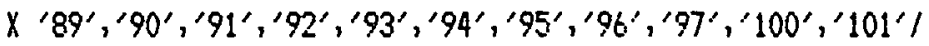

67. C INITIALIE WEIGHT ANI ESC

68. C

69.

70.

71.

72.

73.

74.

75.

76.

77.

78.

79.

80.

81.

82.

83.

84.

85.

86.

87.

C

DO $100 \quad I=1,4$

WEIGHT(I)=.01

$\operatorname{ESC}(I)=1.0$

100 COATINUE

C* $*$ BEGIN WRITING-FIRST THE HEADING ANI ROW SECTION

WRITE $(5,11)$ REGNAM

WRITE $(5,12)$

RT=RTYPE(1)

WRITE $(5,13) R T$

RT=RTYPE(2)

RN=RNAME (5)

DO $201 \mathrm{I}=1$, NYR2

WRITE $(5,14)$ RT, RN,CHAR(I)

201 CONTINUE

RT=RTYPE (3)

RN=RNAME (2)

DO 202 I=1, NYRI

WRITE $(5,14)$ RT, RN,CHARI(I)

202 CONTINUE

RNE=RNAME (4)

89. DO 203 I $=1$, NYRI

90. WRITE $(5,14)$ RT, RN, CHARIII)

91. 203 CONTINUE

92. RT=RTYPE (4)

93. $\quad$ RN=RNAME (1)

94. $\quad$ IO 204 I=1, NYRI

95. WRITE $(5,14)$ RT, RN, CHARI (I)

96: 204 CONTINUE

97.

98.

99.

100.

101.

102.

103.

104.

105.

106.

107.

108.

109.

110.

111.

RN=RNAME (3)

DO 205 I=1, NYRI

WRITE $(5,14)$ RT, RN, CHARI (I)

205 CONTINUE

C.KKKKOLUMASS NEXT

WRITE $(5,15)$

RN=RNAHE (5)

$C N=$ CNAME (1)

VALUE $1=-1$.

DO $301 \mathrm{I}=1$, NYR2

VALUE $=+E S C(I)$

WRITE $(5,16)$ CN, CHAR(I), VALLE

HRITE(5, 28) CN, CHAR(I), FN, CHAR(I), VALLIEI

301 CONTI INUE

RN $=$ RNAME (5) 


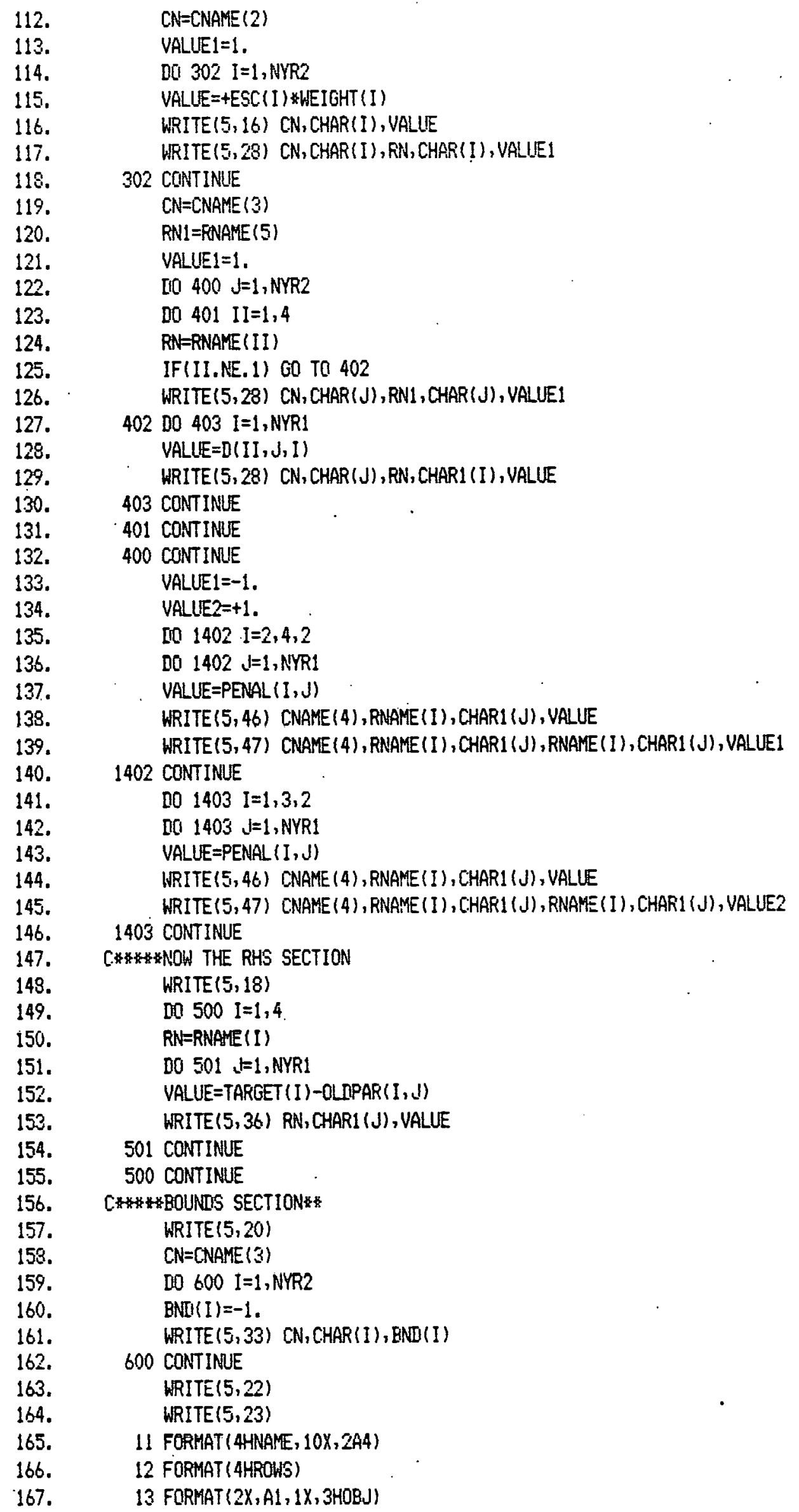


168. 14 FORMAT $(2 X, A 1,1 X, A 1, A 2)$

169. 15 FOFMAT (THCOLUNNS)

170. 16. FORMAT (4X, Al, A2, 7X, 3HOBJ, 7X,F12.5)

171. 18 FORMAT (3HRHS)

172. 20 FORMAT (6HBOUNDS)

173. 22 FORMAT (GHENDATA)

174. 23 FORMAT $(2 H / k)$

175. 28 FORMAT $(4 X, A 1, A 2,7 X, A 1, A 2,7 X, F 12.5)$

176. 33 FORMAT (1X, 2HLO, IX, 3HBD1, 7X,A1, A2, 7X,F12.2)

177. 36 FORMAT ( $4 X, 4$ AHRHS1, $6 X, A 1, A 2,7 X, F 12.5)$

178. 46 FORMAT ( $4 X, A 1, A 1, A 2,6 X, 3 H O B J, 7 X, F 12.5)$

179. 47 FORMAT $(4 X, A 1, A 1, A 2,6 X, A 1, A 2,7 X, F 12.5)$

190. RETURN

181. END

182. SUBROUTINE INPUT(IPASS)

183. C THIS ROUTINE REAIS DATA FROM UNIT 20.

184. REAL $\$ 4$ D, OLDPAR, TARGET

185. COMMON /LPDATA/ REGNAM, D $(4,4,21)$, NYRI, NYR2, OLDPAR $(4,21)$, TARGET $(4)$,

186. + BND(4), ESC (4), WEIGHT (4), PENAL $(4,21)$, BASE(4), NUMREG

187. REAL $\$ 4$ REGNAM

188. IF(IPASS.GT.0) GO TO 25

189. READ $(20,2)$ NUMREG

190. 25 CONTIAUE

191. READ $(20,1)$ REGNAM

192. READ $(20,2)$ NYRI

193. READ $(20,2)$ NYR2

194. $\operatorname{READ}(20,3)(\operatorname{TARGET}(1), I=1,4)$

195. DO $50 I=1,4$

196. READ $(20,5)$

197. $\operatorname{READ}(20,13)$ (PENAL $(1, J),\rfloor=1, N Y R 1)$

198. 50 CONTINUE

199. DO $100 \quad I=1,4$

200. READ $(20,5)$

201. $\operatorname{REAU}(20,13)$ (OLDPAR( $1, J), J=1, \operatorname{NYR1)}$

202. 100 CONTINIE

203. $\quad$ DO $150 \quad I I=1,4$

204. . $\operatorname{READ}(20,5)$

205. $10200 I=1$, NYRI

206. $\quad \operatorname{READ}(20,4)(D(I I, J, I), J=1, N Y R 2)$

207. 200 CONTINUE

208. 150 CONTINUE

209. $\operatorname{READ}(20,6) \cdot(\operatorname{BASE}(1), I=1$, NYR2)

210. 1 FORMAT(/A4)

211. 2 FORMAT (/12)

212. 3 FORMAT $(/ 7($ E $10.4,1 \times))$

213. 13 FORMAT( $7(E)(0,4,1 \times))$

214. 4 FORMAT $(4(E) 10.4,1 \times))$

215. 5 FORMAT(AI)

216. 6 FORMAT $(/ 4(E) 2.6,1 \times))$

217. IPASS=IFASS+1

218. RETURN

219. END

220. SUBROUTINE ILSER(VAL, ALAM, OB.ECT, TMAX, MEMORY)

221. C* ROTHIS ROUTINE SOLVES THE LP. IT CALLS THE XMP KUUUTINES

222. C AND LPOUT.

223. C 
224.

225.

226.

227.

228.

229.

230.

231.

232.

233.

234.

235.

236.

237.

238.

239.

240.

241.

242.

243.

244.

245.

246.

247.

248.

249.

250 .

251.

252.

253.

254.

255.

256.

257.

258.

259.

260.

261.

262 .

263.

264.

265.

266.

267.

268.

269.

270.

271.

272.

273.

274.

275.

276.

277.

278.

279 .
DOURLE PRECISION B(100), BASCB(100), BASLB(100), BASUB(100),

$X$ BETAR(100), BOUND, CANDIA $(90,6)$, CANDCU(6), CU, COLA(90),

$X$ LU, MEMORY (1), UL, UZERO(100), XBZERO(100), YQ(100), Z

INTEGER BNDTYP, COLLEN, COLMAX, DFEASQ, DTERM, DUNBR,

$X$ FACTOR, IOERR, IULOS, ITER,

$X$ ITER1, ITER2, LENHA, LENMI, LENMY, LOOK, M, MAPA $(90)$,

$X$ MAPI (90), MAXA, MAXM, MAXN, N, NTYPE2, P, PRINT, TERMIN, UNBDDG

INTEGER $\because 2$ CAND $(6)$, CANDI $(90,6)$, CANDL $(6)$,

$X$ COLI $(90)$, ROWTYP $(100)$

COMMON/XMPCOM/BIG, SMALL, ZL, ZLC, EPS1, EPS2, EPS3, EPS4, EPS5, EPS6

COMMON /XINTG2/ STATUS(2800), BASIS (100), ZS(2800), ZB(100)

INTEGER $* 2$ STATUS, BASIS, ZB, ZS

REAL $* 8$ BIG, SMALL, ZL, ZLC, EPS1, EPS2, EPS3, EPS4, EPS5, EPS6

REAL $* 8$ ROLNAM(100), COLNAM (2800), ORUECT, TMAX

COMMON/XINTG4/SLACKH,SLACKL, N,M

INTEGER SLACKL, SLACKH

REAL $\$ 4$ VAL (1), ALAM(1)

IOIN $=5$

IOERR $=6$

$10 L 06=6$

MAXA $=5000$

MAXY $=100$

MAXN $=1000$

COLMAX $=90$

$P=6$

LOOK $=200$

FACTOR $=50$

LENAY $=9000$

PRINT $=1$

BNDTYP $=4$

WRITE $(6,9001)$

9001 FORMATI' IN IUSER')

C. IF(NPASE.GT.1) GO TO 6.91

CALL XMAPS (BNDTYP, IOEFR, LENMA, LENMI, LENMY,

$X$ MAPA, MAPI, MAXA, MAXM, MAXN, MEMORY)

691 CALL MPSXINIM, N, B, ROKTYP, COLMAX, LENMA, LENMY, MAPA, MEMORY

$X$, IOERR, BNDTYP, COLLEN, COLA, COLI, CJ, LJ, LJ

$X$, IOIN, OBJECT, ROLNAM, COLNAM, TMAX)

SLACKL $=N+1$

C. IF(NPASS.GT.1) 60 TO 692

DO $170 \quad \mathrm{~N}=1, \mathrm{~N}$

$170 \operatorname{STATUS}(\mathrm{J})=0$

CALL XSLACK IB, BASCB, BASIS, BASLB, BASUB, BNDTYP, BIUND,

$X$ COLA, COLI, COLMAX, IOERR,

$X$ LENMA, LENMI, LENAY, M, MAPA, MAPI, MAXM, MAXN, MEMORY,

$X N$, ROWTYP, STATUS,

$X$ UZERO, XBZER $(1, Z)$

SLACKH=N

1000 CALL XPRIML (B, BASCB, BASIS, BASLB, BASUE, BNDTYP, BOLND,

$X$ CANI, CANDA, CANDCJ, CANDI, CANDL,

$X$ COLA, COLI, COLMAX,

$X$ FACTOR, IOERR, IOLOG, ITER1, ITER2,

$X$ LENMA, LENMI, LENAY, LOOK,

$X$ M, MAPA, MAPI, MAXM, MAXN, MEMORY,

$X$ N, NTYPE2, P, PRINT, STATUS, TERMIN, UNBDLC,

$X$ UZERO, XBZERQ $Y Y(, Z Z)$ 
280.

281.

282.

283.

284.

265.

286.

287.

288.

289.

290.

291.

292.

293.

294.

295.

296.

297.

298.

299.

300.

301.

302.

303.

304.

305.

308.

307.

308.

$30 \%$.

310.

311.

312.

313.

314.

315.

316.

317.

318.

$31 \%$.

320.

321.

322.

323.

324.

325.

326.

327.

328.

329.

330.

331.

332.

333.

334.

335.
IOLOS $=10$

WRITE(IOLOG,908) TERMIN , $M, N$

908 FORMAT (18H TERMINATION CODE $=, 14,6 \mathrm{H}$ ROHS, $14,6 \mathrm{H}$ COLS, I4)

CALL LPOUTIVAL, ALAM, BASIS, ENUTYF, BOIND, B,

$X$ IOERR, IOLOG,

$X$ LENMA, LENMY, M, MAPA, MAXM, MAXN, MEMORY, N, NTYPE2,

$X$ STATUS, XEZERO, $Z$, UZERO, ROWNAM, COLNAM, SLACKL, SLACKH, TMAX)

CALL MPSXOTIBASIS, BNDTYF, BOUND, B,

$X$ IOERR, IOLOS,

$X$ LENMA, LENMY, M, MAPA, MAXM, MAXN, MEMORY, N, NTYYEZ,

$X$ STATUS, XBZERO, $Z$, UZER0, ROHNAM, COLNAM, SLACKL, SLACKH, TMAX)

IF (TERMIN. EQ.3) STOP

RETURN

ENII

SJIBROUT INE LPOUT IVAL, ALAM, BASIS, BNDTYP, BOUNI, B,

C* THIS ROUTINE READS THE LP SOLUTION.

C

$X$ IOERR, IOLOG,

$X$ LENMA, LENMY, M, MAPA, MAXM, MAXN, MEMORY, N, NTYPE2,

$X$ STATUS, XBZERO, Z, UZERO, ROLNAM, COLNAM, SLACKL, SLACKH, TMAX)

INTEGER LENMA, LENMY, MAXM, MAXN

DOUBLE PRECISION BOUND, MEMORY (1), XBZERO(1),2,21

REAL 8 UZERO(1), ROWNAM(1), COLNAM(1), B(1)

REAL $\div 8$ TMAX, TZ

INTEGER BNDTYP, IOERR, IOLOG, M, MAPA(1), N, NTYPE2

INTEGER SLACKL, SLACKH

INTEGER $\approx 2$ BASIS(1), STATUS(1)

DOUBLE PRECISION LU,UJ, VALUE

INTEGER IX,d

REAL $\$ 4$ VAL (1), ALAM(1)

NUP $=$ SLACKL -1

Z1=Z/TMAX

C. WRITE $(50,776)$ II

776 FORMAT(' OPTIMAL OBJECTIVE VALUE $=$ ', E14.6)

DO $10 \quad I=1, M$

10 ALAMI I $)=U$ UZERO(I) /TMAX

C WRITE $(5,777)$ (I, ALAM(I), I I I,M)

777 FORMAT' DUALS'/(5)(15,E14.6)))

DO $280 \mathrm{~J}=1$, NUP

IF (STATUS(J)) $200,250,260$

200 IF (STATUS(J).EQ. -2 .OR. STATUS(J).EQ. -3)GO TO 230

IF(STATUS(J).EQ. -4$) 60$ TO 250

IF (BNDTYP.LT.3)G0 TO 210

CALL XGETUB (BNDTYP, 10ERR, J, LENMA, LENMY, LJ, MAPA, MEMORY, LU)

VAL $(J)=U J$

GO TO 230

210 IF (BNDTYP.LT.2) 60 TO 220

IF(J.GT.NTYPE2) 200 TO 220

VAL $(J)=$ BOUND

GO TO 280

220 URITE(IOLOG,905) I

905 FORMAT (16H ERROR: VARIABLE, I6, 16H IS AT ITS UPPER,

$X 20 \mathrm{H}$ BOUND OF +INFINITY.)

60 TO 280

$23000240 \quad I=1, M$

IF(BASIS(I) .NE. J) 60 TO 240 


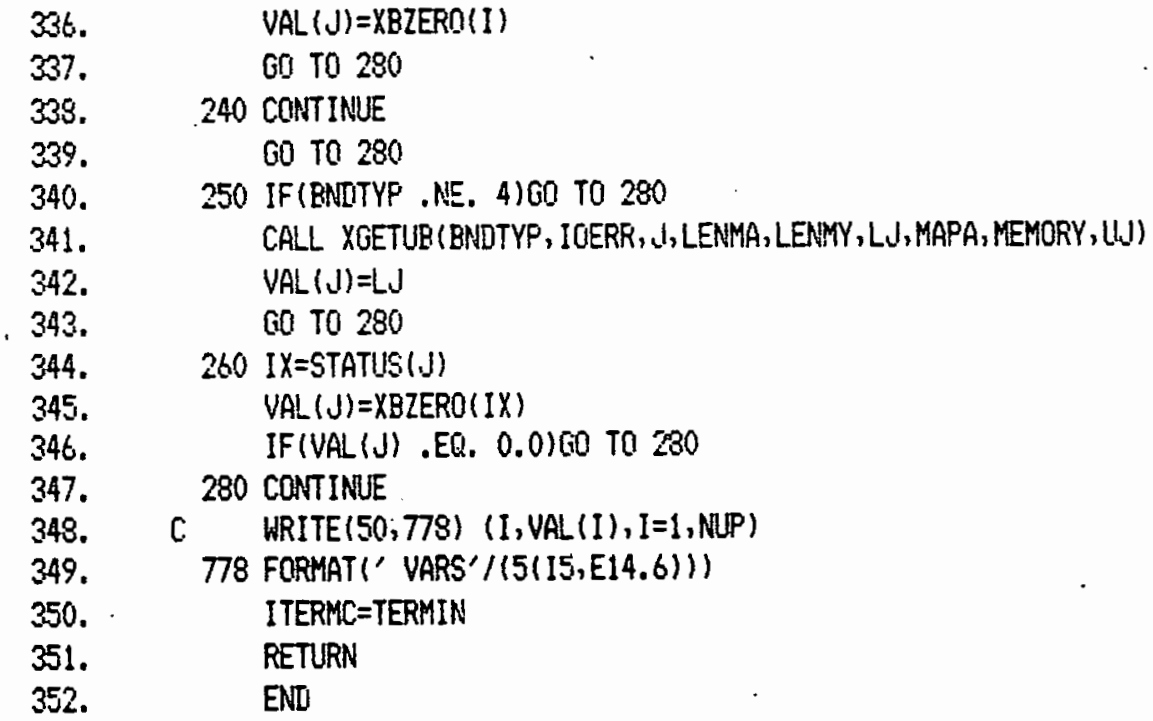




\subsection{NUREG:FORMI.FORT}

NUFS requires historical base year financial statement information for each NCM region. To provide this input for private utilities, NUREG. FORMI. FORT has been created. The primary input is a data tape which contains the base year financial statements for every private utility. This tape is produced annually by the Energy Information Administration. The program aggregates the data for all utilities in a given region and creates a file for each NCM region containing the results. It is very similar in structure to programs created to aggregate data to the MEFS/DOE regions.* The aggregation technique is discussed in Appendix $A$ of Volume 1.

The program consists of a main routine and one subroutine entitled FORMl. The main subroutine reads the tape and aggregates the financial accounts to NCM regions. Subroutine FORMI then calculates ratios and creates files containing the results for NUFS. The remainder of the program is a series of job steps which create a partitioned data set containing the data sets for NUFS. A listing now follows.

* The user is referred to the user's guide for NUFS for additional information on running this program. 
1. I/MI2UFORM JAES $(6376, D 02,999),{ }^{\prime}$ FORM1', TIME $=(1,30)$, MSGCLASS $=A$

2. I/FPC EXEC FORTGCO; REGION=900K

3. I/FORT.SYSIN DQ *

4. DIMENSIDN ISTATE (60), IAFO(300), IEXCP (110), IREGS $(110,4)$,

5. * PERCEN $(110,4)$, NOREG $(110)$

6. IOUELE PRECISION AMNT, AMNT1, AMOUNT (45, 300), AMNT2(300)

7. RERL 44 ACODE, ITYP, KTYP, LTYF, MTYP, NTYF, OTYP, PTYF, OTYP,

8. $\quad$ RTYF, RBKTYP, NOTYF, NITYF, N2TYF, N3TYP, NATYP, NSTYP, NETYP,

9.

10.

* NTTYP, NBTYP, NOTYF

DATA JTYP $/ J \%$

DATA KTYP/'K'I

IIATA LTYP/'L'I

DATA MTYP/'M'I

DATA NTYP/'N'I

DATA OTYP $/$ 'O' $/$

DATA PTYP/'P'I

DATA QTYP/'Q'I

DATA RTYP/'R'I

19. C DATA RBKTYPP $/ \circ \% 1$

20. DATA NOTYP $/ 10^{\prime} /$

21. DATA NITYP $/ 1 \%$

22. IATA N2TYP $/ 22^{\prime} /$

23. DATA N3TYPI' 31

24. DATA N4TYP $/{ }^{\prime} 4 /$

25. DATA NSTYP/'5'/

26. IIATA NGTYP/'G'I

27. DATA N7TYP/'7'I

28. DATA NETYP/'8\%

29. DATA NOTYP/' $9 \% 1$

30. C* *NUREG. FOFMI. FORT. THIS PROGRAM READS DATA FROM THE FORM I TAPE AND

31. C. WURITES OUT DATA NEEDED FOR THE NATIONAL UTILITY REGULATORY

32. C**MODEL (NIREG).

33. C* C**

34. C**WHITTEN BY MARK R. INGLIS, ICF INC. DEC 31,1980

35. C\&*

36. CKHFIRST, A USER SIPPLIED INPUT FILE IS READ. THIS FILE CONTAINS STATE

37. C $\because A N D$ ACCOUNT CODES.

38. C NSTATE=\# OF STATES

3. C NINFO= \# OF ACCOUNTS TO BE ADDDED OVER REGIONS

40. ISTATE(I)=DOE REGION THAT STATE I IS IN

41. C INFO(I) $=6$ DIGIT ACCOUNT CODE

42. C NEXCP=\# OF UTILITIES WHICH ARE SPLIT ACROSS REGIONS

43. C IEXCP= III \# OF SPLIT UTILITY

44. C $\quad$ NORES = \# OF NCM REGIONS WHICH UTILITY SPLITS ACROSS

45. C (IREGS(I, J), Jel, NOREG(I)) = THE COIES OF THE REGIONS WHICH

46. CW SPLIT THE UTILITY.

47. CW (PERCEN $(I, J), J=1$, NORECI $(I)=$ THE \% SPLITS CURRESPONDING TO

48. CW IREGS REGICNS.

4\%. READ(9; * NSTATE

50. DO 77, IN N,NTATE

51. 77 REA[(9,*) I,ISTATE(I)

5\%. READ (9,*) NINFO

53. IIO 88 I=1, NINFO

54. 88 READ $(9,802)$ INFO(I)

55. 802 FOFMAT(I6) 


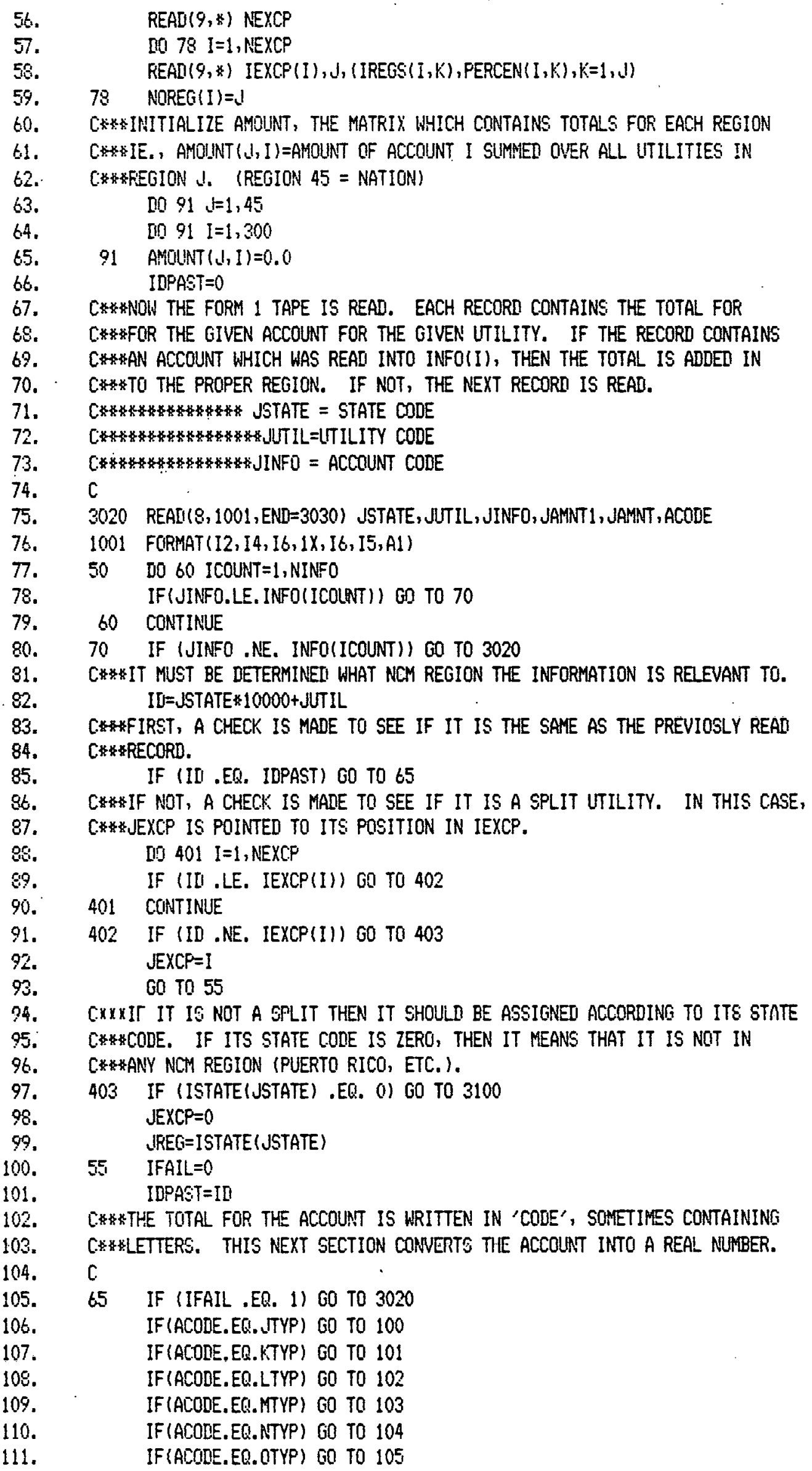


112. IF (ACODE.EQ.PTYF) GO TO 106

113. IF (ACODE. EQ. OTYP) 60 TO 107

114. IF (ACOUE. EQ. RTYP) GO TO 108

115. C: IF (ACCOEE. EQ.RBKTYP) COO TO 109

116. IF (ACOIE. EQ. NOTYP) GO TO 110

117. IF(ACODE.EQ.NITYP) GO TO 111

118. - IF (ACODE. EQ.N2TYP) GO TO 112

11\%. IF (ACODE. EQ.NITYP) GO TO 113

120. IF (ACOIE. EQ.N4TYP) GO TO 114

121. IF (ACOUE. EQ.NSTYP) GO TO 115

122. IF (ACODE. EQ.NETYP) GO TO 116

123. IF (ACODE. EQ.NTTYP) GO TO 117

124. IF (ACODE. EQ. NBTYP) GO TO 118

125. IF (ACODE. EQ.NOTYP) GO TO 119

126. . COTO 109

127. C

128. 100 JAMNT $=-10 *$ JAMNT -1

129. $\quad$ GO TO 900

130. 101 JAMNT $=-10 *$ JAMNT -2

131. 60 TO 900

132. 102 JAFNT $=-10$ * JAMNT -3

133. $\quad$ GO TO 900

134. 103 JAMNT $=-10 *$ JAMNT -4

135. $\quad G 0$ TO 900

136. 104 JAFNT $=-10 *$ JAMNT -5

137. $\quad 60$ TO 900

138. 105 JAMNT $=-10 *$ JAMNT-6

139. GO TO 900

140. 106 JAMNT $=-10 *$ JAMNT -7

141. GO TO 900

142. 107 JAMNT $=-10 *$ JAMNT -8

143. $\quad 60$ TO 900

144. 108 JAMNT $=-10 *$ JAMNT -9

145. GO TO 9.00

146. 109 JAMNT $=-10 *$ JAMNT

147. $\quad$ GO TO 900

148. 110 JAMNT=10*JAMNT

149. GO TO 900

150. 111 JAMNT $=10 * \Delta A M N T+1$

151. G0 TU $\% 0$

152. 112 JAMNT $=10 *$ JAMNT +2

153. 60 TO 900

154. 113 JAMNT $=10 *$ JAMNT +3

155. GO TO 900

156. $114 \quad$ JAMNT $=10 *$ JAMNT +4

157. $\quad 60$ TO 900

15. $115 \quad$ JAMNT $=10 *$ JAMNT +5

159. 60 TO 900

160. $116 \quad$ JAMNT $=10 *$ LAMNT +6

161. $\quad 60$ TO 900

162. 117 JAMNT $=10 *$ JAMNT +7

163. $\quad 60$ TO 900

164. 118 JAMNT $=10 *$ JAMNT +8

165. GO TO 900

166. 119 JAMNT =jo*JAMNT +9

167. $\quad 6050900$ 


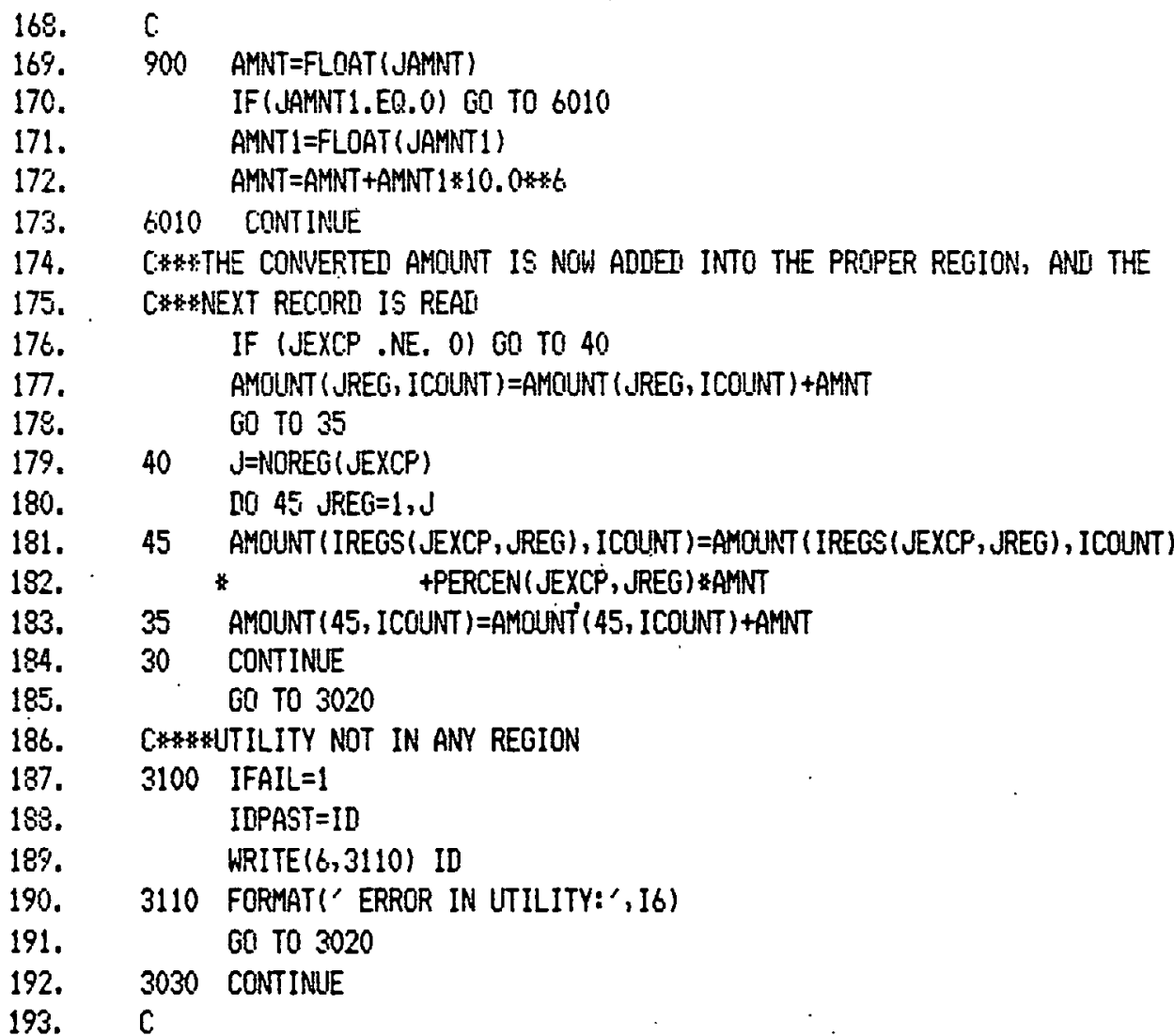

194. C**AFTER THE WHOLE TAPE HAS BEEN READ, SLIBROUTINE FORMI IS CALLED FOR 195. C* $*$ EEACH REGION. THIS SUBROUTINE WRITES OUT THE DATA NECESSARY FOR THE 196. C* *NUFS MODEL.

197. nO 950 IREG $=1,45$

19.. DO $940 \mathrm{I}=1, \mathrm{NINFO}$

199. 940 AMNT2(I)=AMOUNT (IREG, I)

200. CALL FOFM1 (IREG, AMNT2)

201. 950 CONTINUE

202. $5000 \quad 5 T O P$

203. END

204.

205 .

206.

207.

208.

209.

210.

211

212

213.

214.

215.

216.

217.

SUBROUTINE FORMI(IREG, AMOUNT)

$$
\text { C }
$$

C*

$c *$

SUBROUTINE FORMI

THIS SUBROUTINE TAKES THE TOTALS FROM THE MAIN PROGRAM FOR EACH REGION AND WRITES OUT FILES CON-* TAINING THE DATA NEEDED BY NIFS. IT ALSO PRINTS * OUT VARIOUS QUANTITIES OF INTEREST TO UNIT 6 . * (UNIT GELINE PRINTER) 


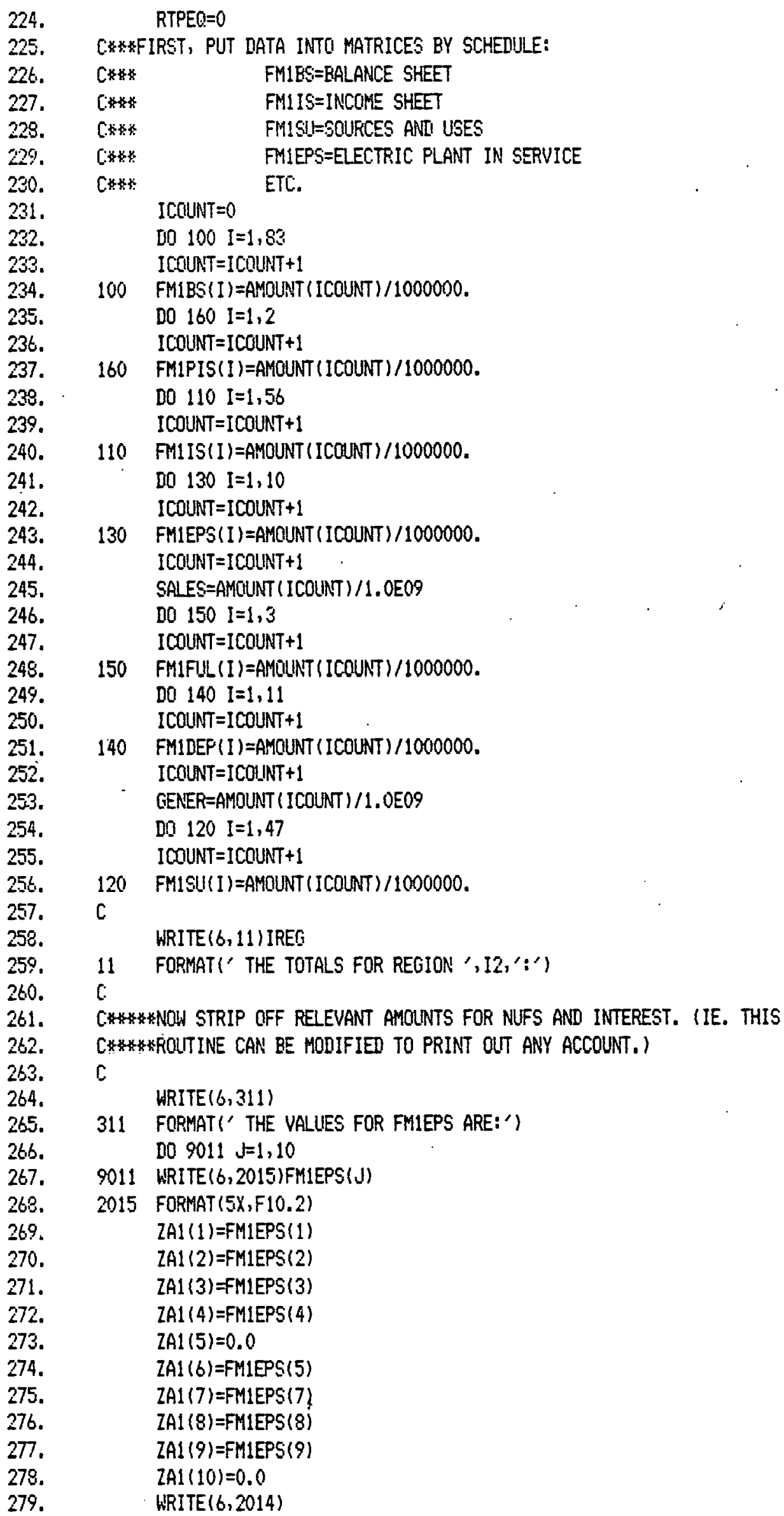




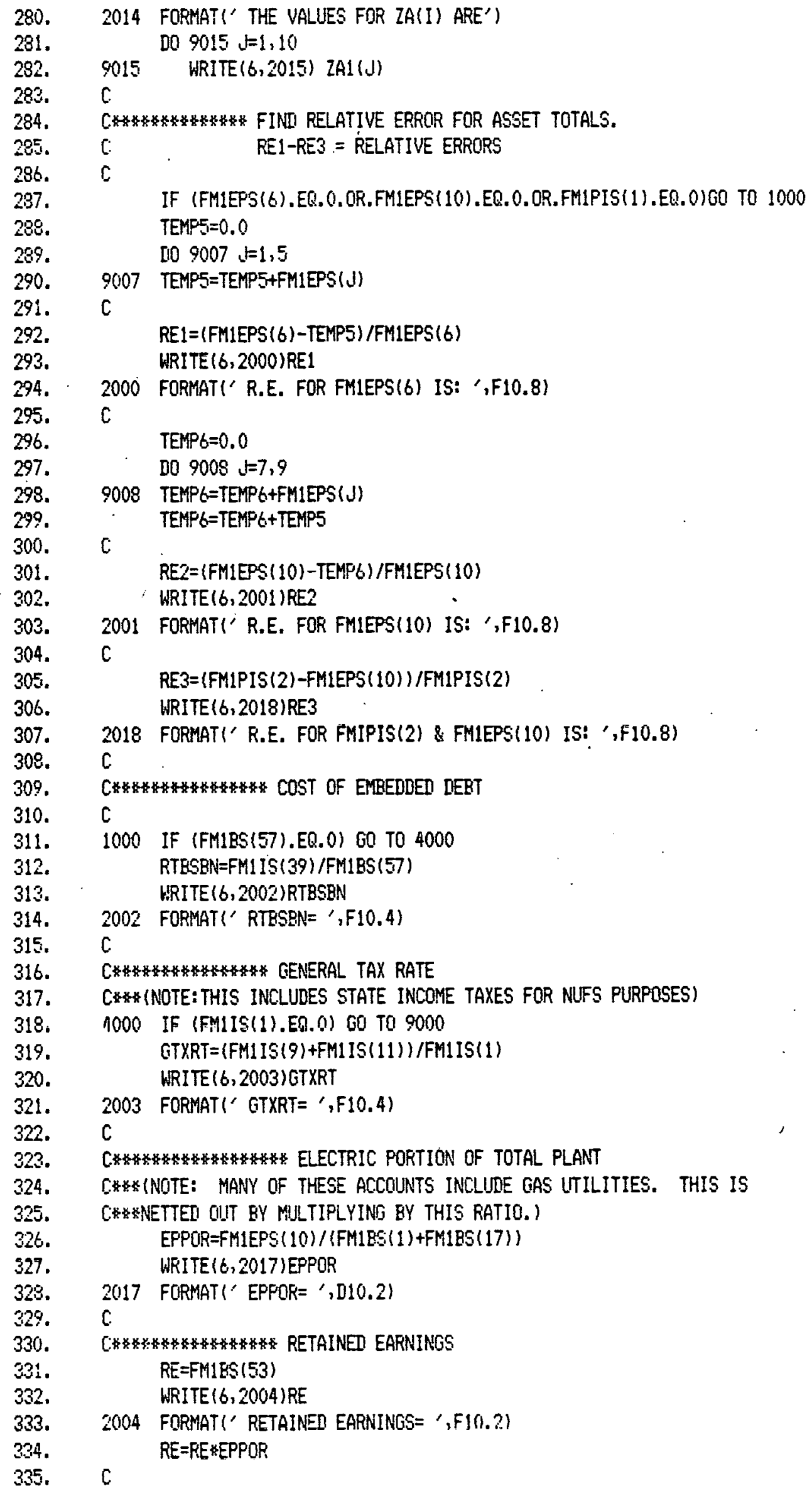


336. C. STOCK OF OUTSTANDING BONDS PRE-BASE YEAR

337.

338.

C

(3). QREFMIBS(62)

339. WRITE $(6,2005) 0 \mathrm{E}$

340. 2005 FORMATI" OUTSTANDING BONIS = ',F10.2)

$341 . \quad O E=0 \mathrm{~B} * \mathrm{EPPOR}$

342. C*

343.

344. STDERT $=$ FM1BS $(6.3) *$ EPPOR

345. 2050 FORMATI' SHORT TERM IEBT $=', F 10.21$

346. $C$

347. C

348. C

349. REVS=FMIIS(1)

350. WRITE(6, 2006) REVS

351. 2006 FORMATI' ELECTRIC REVENUES = ',F10.21

352. C

353. C CAPITAL STRUCTURE

354. C

355. $\therefore \quad$ TEMP7=FM1BS (56)+FM1BS(62)+FM1BS(63)

356. $W 1=F M 1 B S(62) / T E M P 7$

357. WRITE(6,2007) W1

358. 2007 FORMATI' WI $=$ ',F10.4)

359. W2=FM1BS(63)/TEMP7

360. WRITE $(6,2003)$ W2

361. 2008 FORMATl' W2 $=$ ',F10.41

362.

363.

364.

365.

366 .

367.

368.

369.

370.

371.

W3 $=$ (FM1BS(56)-FMIBS(45))/TEMP7

WRITE $(6,2009)$ W3

2009 FORMAT!' W3 = ',F10.4)

W4=FMIBS(45)/TEMP7

WRITE $(6,2010)$ W4

2010 FORMATI' $W 4=', F 10.4)$

c

C* TDTAL ACCUMULATED DEFFERED ITC

C

372. WRITE(t, 2011)TDITC

373. 2011 FORMATl' TOTAL DEFERED ITC $=$ ',F10.2)

374. IUIT:=TUITCEEPPOR

375. $\quad$ C

376. C ACCUMULATED DEFFERD I/T

377.

378.

379 .

380.

331.

392.

383.

334.

35.

386.

387.

338. C DIVIDEND PAYOUT RATIO

33\%. CH (NOTE: THIS IS \% OF EARNINGS AVAILABLE TO COMMON, NOT NET INCOME)

390. $\quad$ DIVRAT $=F M 1 I S(56) /(F M 1 I S(54)-F M 11 S(55))$

391. WRITE $(6,2023)$ IIVRAT 
392. 2023 FORMATl' DIVIDEND FAYOUT RATIO= ',F10.4)

393. $\quad \mathrm{C}$

394. CW WIRKING CAPITAL

395. C

396. $W(=\{F M 1 B S(31)-F M 1 B S(74))$

397. WRITE $(4,2021)$ WC

398. 2021 FIGMATI' WIRKING CAOITAL = ',F10.41

39. WE WCKEPFOR

400. C

401. C. CW COMON STOCK PRE-EASE YEAR

402. C

403. $\quad$ COMSTK $=($ FMIBS(56) $-F M I B S(53)-F M 1 B S(45))$

404. WRITE $(6,2022)$ COMSTK

405. 2022 FORMAT(' COMMON STOCK $=', F 10.4$ )

406. COMSTK=COMSTK $*$ EPPOR

407. C

408. C PREFERRED STOCK PRE-BASE YEAR

409. $\quad C$

410. PRESTK=FM1BS(45)

411. WRITE $(6,2024)$ PRESTK

412. 2024 FORMATl' PREFERREI STOCK = ',F10.4)

413. FRESTK=PRESTK. EPPOR

414. $\quad C$

415. C RETURN ON PREFERRED EQUITY

416. $\quad 6$

417. IF (FMIBS(45).EQ.0) GO TO 3000

418. RTPER =FM1IS(55)/FM1BS(45)

419. WRITE $(6,2020)$ RTPEQ

420. 2020 FORMATI' RETURN ON PREFERRED EQUITY= ',F10.4)

421. C

422. C CONSTRUCTION WORK IN PROGRESS ACCOINT

423. C

424. 3000 WRITE(6, 2025) FM1ES(2)

425. 2025 FORMAT(' CWIP ACCOUNT:',F10.2,'1')

42b. C

427. C NET INCOME

428. C

42\%. ESHRI $=$ FM1IS(18)/FM1IS(19)

430. WRITE $(6,2026)$ ESHRI

431. 2026 FORMAT(' ESHRI $=\prime, 1 \times, F 10,4)$

432. ENETIN=ESHRI KFM1IS(54)

433. WRITE $(6,2028)$ ENETIN

434. 2028 FORMAT(' ELECTRIC NET INCOME (NET OF GAS) $=$ ',F10.4)

435. C C

436. C*NNOW WRITE RESULTS TO FILES FOR NUFS.

437. $\quad$ C****:

438. 9000 NNN=IREC +19

439. CW WRITE OIIT BASE VEAR GENERATION

440. WRITE(NNN, 200) GIENER

441. 200 FORMAT(E15.9, 5X, 'GENERATION')

442. WRITE $(6,200)$ GENER

443. C* WHITE OIT BASE YEAI SALES

444. WRITEINN, 210) SALES

445. 210 FORMATIEI5.9,5X, 'SALES TO LLTIMATE')

446. WRITE $(6,210)$ SALES

447. C*ANITIE OUT EXISTING ASSET BASE 
448.

449.

220 FORMAT(E15.9, 5X, 'INTANGIBLE (EXISTING)'/E15.9,5X,'STEAM PROOU',

450. 1 'CTION'/E15.9, 5X, NILLEAR PRODUCTION'/EI5.9,5X, 'HYRO CONV',

451. 2 E15.9,5X, 'PUMPEU STORAGE'/E15.9, 5X,'OTHER PRODICTION'/E15.9,

452.

45. 3 5X,'TRA:IGMISIION'/EI5.9, 5X,'DISTRIEUTION'/E15.9,5X,'GENERAL'/

454.

4 E15. $8,5 X_{2}$ 'COMMON-ELE!')

455.

CKR:WRITE QUT EASE YEAR STOCK OF BONIS

WRITE (WNWN, 230) OB

230 FORMAT (E15. $\%, 5 \%$, OUTSTANDING BONIS')

457. CK\$LITE OUT BASE YEAR SHORT TERM IEET

458. WRITE(NAN, 240) STUEBT

459. 240 FORMAT(E15.9,5X,'SHORT TERM DERT')

460. C* *WRITE OUT EASE YEAR COMMON STOCK

461. WRITE(NNN, 250) COMSTK

462.

463.

464.

465.

466.

467. WRITE(NNN, 270) RE

FORMAT (E15.9,5X,'COMMON STOCK')

C**WRITE OUT BASE YEAR PREFEREI STOCK

WRITE (WNN, 260) PRESTK

260 FORMAT (E15.9, 5X, 'PREFERRED STOCK')

468. 270 FORMAT (E15.9,5X, 'RETAINED EARNINGS')

46\%. C**\#URITE OUT BASE YEAR EMBEDDED COST OF LONG TERM DEBT

470. WRITE(NNA, 280) RTBSEN

471. 280 FORMAT(E15.9,5X,'COST OF EMBEDDED DEBT')

472. C**WRITE OUT BASE YEAR EMBEDDED COST OF PREFERRED STOCK

473. WRITE (NNN, 285) RTPEU

474. 285 FORMAT(E15.9,5X, 'EMBEDUED COST OF PREFERRED')

475. C: *WRITE OUT BASE YEAR TOTAL ELECTRIC REVENUES

476. WRITE!WNN, 290) REVS

477. 290 FORMAT(E15.9,5X, 'ELECTRIC REVENBJE')

478. CKKHRITE OUT EASE YEAR NET INCOME

47\%. WRITE (NNN, 300) ENETIN

480. 300 FOFMAT(E15. $\%, 5 X_{1}$ 'NET INCOME')

481. COKRITE OUT BASE YEAR WDRKING CAPITAL

482. WRITE(NNN, 310) WC

483. 310 FOFMAT(E15.9,5X, 'WORKING CAPITAL')

484. C* WWRITE OUT BASE YEAR ACCUMULATED DEFERRED ITC

485. WRITE(NNN, 320) TDITC

486. 320 FORIAT (E15.9, 5', 'DEFERRED ITC')

487. C***WRITE OIT EASE YEAR ACCUMULATED DEFERREII INCOME TAXES

488. WRITE (NNN, 330) PRODEF

489. 330 FOSMAT (E15.9,5X, 'DEFERRED INCOME TAXES')

490. C*

491. WRITE(NNN, 340) TXFUEL

49\%. 340 FORMAT(E15.9,5X,'FUEL ADUUSTED FOR TAXES')

493. CK.KWRITE OUT BASE YEAR GENERAL TAX RATE

494. HRITE(NNM, 350) GTXRT

495. 350 FOFMAT(EI5, $3,5 X$, 'GENERAL TAX RATE')

496. RETURN

497. END

498. //60.FTOGF001 DO ISN=CNB922. PRI. FMIT773. DATA, DISO=(OLD, KEEP),

499. $/ / \quad V O L=S E R=F E 45 \%$, LNIT $=$ TAPES, LABEL $=1,,$, IN)

500. //GO.FTOFFOO1 DD *

501. 50 NUMEEF OF STATES

502. 142 STATE NUMBER, NCM REGION NLMBER

503. 20 IF STATE IS A SPLIT STATE (IE. MORE THAN! ONE REGION), 
504. 30 THEN ENTER ZERO.

505. $\quad 427$

506.544 .

507.60

508. 70

$509 . \quad 824$

$510 . \quad 92$

511. 1038

512. 1138

513. 120

514. 135

515. 140

516. 150

517. 1623

518. 179

519. 1810

520. 1919

521. 2018

522. 210

523. 2244

524. 231

525. 2438

526. 252

527. 268

528. 2717

529. 2842

$530 . \quad 2920$

531. $\quad 3021$

532. 3118

533. $\quad 3226$

534. $\quad 331$

535. $\quad 343$

$506 . \quad 3528$

537. 360

538. $\quad 374$

$539 . \quad 3816$

540. - 390

541. $\quad 4044$

542. $\quad 4129$

543. 420

544. 430

545. 442

546. 454

547. 4616

548. $\quad 470$

$54 \% . \quad 4843$

550. $\quad 4925$

551. 501

$552 . \quad 5137$

553. 520

$554 . \quad 5329$

555. 540

$556 . \quad 5511$

557. 5822

558. 214 NUMBER OF. ACCOUNTS TO BE ADUEII

55\%. 010021 ACCOUNT NIMRERS WHICH ARE TO BE AGGREGATED 
560. 010031 (ENTER CODES FOUND ON TAPE)

561. 010041

562. 010051

563. 010061

564. 010071

56.5. 010081

56.6. $0100 \% 1$

567. 010101

568. 010111

569. 010121

570. 010141

571. 010151

572. 010161

573. 010171

574. 010181

575. 010191

576. 010201

577. 010211

573. 010221

$579 . \quad 010231$

$580 . \quad 010241$

581. 010251

582. 010261

583. 010271

584. 010281

595. 010291

586. 010301

587. 010311

588. 010321

589. 010331

590.010341

591. 010351

532. 010361

593. 010371

594. 010381

595. 010391

596. 010401

597. 010411

5\%. 010421

$599 . \quad 010431$

600. 010441

601. $\quad 010451$

602. $0200 ! 1$

603. 020021

604. 020031

605. 020041

606. 020051

607. 020061

608. 020071

609. 020081

610. 020091

611. 020101

612. 020111

613. 020121

614. 020131

615. 020141 
616. 020151

617. 020161

618. 020171

619. 020181

620. 020191

621. 020201

622. 020211

623. 020221

624. 020231

625. 020241

626. 020251

627. 020261

628. 020271

629. 020281

630. 020291

631. 020301

632. 020311

633. 020321

634. 020331

635. $\quad 020341$

636. 020351

637. 020361

638. 020371

639. 020381

640. 020391

641. 020401

642. 030031

643. 030039

644. 040021

645. 040041

646. 040051

647. 040061

648. 040071

649. 040081

650. 040091

651. 040101

652. 040111

653. 040121

654. 040131

655. 040141

656. 040151

657. 040161

658. 040171

659. 040181

660. 040191

661. 040211

662. 040219

663. 040269

664. 040279

665. $\quad 040289$

666. 040299

667. 040309

668. $\quad 040319$

669. 040339

$670 . \quad 040349$

$671 . \quad 040359$ 
672. $\quad 040369$

673. 04033 ?

674. 040399

675. 040409

676. $\quad 040419$

677. 040429

678. 040439

67\%. 040449

$680 . \quad 040459$

681. 04046 \%

682. 040489

683. 040499

684. 040509

685. 040519

686. 040529

687. 040539

688. $\quad 040549$

689. 040569

$690 . \quad 040579$

691. 040589

692. 040607

69. 040619

694. 040629

695. 040639

696. 040649

697. $\quad 040659$

698. 041299

699. 041369

700. 070056

701. 070156

702. $\quad 070236$

703. $\quad 070326$

704. $\quad 070416$

705. 070426

706. $\quad 070536$

707. $\quad 070696$

708. $\quad 070836$

709. $\quad 070886$

710. 090102

711. $\quad 100051$

712. $\quad 100241$

713. $\quad 100621$

714. 110011

715. $\quad 110021$

716. $\quad 110031$

717. $\quad 110041$

718. $\quad 110051$

719. $\quad 110061$

720. $\quad 110071$

721. $\quad 110081$

722. $\quad 110091$

723. $\quad 110101$

724. $\quad 110111$

725. $\quad 120091$

726. $\quad 140021$

727. $\quad 140041$ 
728. 140051

729. $\quad 140001$

$730 . \quad 140071$

731. 140081

732. $\quad 1400 \% 1$

733. $\quad 140101$

734. $\quad 140121$

735. $\quad 140141$

736. $\quad 140151$

737. 140161

$730 . \quad 140171$

739. $\quad 140181$

740. $\quad 140191$

741. 140201

742. $\quad 140211$

743. $\quad 140221$

744. 140231

745. $\quad 140241$

746. $\quad 140251$

747. $\quad 140261$

$748 . \quad 140271$

749. $\quad 140281$

750. $\quad 140321$

751. $\quad 140331$

752. $\quad 140341$

753. $\quad 140351$

754. 140361

755. $\quad 140371$

756. 140331

757. $\quad 140391$

758. $\quad 140401$

759. 140421

760. $\quad 140431$

761. $\quad 140441$

762. 140451

763. 140461

764. $\quad 140471$

765. $\quad 140481$

766. $1404 \% 1$

767. $\quad 140501$

768. $\quad 140511$

769. 140521

$770 . \quad 140531$

771. 140541

$772 . \quad 140551$

773. 99 NO. OF EXEEPTILUN', IE. NO. OF UTILITIES SPLIT ACROSS NOM REGIONS

774. $011700 \quad 2 \quad 05.50 \quad 42.50$

775. $\quad 040540 \quad 3 \quad 27.56 \quad 23.04$ *UTILITY CODE, NUMBER OF REGIONS THAT

776. $\quad 050160 \quad 2 \quad 44.42 \quad 20.58$

*THE UTILITY SPLITS ACROSS, NCM REGION

777. $050220 \quad 1 \quad 441.0$

778. $061090 \quad 1 \quad 301.0$

779. $061240 \quad 1 \quad 311.0$

780. $061490 \quad 1 \quad 311.0$

781. $\quad 090450 \quad 2 \quad 01 \quad .13 \quad 02.07$

782. $\quad 110250 \quad 2 \quad 38.98 \quad 37.02$

783. $\quad 120290 \quad 1 \quad 061.0$ 
734. $\quad 12038002 \quad 06: 06 \quad 07.94$

785. $\quad 120560 \quad 1 \quad 071.0$

78t. $\quad 1206501$ ot 1.0

787. $\quad 121190 \quad 1 \quad 061.0$

788. $.170590 \quad 1 \quad 131.0$

789. $\quad \begin{array}{lllllll}180250 & 2 & 09 & .98 & 10.02\end{array}$

790. $\quad 180450 \quad 1 \quad 401.0$

791. $\quad \begin{array}{lllllll}180570 & 2 & 08 & .15 & 10 & .85\end{array}$

792. $\quad 190820 \quad 3 \quad 09.0519 .90 \quad 17.15$

793. $\quad 190890 \quad 1 \quad 191.0$

794. $\quad 190900 \quad 2 \quad 09.35 \quad 19.65$

795. $\quad 190970 \quad 2 \quad 19.99 \quad 16.01$

796. $\quad 200320 \quad 2 \quad 24.47 \quad 18.53$

797. $210850 \quad 1 \quad 121.0$

$\begin{array}{lllllll}\text { 798. } & 210910 & 2 & 12.28 & 13.72\end{array}$

799. $\quad 211270 \quad 1 \quad 13 \quad 1.0$

$800 . \quad 211900 \quad 1 \quad 131.0$

801. $\quad 220690 \quad 2 \quad 44.58 \quad 43.42$

802. $\quad \begin{array}{llllll}230600 & 2 & 01 & .32 & 02 & .68\end{array}$

803. $\quad \begin{array}{lllllll}230960 & 2 & 01 & .60 & 02 & .40\end{array}$

804. $\quad \begin{array}{lllllll}241050 & 3 & 38.72 & 37.13 & 35.15\end{array}$

$805 . \quad 241470 \quad 2 \quad 32.96 \quad 38.04$

806. $\quad \begin{array}{llllll}250270 & 2 & 01 & .03 & 02.97\end{array}$

807. $\quad 250570 \quad 1 \quad 021.0$

808. 250590 \& 021.0

$809 . \quad 250850 \quad 1 \quad 021.0$

810. $\quad 250960 \quad 2 \quad 01 \quad .02 \quad 02.98$

811. $\quad \begin{array}{llllll}251800 & 2 & 01 & .28 & 02 & .71\end{array}$

812. $\quad 26.0580 \quad 2 \quad 08 \quad .15 \quad 10.85$

813. $261310 \quad 1 \quad 081.0$

814. $271210 \quad 2 \quad 17.89 \quad 16.11$

815. $\quad \begin{array}{llllllll}290460 & 3 & 44.06 & 18.11 & 20 & .33\end{array}$

816. $\quad 290700 \quad 2 \quad 18.31 \quad 20.69$

817. $\quad 291500 \quad 3 \quad 09.17 \quad 19.07 \quad 20.76$

818. $301130 \quad 1 \quad 211.0$

819. $\quad 321460 \quad 2 \quad 30.14 \quad 26.36$

$820 . \quad 331230 \quad 1 \quad 01 \quad 1.0$

821. $360350 \quad 1 \quad 031.0$

822. $360400 \quad 1 \quad 331.0$

825. $360870 \quad 1 \quad 331.0$

824. 3610001031.0

825. 36105011031.0

326. $\quad 361150 \quad 2 \quad 03.98 \quad 33.02$

827. $361350 \quad 1 \quad 031.0$

828. 37036011041.0

82\%. $\quad 3706 \% 011041.0$

$\begin{array}{llllllll}830 . & 380800 & 3 & 21 & .28 & 16 & .62 & 22.10\end{array}$

831. $381150 \quad 2 \quad 17.47 \quad 16.53$

832. $390430 \quad 2 \quad 41.70 \quad 40.30$

833. $390470 \quad 1 \quad 391.0$

834. $390500 \quad 2 \quad 41.02 \quad 40.98$

835. $390560 \quad 1 \quad 401.0$

836. $\quad 391330 \quad 2 \quad 40.79 \quad 39.21$

837. $\quad \begin{array}{llllll}391370 & 2 & 41 & .23 & 40 & .67\end{array}$

838. $\quad 391410 \quad 2 \quad 41.23 \quad 40.67$

$839 . \quad 391470 \quad 1 \quad 401.0$ 


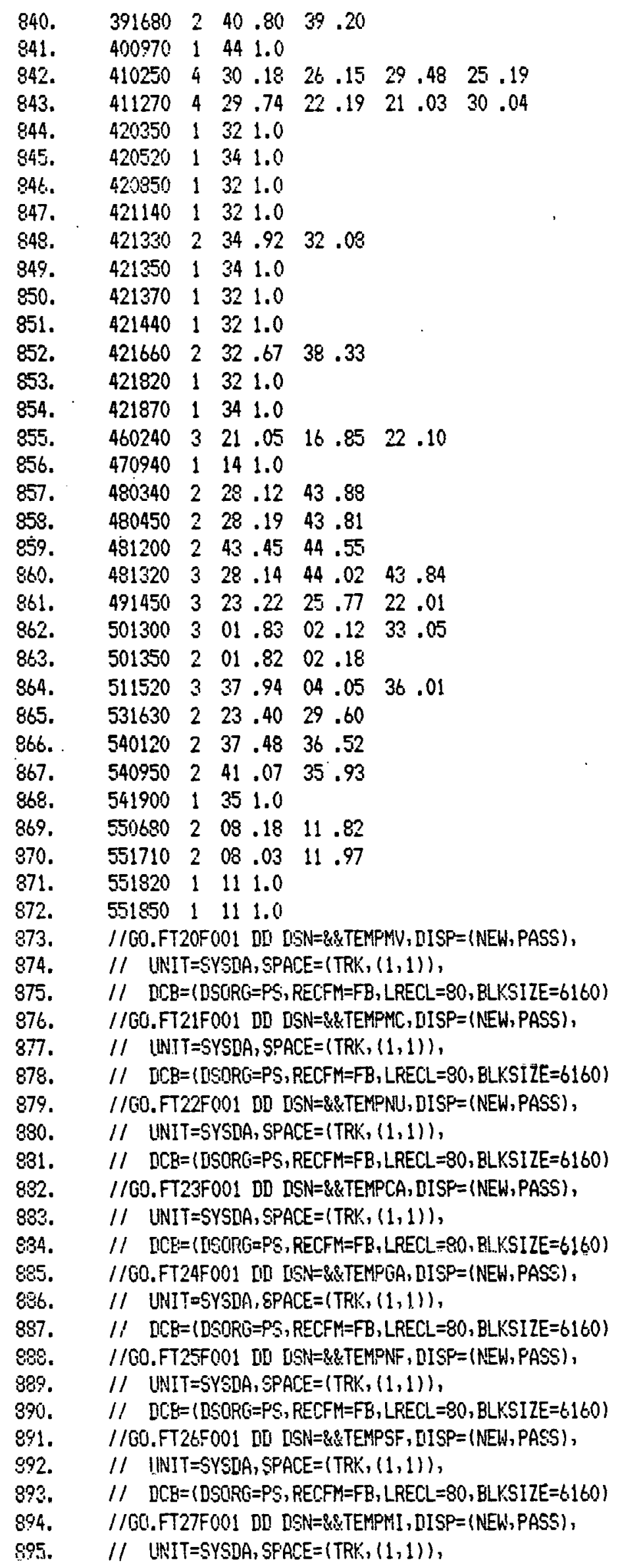


8\%6. I/ ICE (DSORG $=P S, R E C F H=F B, L R E C L=80$, BLKSIZE=6160)

87. //G0.FT2GF001 UD DSN=2\&TEMPIL, DISP=(NEW,PASS),

898. I/ UNIT=SYSDA, SPACE $=(T R K,(1,1))$,

89\%. I/ ICB $=($ ISOFL $6=P S, R E C F M=F B, L R E C L=80, B L K S I Z E=6160)$

900. //GO.FT29FOO1 DI DSH=?\&TEMPIN, IISF=(NEW,PASS),

901. I/ UNIT $=S Y S D A$, SPACE $=(T F K,(1,1))$,

902. I/ ICE =(DSORG =PS, RECFM=FB, LRECL $=80, B ! K S I Z E=6160)$

903. //60.FT30F001 DII ISN=\$\&TEMPHI, DISF=(NEW, PASS),

904. // UNIT $=S Y S D A, S P A C E=(T R K,(1,1))$,

905. $\quad / / \quad$ ICE $(D S O R G=P S$, RECFM $=F B, L R E C L=80$, BLKSIZE=6160)

906. //G0.FT31FO01 DU DSN=\&?TEMPEK, DISP=(NEW, PASS),

907. // UNIT=SYSDA, SFACE $=(T R K,\{1,1)\}$,

908. $/ / \quad D C B=(D S O R G=P S, R E C F Y=F B, L R E C L=80, B L K S I Z E=6160)$

909; $/ / G 0 . F T 32 F 001$ DO DSN=\&\&TEMPWK,DISP=(NEW, PASS),

910. // UNIT $=$ SYSDA, SPACE $=(\operatorname{TRK},(1,1))$,

911. I/ ICE=(DSORG=PS, RECFM=FB, LRECL $=80$, BLKSIZE $=6160$ )

912. //GO.FT33FOO1 OD DSN=\&\&TEMPET, DISP =(NEW, PASS),

913. I/ UNIT $=S Y S D A, S P A C E=(T R K,(1,1))$,

914. $/ 1 \quad D C E=(D S O R G=P S$, RECFM=FB, LRECL $=80$, BLKSIZE $=6160)$

915. //G0.FT34F001 DO [LSN=R\&TEMPUT, DISP=(NEH, PASS),

916. // LNIT $=S Y S D A, S P A C E=(T R K,(1,1))$,

917. I/ $D C E=(D S O R G=P S, R E C F M=F B, L R E C L=80, B L K S I Z E=6160)$

918. //G0. FT35F001 DD DSN=\&\&TEMPDK, DISP=(NEW, PASS),

919. I/ UNIT=SYSDA, SPACE $=(T R K,(1,1))$,

920. $\quad$ // $\quad$ DCB $=(D S O R G=P S, R E C F M=F B, L F E C L=80, B L K S I Z E=6160)$

921. //GO. FT36FOO1 DO DSNE\&STEMPMN, DISP=(NEW, PASS),

922. I/ UNIT $=$ SYSDA, SPACE $=\{\operatorname{TRK},(1,1)\}$,

923. $\quad / / \quad B C B=(D S O F C=P S$, RECFM $=F B, L R E C L=80$, BLKSIZE $=6160)$

924. //GO.FT37FOO1 DD DSN=\&\&TEMPKN, DISP=(NEU, PASS),

925. I/ UNIT $=$ SYSDA, SPACE $=(T T K,(1,1))$,

926. I/ IICE $=(I S O R G=P S, R E C F M=F B, L R E C L=80, E L K S I Z E=6160)$

S27. //GO. FT38F001 DO ISN=\&\&TEMPIA, DISP =(NEW, PASS),

928. // UNIT $T=S Y S D A, S P A C E=(T R K,(1,1))$,

929. // DCB $=([I S O R G=P S, R E C F M=F B, L R E C L=80, B L K S I Z E=6160)$

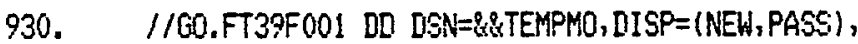

931. I/ UNIT $=$ SYSDA, SPACE $=(T R K,(1,1)\}$,

732. // DCE $=(I S O R G=F S$, RECFM $=F B, L R E C L=80, E L K S I Z E=6160)$

933. //GO.FT4OFOO1 DD DSN=\&\&TEMPMT, DISP=(NEW, PASS),

734. I/ UNIT $=$ SYSEA, SPACE $=($ TFK,$(1,1))$,

935. $/ / \quad$ DCE $=(0 S O R G=F S, R E C F M=F B, L R E C L=80, B L K S I Z E=6160)$

936. //GO.FT41FO01 DD DSN=\&\&TEMPWY, DISP=(NEW, PASS),

937. I/ UNIT=SYSDA, SPACE $=(\operatorname{TRK},(1,1))$,

939. I/ DCE $(D S O R S=P S, R E C F M=F E, \angle R E C L=80, B L K S I Z E=6160$ )

939. $/ / 60 . F T 42 F 001$ DII DSN=\&\&TEMPID, DISP $=(N E W, P A S S)$,

940. II UNIT $=$ SYSDA, SPACE $=(T R K,(1,1))$,

941. $/ / \quad U C B=(D S O R G=P S, R E C F M=F B, L R E C L=80, B L K S I Z E=6160)$

942. $/ / 60 . F T 43 F 001$ DD IISN=\&\&TEMPCO,DISP=(NEW, PASS),

943. $/ 1 \quad$ LNIT $=S Y S D A, S P A C E=(T R K,(1,1))$,

944. // ICEE=(IISGKG $=P S, R E C F M=F B, L R E C L=80$, BLLSIZE $=6160)$

945. //GO.FT44F001 DD ISN=\$\&TEMPU, DISP $=(N E W, P A S S)$,

946. // UNIT=SYSEA, SPACE $=(T R K,(1,1))$,

947. $/ / \quad D C B=(D S O R C=F S, R E C F M=F B, L R E C L=E 0, B L K S I Z E=6160)$

948. //GO.FT45F(OO1 DII DSV=\&\&TEMPAV, DISF=(NEW, PASS),

949. I/ UNIT $=$ SYSIA, SPACE $=(\operatorname{TRK},(1,1))$,

950. I/ LICE $(\mathrm{DSORG}=P S$, RECFM $=F B, L P E C L=80, B L K S I Z E=6160)$

951. //COU.FT46FOO1 IIII ISSN=\&:TEMPAZ, DISP=(NEW, PASS), 
952. I/ UNIT $=$ SYSLIA, SPACE $=(T R K,(1,1))$,

953. $/ /$ DCE $=$ (DSORL $=F S$, RECFM=FB, LRECL $=80$, BLKSIZE=6160)

954. //G0.FT47F001 DO DSN=\&\&TEMPNM. DISP=(NEH,PASS),

955. II UNIT $=$ SYSDA, SFACE $=(T R K,(1,1))$,

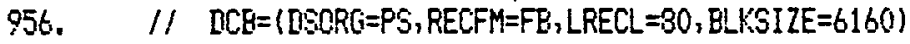

957. //GO.FT48FOO1 DO ISN=\&STEMFHO, DISP=(NEW, PASS),

958. I/ UNIT $=S Y S D A, S P A C E=(T R K,(1,1))$,

959. // IICE $($ ISORL $=P S$, RECFM=FB, LRECL $=80$, BLKSIZE=6160)

960. $\quad 1 / 60 . F T 49 F 001$ DD DSN=\&\&TEMPCN,DISF=(NEW, PASS),

961. I/ UNIT $=$ SYSDA, SPACE $=(T R K,(1,1))$,

962. $/ 1 \quad$ ICE $=\{$ ISORG $=F S, R E C F M=F B, \angle R E C L=80, B L Y S I Z E=6160)$

963. //GO.FT5OFO01 DD DSK $=\& \&$ TEMPCS, DISP=(NEW, PASS),

964. I/ UNIT $=S Y S D A$, SPACE $=(T R K,(1,1))$,

965. $\quad / 1$ DCB $=(D S D R C=P S, R E C F M=F B, L R E C L=80$, BLKSIZE $=6160)$

966. //G0.FT5IFO01 DO DSN=\&\&TEMPEP, DISP=(NEW,PASS),

967. I/ UNIT=SYSDA, SPACE $=(T R K,(1,1))$,

968. $/ 1 \quad$ ICB $=$ (DSORG $=P S$, RECFM=FB, LRECL $=80$, BLKSIZE $=6160$ )

969. $/ / 60 . F T 52 F 001$ DD DSN=\&\&TEMPJY, DISP $=$ (NEW, PASS),

970. I/ UNIT=SYSDA,SPACE $=\left\{T_{R K},(1,1)\right)$,

971. I/ $D C B=(D S O R G=P S, R E C F M=F B, L R E C L=80$, BLKSIZE=6160)

972. //GO.FT53F001 DO DSN=\&\&TEMFUP, DISP =(NEW, PASS),

973. I/ UNIT=SYSDA, SPACE $=(\operatorname{TRK},(1,1))$,

974. I/ DCE=(DSORG=PS, RECFM=FB, LRECL $=80$, BLKSIZE $=6160$ )

975. //G0.FT54F001 DD DSN=\&\&TEMPNH, DISF=(NEW, PASS),

976. I/ UNIT=SYSDA, SPACE $=(T R K,(1,1))$,

977. I/ DCE $=$ (ISORG $=P S, R E C F Y=F B, L R E C L=80$, ELKSIZE $=6160$ )

978. //G0.FT55F001 DII DSN=\&\&TEMPSW, DISP=(NEW, PASS),

979. $/ /$ UNIT=SYSDA, SPACE $=(\operatorname{TRK},(1,1))$,

980. I/ DCB $(D S O R G=P S, R E C F M=F B, L R E C L=80, B L K S I Z E=6160)$

981. $/ / G 0 . F T 56 F 001$ DD DSN=\&\&TEMPVA, DISF=(NEW, PASS),

982. I/ UNIT $=$ SYSDA, SPACE $=(T R K,(1,1))$,

983. I/ ICB $=(D S O R C=P S, R E C F M=F B, L R E C L=80, B L K S 1 Z E=6160)$

984. $/ / 60 . F T 57 F 001$ DN DSN=\&\&TEMPDM, DISF=(NEW, PASS),

985. I/ UNIT $=S Y S[A$, SPACE $=(T R K,(1,1))$,

996. I/ DCE $(D S O R C=P S, R E C F M=F B, \angle R E C L=80$, BLKSIZE $=6160)$

987. //G0.FT58FOO1 OD DSN=\&\&TEMPON, DISP=(NEW,PASS),

988. I/ UNIT $=$ SYSDA, SPACE $=\{T R K,(1,1)\}$,

939. I/ DCE $($ DSORG $=P S$, RECFM $=F B, \angle R E C L=80, B L K S I Z E=6160)$

Y90. //GO.FT59FOO1 DD DSN=LUTEMPON, DISP=(NEW, PASS),

991. II LINIT $=$ SYSDA, SPACE $=(\operatorname{TRK},(1,1))$,

992. I/ $D C B=(D S O R G=P S, R E C F Y=F B, L R E C L=80, B L K S I Z E=6160)$

993. //G0.FT6OFO01 DD DSN=\&\&TEMPOS,DISP=(NEW,PAES),

994. II UNIT=SYSDA, SPACE $=(T R K,(1,1))$,

995. I/ ICEF $=$ (ILSORG $=P 5$, RECFM=FE, LRECL $=80$, BLKSIZE $=6160)$

996. //GÜ.FT61FOO1 DD DSN=\&\&TEMPAM, DISP=(NEW,PASS),

997. I/ UNIT=SYSLA, SPACE $=(T R K,(1,1))$,

998. I/ DCB $=(D S O R G=P S, R E C F F=F B, L R E C L=80, B L K S I Z E=6160)$

999. $\quad / / 60 . F T 62 F 001$ DO DSN=\&\&TEMPTX,DISP=(NEW, PASS),

1000. I/ (NNIT $=$ SYSDA, SPACE $=($ TRK, $(1,1))$,

1001. I/ DCE $=(D S O R G=P S, R E C F M=F B, L R E C L=80, B L K S I Z E=6160)$

1002. //GO.FT63F001 DD DSR=\&\&TEMPAD, DISF=(NEW, PAGS),

1003. I/ LNIT $=$ SYSDA, SPACE $=\{T K K,(1,1))$,

1004. I/ [DCE $=(I S G G K G=P S, R E C F M=F B, L R E C L=80, E L K S I Z E=6160)$

1005. 1/60.FT64FOO1 DO IISK=\&\&TEMPNA, DISP=(NEW, PASS),

1006. $\quad / 1 \quad$ UNIT $=$ SYSDA, SPACE $=(\operatorname{TRK},(1,1))$,

1007. I/ DCE $=$ (DSORG $=F S, R E C F M=F B, L R E C L=80$, BLLSIZE $=\angle 160$ ) 
1008. I/DELD EXEC. PGM=IEFBR14, REGION=10K

100\%. //DD1 DD IISN=CNG376. MI2. NUREG. FORM1. PDS78, DISP=(MOD, DELETE),

1010. $/ 1$ UNIT $=$ DAGD, SPACE $=(T R K,(7,1,3))$

1011. //DELD EXEC. PGOY =IEFBR14, REGION=10K

1012. //LII DO IISN=CNE376. MI2. NUREG. FORM1. PDS78, UISP =(NEW, CATLG),

1013. I/ UNIT=DASI,

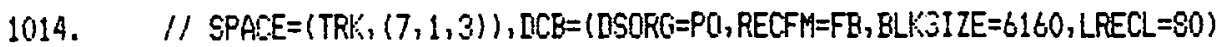

1015. //GENER EXEC. PGM=IEBGENER

1016. //SYSPRINT DEI DUMMY

1017. I/SYSUT1 DD DSN=\&\&TEMEMV, DISP $=$ (OLD, DELETE)

1018. //SYSUT2 DD LSN=CN6376. MI2. NUREG. FORM1. PDS78(MV), DISF=(OLD, KEEF)

101\%. //SYSIN DD DIMMY

1020. //GENER EXEC PGM=IEBGENER

1021. I/SYSPRINT DO DUMMY

1022. / /SYSUT1 DU DSN=\&\&TEMPMC, DISP =(OLD, DELETE)

1023. //SYSUT2 DI DSN=CN6376. MI2. NUREG. FORM1. PDS78(MC), DISP=(OLD, KEEP)

1024. I/SYSIN DO DUMMY

1025. //GENER EXEC PGM=IEBGENER

1026. //SYSPRINT DO DUMMY

1027. I/SYSUT1 DD DSN=\&\&TEMPNDI, DISP=(OLD, DELETE)

1028. //SYSUT2 DD DSN=CNG376.MI2. NUREG. FORM1. PLS78(NU), DISP=(OLD, KEEP)

1029. I/SYSIN DD DUMMY

1030. //GENER EXEC PGM=IEBGENER

1031. //SYSPRINT DI DIMMYY

1032. //SYSUT1 DD DSN=\&\&TEMPCA,DISP= (OLD, DELETE)

1033. //SYSUT2 DD DSN=CN6376. MI2. NUREG. FORM1. PDS78(CA), DISP=(OLD, KEEP)

1034. I/SYSIN DD DUUMY

1035. //GENER EXEC PGM=IEBGENER

1036. I/SYSPRINT DD DUMMY

1037. //SYSUTI OD USN=\&\&TEMPGA, DISP=(OLD, DELETE)

1038. //SYSUT2 DO ISN=CN6376.MI2. NUREG. FORM1. PDS78(GA), DISP=(OLD, KEEP)

103\%. I/SYSIN DD DUMMY

1040. //GENER EXEC PGM=IEBGENER

1041. //SYSPRINT DO DUMMY

1042. //SYSUT1 DII DSN=\&\&TEMPNF, OISP=(OLD, UELETE)

1043. //SYSUT2 DD IISN=CNG376. MI2. NUREG. FORM1. PDS78(NF), DISP=(OLD, KEEP)

1044. I/SYSIN DD LUMMY

1045. //GENER EXEC PGM=IEBGENER

1046. //SYSPRINT DO DUMAY

1047. //SYSUT1 DU DSN=\&\&TEMPSF, DISP=(OLD, DELETE)

1048. //SYSUT2 DD DSN=CN6376. MI2. NUREG. FORT1. PDS78(SF), DISP=(OLD, KEEF)

1049. I/SYSIN DD DUMMY

1050. //GENER EXEC. PGM=IEBGENER

1051. //SYSPRINT DD DUMMY

1052. - //SYSUT1 DO ISN=\&\&TEMPMI, DISP=\{OLD, DELETE)

1053. //SYSUT2 DD DSN=CNG376. MI2.NUREG. FORM1.PDS78(MI), IIISP=(OLD, KEEP)

1054. //SYSIN DD DUMAY

1055. //GENER EXEC PGM=IEBGENER

1056. //SYSPRINT DO IUMMY

1057. //SYSUT1 DO DSN=\&\&TEMPIL, DISP=(OLII, DELETE)

1058. //SYSUT2 DU ISN=CNG376. MI2. NUREG. FORM1.PIS78(IL), DISP=(OLD, KEEP)

105\%. //SYSIN DD DUMMY

1060. //GENER EXEC. PGY=IEBGENER

1061. //SYSPRINT DD DIMMYY

1062. I/SYSUT1 DO DSN=\&\&TEMPIN, DISP $=($ OLD, DELETE)

1063. //SYSUT2 DII DSN=CNE376. MI2. NUREG. FOFM1. PIS78(IN), DISP=\{OLD, KEEP) 
1064. I/SYSIN DD DUMYYY

1065. //GENER EXEC: PGY=IEEGENER

1066. //SYSPRINT DD DUMMY

1067. I/SYSUT1 DO DSN=\&\&TEMPWI, DISP=(OLD, DELETE)

1068. //SYSUT2 DD ISN=CNK376. MI2. NUREG. FORM1. PDS78(WI), DISP=(OLD, KEEP)

106\%. I/SYSIN ID DUMMY

i070. //GENER EXEC PGY=IEEGENER

1071. //SYSPRINT DO DUMMY

1072. //SYSUT1 DD ISN=\&\&TEMPEK, DISP=(OLD, DELETE)

1073. //SYSUT2 ID DSN=CN6376.MI2. NUREG. FORM1. PDS78(EK), IISP=(OLD, KEEF)

1074. I/SYSIN DD DUMMY

1075. I/GENER EXEC PGM=IEBGENER

1076. //SYSPRINT DD DUMMY

1077. //SYSUTI DD DSN=\&\&TEMPLK, DISP=10LD, DELETE)

1078. * //SYSUT2 DD DSNH=CN6376. MI2. NUREG. FORM1. PDS78(UK), DISP=(OLD, KEEP)

1079. I/SYSIN DD DIMAYY

1080. //GENER EXEC. PGM=IEBGENER

1081. //SYSPRINT DD DUMMY

1082. I/SYSUT1 DD DSN=\&\&TEMPET, DISP=(OLD, DELETE)

1083. I/SYSUT2 DD DSN=CN6376. MI2. NUREG. FORM1. PDS78(ET), DISF=(OLD, KEEP)

1084. //SYSIN DD DUMMY

1085. //GENER EXEC. PGM=IEBGENER

1085. //SYSPRINT DD DUMAY

1087. //SYSUT1 DN DSN=\&\&TEMPWT, DISP=(OLD, DELETE)

1083. I/SYSUT2 DD DSN=CNS376. MI2. NUREG. FORM1.PDS78 (WT), DISP=(OLD, KEEP)

1089. I/SYSIN DD DUMMY

1090. //GENER EXEC PGM=IEBGENER

1091. I/SYSPRINT DD DUMMY

1092. I/SYSUT1 DII DSN=\&\&TEMPDK, DISP=(OLD, DELETE)

1093. //SYSUT2 DO DSN=CNE376. MI2. NUREG. FORM1. PDS78(DK), DISP=(OLD, KEEP)

1094. //SYSIN DD DUMYY

1095. //GENER EXEC PGM=IERGENER

10?6. I/SYSPRINT DV DUMTY

1097. //SYSUT1 DO DSN=\&:TEMPMN, DISF=(OLD, DELETE)

1098. //SYSUT2 DD DSN=CNE376. MI2. NUREG. FORM1. PDS78(MN), DISP=(OLD, KEEP)

1099. I/SYSIN DI DUMMY

1100. I/GENER EXEC PGM=IEBGENER

1101. I/SYSPRINT DD DUMMY

1102. //SYSUT1 DO DSN=R\&TEMPKN, DISP=(OLU, DELETE)

1103. //SYSUT2 DD DSN=CN6376.MI2.NUREG. FORM1. PIS78(KN), DISP=(OLD, KEEP)

1104. I/SYSIN DO DUMMY

1105. //GENER EXEC PGM=IEBGENER

1106. I/SYSPRINT ID DUMMY

1107. //SYSUTI DN DSN=\&\&TEMPIA, DISP=(OLD, DELETE)

1108. //SYSUT2 DII ISN=CN6376. MI2. NURES. F(RM1.PDS78(IA), DISP=(OLD, KEEP)

1109. I/SYSIN OII DUMAY

1110. //GENER EXEC PGHFIEBGENER

1111. I/SYSPRINT DD DUMYY

1112. //SYSUT1 DD DSN=\&\&TEMPMO, DISF=(OLD, DELETE)

1113. I/SYSUT2 DD DSN=CN6376. MI2. NUREG.FORM1. PDS78(MO), DISP=(OLD, KEEP)

1114. I/SYSIN DD DUMPFY

1115. //GENER EXEC PGM=IERGENER

1116. //SYSPRINT IIII IUMMY

1117. //SYSUT! DO DSN=\&STEMPMT, DISP $=$ (OLD, DELETE)

1118. //SYSUT2 DD DSN=CN6376. MI2. NUREG. FORM1. PDS78 (MT), DISP=(OLD, KEEP)

1119. I/SYSIN DD DUMAY 
1120. //GENER EXEC PGH=IEBGENER

1121. //SYSPRINT DI DUMMY

1122. I/SYSUT1 DII DSN=\&\&TEMPWY, DISP=(OLD, DELETE)

1123. //SYSUT2 DO DSN=CN6376. MI2. NUREG. FORM1. PDS78(WY), DISP=(OLD, KEEP)

1124. //SYSIN DD DUMMY

1125. //GENER EXEC PGM=IEBGENER

1126. I/SYSPRINT DO DUMMY

1127. //SYSUT1 DUI DSN=\&\&TEMPID, DISP=(OLD, DELETE)

1128. //SYSUT2 DO DSN=CN6376. MI2. NUREG. FORM1. PDS78(ID), DISP=(OLD, KEEP)

112\%. //SYSIN DI IUMMY

1130. //GENER EXEC PGM=IEBGENER

1131. I/SYSPRINT DO DUMMY

1132. //SYSUT1 DII DSN=\&\&TEMPCO, DISP=(OLD, DELETE)

1133. //SYSIT2 DD DSN=CN6376. MI2. NUREG. FORM1.PDS78(CO), DISP=(OLD, KEEP)

1134. I/SYSIN DO DUMMY

1135. I/GENER EXEC PGM=IEEGENER

1136. I/SYSPRINT DD DUMYY

1137. //SYSUT1 DO DSN=\&\&TEMPUT, DISP=(OLD, DELETE)

1133. I/SYSUT2 DD DSN=CN6376.MI2. NUREG. FORM1.PDS78(UT), DISP=(OLD, KEEP)

1139. IISYSIN DD DUMMY

1140. //GENER EXEC PGH=IEBGENER

1141. I/SYSPRINT DD DUMYY

1142. //SYSUT1 DD DSN=\&\&TEMPN, DISP=(OLD, DELETE)

1143. //SYSUT2 DD DSN=CN6376. MI2. NUREG. FORM1. PDS78(NV), DISP=(OLD, KEEP)

1144. I/SYSIN DD DUMYY

1145. //GENEF EXEC PGM=IEBGENER

1146. I/SYSPRINT DD DUMMY

1147. I/SYSLT1 DD DSN=\&\&TEMPAZ, DISP= (OLD, DELETE)

1148. I/SYSUT2 DD DSN=CN6376. MI2. NUREG. FORM1.PDS78(AZ), DISP=(OLD, KEEP)

1149. I/SYSIN DO DUMYY

1150. //GENER EXEC. PGM=IEBGENER

1151. I/SYSFRINT DD DUMMY

1152. //SYSUT! DO DSN=\&\&TEMPNM, DISF=(OLD, DELETE)

1153. //SYSUT2 DD DSN=CN6378. MI2. NUREG. FORM1. PDS78(NM), DISP=(OLD, KEEP)

1154. I/SYSIN DD DUMMY

1155. //GENER EXEC PGM=IEBGENER

1156. //SYSPRINT DO DUMMY

1157. //SYSLII DD DSN=\&\&TEMPWO, DISP=(OLD, DELETE)

1158. //SYSUT2 DD DSN=CNG376. MI2. NUREG. FORM1. FEOTO(HO), DISP - (OLD, I(EEP)

1159. I/SYSIN DD DUMAY

1160. //GENER EXEC PGM=IEBGENER

1161. //SYSPRINT ID IUMMY

1162. //SYSUT1 DD DSN=\&\&TEYPCN, DISP=(OLD, DELETE)

1163. //SYSUT2 DO DSN=CNG376. MI2. AUREC. FORM1. PDS78(CN), DISP=(OLD, KEEP)

1164. I/SYSIN DD DUMYY

1165. I/GENER EXEC PGY=IEBGENER

1166. I/SYSPRINT DO DUMYY

1167. //SYSUT1 DI DSN=\&\&TEMPCS, DISF $=$ (OLD, LELETE)

1168. //SYSUT2 DD DSN=CNG376. MI2. NUREG. FORM1. PDS78(CS), DISP=(OLD, KEEP)

1169. I/SYSIN DD DUMYY

1170. //GENER EXEC PGH=IEBGENER

1171. I/SYSPRINT DD DLMYY

1172. //SYSUT1 DD DSN=\&\&TEMPEP, DISP= (OLD, DELETE)

1173. //SYSU2 DI DSN=CNE376. MI2. NUREG. FORM1. PDS78(EP), DISF=(OLD, KEEP)

1174. //SYSIN DO DUMMY

1175. //GENER EXEC PGM=IEBGENER 
1176. //SYSPRINT DO DUMAY

1177. I/SYSUT1 DN DSN=\&\&TEMFJY, DISP=(OLD, DELETE)

1178. I/SYSUT2 DD ISN=CN6376.MI2. NUREG. FORM1. PIS78(JY), DISP=(OLD, KEEP)

1179. //SYSIN DD DUMMY

1180. //GENER EXEC. PGM=IERGENER

1181. //SYSPRINT DO DUMMY

1182. //SYSUT1 DU DSN=\&\&TEMPUP, DISP=(OLD, DELETE)

1183. //SYSUT2 DD DSN=CNG374.M12. NUREG.FDRM1.PDS78(WP), DISP=(OLD, KEEP)

1184. //SYSIN DD CUMMYY

118.5. //GENER EXEC PGH=IEBGENER

1136. //SYSPRINT DD DUMMY

1187. //SYSIT1 DO DSN=\&\&TEMPNW, DISP=(OLD, DELETE)

1188. //SYSUT2 DD DSN=CN6376. MI2. NUREG. FORM1. PDS78(NW), DISP=(OLD, KEEP)

1189. I/SYSIN DD DAMMY

1190. / / GENER EXEC PGM=IEBGENER

1191. //SYSPRINT DD DUMYY

1192. //SYSUT1 DD DSN=\&\&TEAPSW, DISP =(OLD, DELETE)

1193. I/SYSUT2 DD DSN=CN6376.MI2. NUREG. FORM1.PDS78(SW), DISP=(OLD, KEEP)

1194. I/SYSIN DD DUPMY

1195. I/GENER EXEC PGM=IEBGENER

1196. I/SYSPRINT DO DUMMY

1197. //SYSUT1 DD DSN=\&\&TEMPVA, DISP=(OLD, DELETE)

1198. //SYSUT2 DD DSN=CN6376.MI2. NUREG. FORM1.PDS78(VA), DISP=(OLD, KEEP)

1199. I/SYSIN DD DUMMY

1200. I/GENER EXEC PGH=IEBGENER

1201. //SYSPRINT DD DUMMY

1202. I/SYSUT1 DD DSN=\&\&TEMPDM, DISP=(OLD, DELETE)

1203. //SYSUT2 DD DSN=CN6376.MI2. NUREG. FORM1. PDS78(DM), DISP=(OLN, KEEP)

1204. I/SYSIN DD DUMMY

1205. //GENER EXEC PGM=IEBGENER

1206. //SYSPRINT DO DUMANY

1207. I/SYSUT1 DD DSN=\&\&TEMPON, DISP=(OLD, IELETE)

1208. //SYSUT2 DU DSN=CN6376. MI2. NUREG. FORM1. PDS78(ON), DISP=(OLD, KEEP)

1209. //SYSIN DD DUMMY

1210. //GENEE EXEC PGM=IEBGENER

1211. //SYSPRINT DU DIUMYY

1212. //SYSUT1 DO DSN=\&\&TEMPOM, DISP=(OLD, DELETE)

1213. //SYSUT2 DD DSN=CN6376. MI2. NUREG. FORM1.PDS78(OM), DISP=(OLD, KEEP)

1214. I/SYSIN DD DUMMY

1215. //GENER EXEC. PGM=IEBGENER

1216. //SYSPRINT DD DUMMY

1217. - //SYSUT1 DO ISN $=\& \&$ \&EMPOS, DISP $=$ (OLD, DELETE)

1218. //SYSUT2 DD DSN=CN6376. MI2. NUREG. FORM1. PDS78(OS), DISP=(OLD, KEEP)

1219. I/SYSIN DD DUMMY

1220. //GENER EXEC PGM=IEBGENER

1221. //SYSPRINT DI MIMMY

1222. //SYSUT1 DD DSN=\&\&TEMPAM, DISP=(OLD, DELETE)

1223. //SYSUT2 DD DSN=CNK376. MI2. NUREG. F(RM1 . PD878(AM), DISPE(OLD, VEEEF)

1224. I/SYSIN DD DUMMY

1225. //GENER EXEC PGM=IEBGENER

1226. I/SYSPRINT DII DUMMY

1227. //SYSIT1 DO DSN=\&\&TEMPTX,DISP=(OLD, DELETE)

1228. //SYSUT2 DD DSN=CNS376. MI2. NUREG. FORM1.PDS78(TX), DISP=(OLD, KEEP)

122\%. I/SYSIN DD DIMMY

1230. //GENER EXEC PGM=IEBGENER

1231. //SYSPRINT DD DUMMY 
1232. (/SYSIT1 DO DSN=\&\&TEMPAO, DISP=(0! D, UELETE)

1233. //SYSUT2 DD DSN=CN6376.MI2. NUREG. FORY1. PDS78(AO), DISP=(OLD, KEEP)

1234. I/SYSIN DD DUMMY

1235. //GENER EXEC. PGM=IEBGENER

1236. I/SYSPRINT DII DUMMY

1237. (/SYSUT1 DO DSN=\&\&TEMFNA, DISP $=($ OLD, DELETE)

1238. //SYSUT2 DD IISN=CN6376.MI2. NUREG. FDFM1. PDS78(NA), [ISP=(OLD, KEEP)

123\%. //SYSIN UD [UIMMY 


\subsection{NUREG FORMIM.FORT}

This program calculates historical base year financial statistics for public utilities. It is very similar in structure to NUREG.FORMl. FORT. A listing now follows. 
1. //MI2UFORM JOB $\left(6376,[102,999),{ }^{\prime} F O F M 1 M^{\prime}\right.$, TIME $\left.=10,14\right)$, MSGCLASS=A

2. I/FPC EXEC FORTGCG, REGION=80OK

3. I/FORT.SYSIN DD *

4. IIIMENSION ISTATE(60), INFO(100), IEXCP(150), IREGS $(150,13)$,

5.

* PERCEN $(150,13)$, NOREG (150)

IOUULE PRECISION AMNT, AMNTI, AMOLNT $(45,100)$, AMAT2 $2(100)$

REAL $* 4$ ACODE, JTYF, KTYP, LTYP, MTYP, NTYP, OTYP, PTYF, OTYP,

* RTYF, FBRTTY, NOTYP, N1TYP, N2TYP, N3TYF, N4TYP, NSTYP, NSTYP,

* NTTYF, NETYP, NOTYF

DATA JTYP/'J'/

DATA KTYP/' $K^{\prime \prime} I$

DATA LTYP/'L'/

[ATA MTYP/'M'I

DATA NTYP/'N'I

DATA OTYP $/{ }^{\prime} \mathrm{O}^{\prime} /$

DATA PTYPI'P'I

DATA QTYP/'Q'I

18. DATA RTYP/'R'/
19. C DATA RBKTYP/'\}'/

20. IATA NOTYP $/{ }^{\prime} O^{\prime} /$

21. DATA NITYF/'1'/

22. DATA N2TYP/' $2 \prime 1$

23. DATA N3TYP $/ 3$ ' $/ 1$

24. DATA N4TYP/'4'I

25. DATA NSTYP/ $/ 5 / 1$

26. DATA NGTYP/' 6 'l

27. [ATA NTTYP/'7'I

28. DATA NBTYP/'8'I

29. [IATA NOTYP/' $/ 91$

30. C* NNIREG. FORMIM. FORT. THIS PROGRAM READS DATA FROM THE FORM IM TAPE AND

31. C. WRITES OUT DATA NEEUEI FOR THE NATIONAL UTILITY REGULATORY

32. C $*$ H MODEL (NUREGi).

33. CKस

34. C** HRITTEN EY MAFK R. INGLIS, ICF INC. DEC 31,1980

35. $\quad$ C*

36. C*

37. CHAANII ACCCUNT CODES.

38. C C NETATE $=\#$ OF STATES

39. C NINFO= \# OF ACCOUNTS TO BE ADDDED OVER REGIONS

40. C ISTATE(I)=DOE REGION THAT STATE I IS IN

41. C INFO(I) $=6$ DIGIT ACCOUNT CODE

42. C NEXCP=\# OF UTILITITIES WHICH ARE SPLIT ACROSS REGIONS

43. COR ARE FEDERAL PROJECTS.

44. C IEXCP $=$ ID \# OF SPLIT UTILITY

45. C. COREC $=$ OF NCM REGIONS WHICH UTILITY SPLITS ACROSS

46. C (IREGS(I,J), JII, NOREG(I)) = THE CODES OF THE REGIONS WHICH

47. $\quad$ SPLIT THE UTILITY.

48. C* (PERCEN $(I, J), J=1$, NOREG(I)) = THE \% SPLITS CORRESPONDING TO

49. C IREGS REGIONS.

50. READ (9,*)NSTATE

51. DO 77 J $=1$, NSTATE

52. 77 READ $(9, *)$ I,ISTATE(I)

53. READ $(9, *)$ NINFO

54. IIO $88 I=1$, NINFO

55. 88 READ $(9,802)$ INFO(I) 


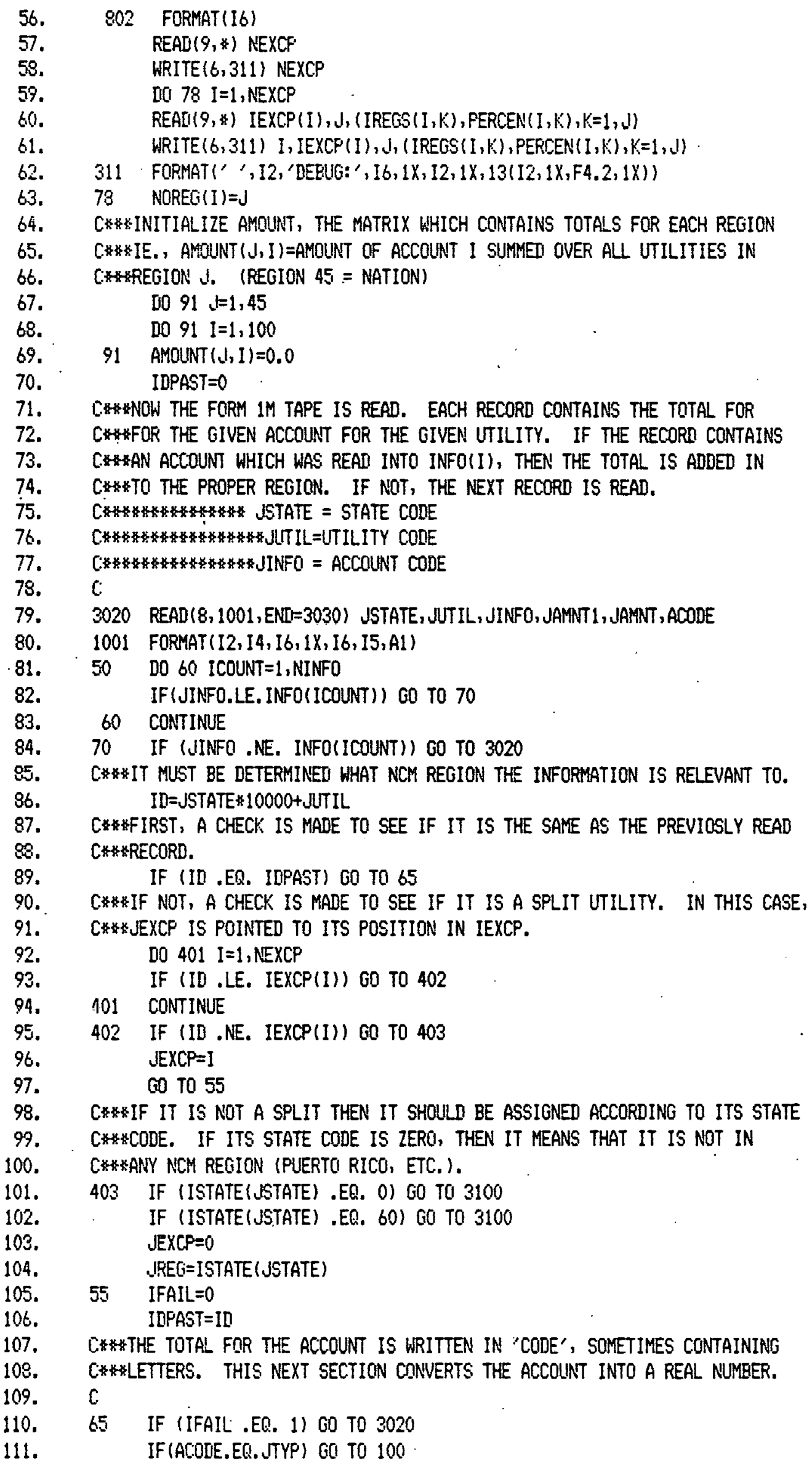




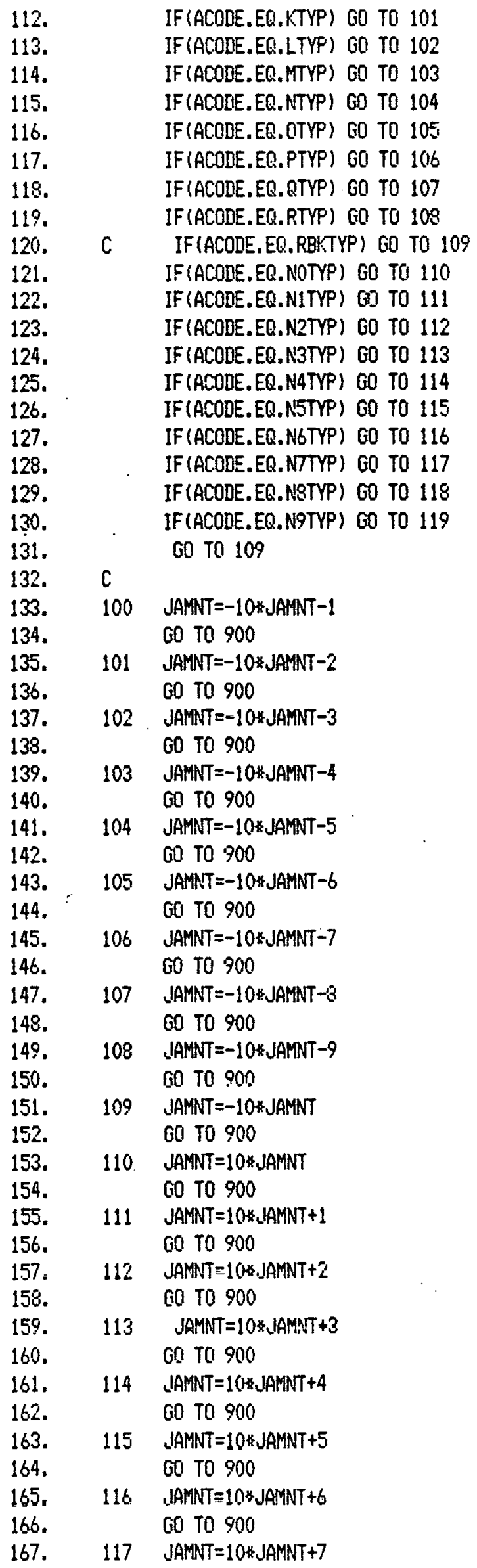




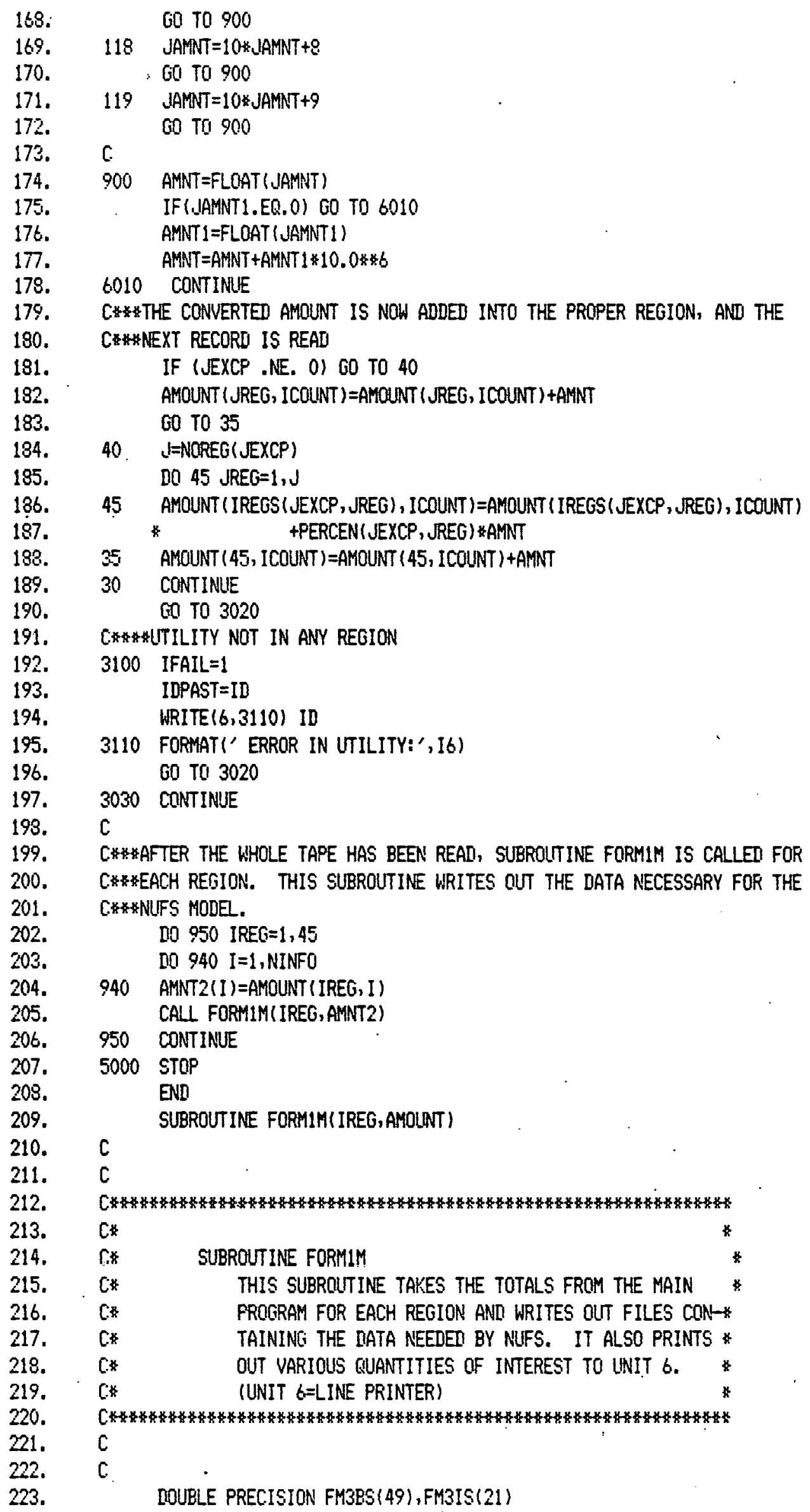


224. DOUBLE PRECISION FM3EPS(17), FM3DEP(3)

225. DOURLE PRECISION TEMP5, TEMP6, EPPOR, AMOUNT(100)

226. DIMENSION ZAI(10)

227. C**FIRST, PUT IATA INTO MATRICES BY SCHEDUE:

228. C FMBES=BALANCE SHEET

22\%. F $*$ FMIIS=INCOME SHEET

230. CW FMEPS=ELECTRIC PLANT IN SERVICE

231. C* ETC.

232. ICOUNT $=0$

233. $\quad$ ए0 $12 \quad I=1,49$

234. ICOUNT $=$ ICOUNT +1

235. 12 FM3BS(I)=AMOUNT (ICOUNT) $/ 1000000$.

236. $\quad$ DO $20 \quad I=1,21$

$237 . \quad$ ICOUNT $=$ ICOUNT +1

238. $20 \quad$ FM3IS(I) $=$ AMOUNT (ICOUNT) $/ 1000000$.

23\%. ICOLNT $=$ ICOUNT +1

240.

241.

242.

243.

244.

245.

246.

247.

248.

249.

250.

251

252

SALES=AMOUNT (ICOUNT)/1.0E09

DO $30 \mathrm{I}=1,17$

ICOUNT $=$ ICOLNT +1

30 FM3EPS(I) =AMOUNT (ICOUNT) $/ 1000000$.

I0 $40 \quad I=1,3$

ICOUNT $=$ I COUNT +1

40 FM3DEP (I) =AMOUNT (ICOUNT $/ 1000000$. )

GENER=AMOUNT (ICOUNT+1)/1.0EO9

C.

WRITE $(6,11)$ IREG

11 FORMATI' THE TOTALS FOR REGION ', I2,':?)

c

253.

254.

255.

256.

257.

258.

259.

260.

261.

262.

263.

264.

265.

266.

267.

268.

$26 \%$.

270.

271.

272.

273.

274.

C* *NOW STRIP OFF RELEVANT AMOUNTS FOR NUFS AND INTEREST. (IE. THIS

C*ROUTINE CAN BE MODIFIED TO PRINT OUT ANY ACCOINT.)

C

WRITE $(6,311)$

311 FORMAT!' THE VALUES FOR FM3EPS ARE:'>

DO $9011 \mathrm{~J}=1,17$

9011 WRITE $(6,2015)$ FMJEPS(J)

2015 FORMAT $(5 X, F 10.2)$

$Z A 1(1)=F M 3 E P S(1)$

ZAI $(2)=$ FM3EPS(2)

$2 A 1(3)=F M 3 E P S(3)$

$Z A 1(4)=$ FM3EPS $(4)$

$Z A 1(5)=0.0$

$Z A 1(6)=F M 3 E P S(5)$

$2 A 1(7)=$ FM3EPS (7)

ZA! $(8)=$ FM3EPS $(8)$

$Z R 1(9)=F M 3 E P S(9)$

$2 A 1(10)=0.0$

WRITE $(6,2014)$

2014 FORMATI' THE VALUES FOR IA(I) ARE')

DO $9015 \mathrm{~J}=1,10$

9015 WRITE $(6,2015)$ ZAI (J)

IF (FM3EPS(6),EQ.0.QR. FM3EPS(10).EQ.0)GO TO 100:)

275. $\quad C$

276. FINI RELATIVE ERROR FOR ASSET TOTALS.

277. C $\quad$ RE1-RE2 = RELATIVE ERRORS

278. C

279.

TEMP5 $=0.0$ 


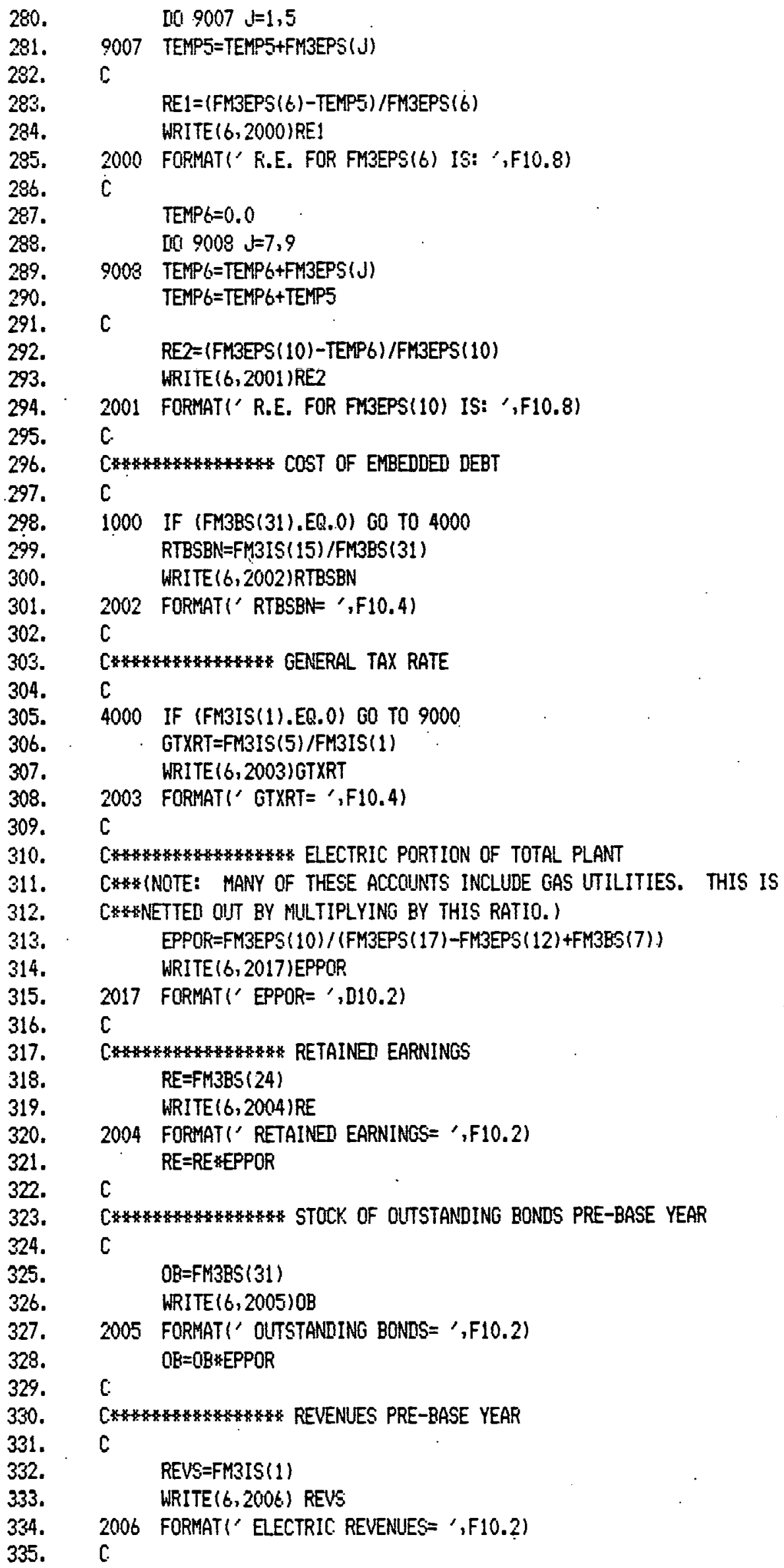


336. Cस* C

337.

338.

C

339.

340.

341.

342.

343.

344.

345.

346.

347.

348.

349.

350.

351.

352.

353.

354.

355.

356 .

357.

358.

359.

360.

361.

362.

363.

364.

365.

366.

367.

368.

$36 \%$.

370.

371.

372.

373.

374.

375.

376.

377.

378.

379.

380.

331.

382.

38.

384.

385.

386.

397.

388.

339.

390.

391.

$T E M P 7=F M 3 B S(25)+F M 3 B S(31)+F M 3 B S(33)$

$W 1=F M 38 S(31) /$ TEMP7

WRITE(6, 2007) WI

2007 FORMAT(' $W 1=', F 10.4)$

H2=FM3ES(33)/TEMP7

WRITE $(6,2008)$ W2

2008 FORMAT(' W2 $=$ ',F10.4)

W3=FM3BS(25)/TEMP7

WRITE $(6,2009)$ W3

2009 FORMATI' $W 3=$ ',F10.4)

$W 4=0.0$

WRITE $(6,2010) \quad 44$

2010 FORMATI' W4= ',F10.4)

C.

C*** FU \& O\&M COSTS ADUULTED FOR TAXES TXFUEL $=(F M 3 I S(2)+F M 3 I S(3))$

WRITE(6, 2013) TXFUEL

2013 FORMATI' FUEL PLUS O\& $M=', F 10.21$

TXFUEL=TXFUEL/(1-GTXRT)

C.

C*** WORKING CAPITAL

C

WC $=(F M 3 B S(15)-F M 3 B S(39))$

WRITE(6,2021) WC

2021 FORMATI' HORKING CAPITAL= ',F10.41

WC $=W C \times E P P O R$

C

C* COMMON STOCK PRE-BASE YEAR

C

COMSTK $=($ FM3BS (25) -FM3BS(24))

WRITE $(6,2022)$ CONSTK'

2022 FOFMATI' COMMON STOCK $=', F 10.4)$

COMSTK $=$ COMSTK $*$ EPPOR

c.

C* $*$ CONSTRUCTION WORK IN PROGRESS ACCOLNT

C

WRITE (6, 2025) FM3EPS(12)

2025 FORMATI' CWIP ACCOUNT: ',F10.2,'1')

C.

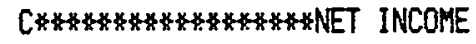

C

ENETIN=FM3IS(21)

WRITE $(6,2028)$ ENETIN

2028 FOFMATI' NET INCOME $\left.={ }^{\prime}, F 10.4\right)$

C $* *$

C**ANOW WRITE RESULTS TO FILES FOR NUFS.

C***

9000 NNNE=IREG+19

C* WWITE OUT BASE YEAR GENERATION

WRITE (NAN, 200) GENER

200 FORMAT (E15.9,5\%, 'GENERATION')

WRITE $(6,200)$ GENER

C***WRITE OUT BASE YEAR SALES

WRITE(NNN, 210) SALES 
392. 210 FORMAT(E15.9,5X,'SALES TO ULTIMATE')

393. WRITE $(6,210)$ SALES

394. . C* KRRITE OUT EXISTING ASSET BASE

395. WRITE(NNN, 220) (ZAI (I), $]=1,10)$

396. 220 FIRMAT(E15.9,5X,'INTANGIBLE (EXISTING)'/E15.9,5X,'STEAM PROEU'

397. 1 'CTION'/E15.9,5X, 'NUCLEAR PRONUCTION'/E15,9,5X, 'HYDRO CONN'

398. 2 E15.9.5X,'PUMPED STORAGE'/E15.9,5X,'OTHER PRODUCTION'/E15.9,

$399 . \quad 3$ 5X, 'TRANSMISSION'/E15.9,5X, 'DISTRIEUTION'/E15.9, 5X, 'GENERAL'/

400. 4 E15.9,5X, 'COMMON-ELEC')

401. CWHRITE OUT BASE YEAR STOCK OF BONDS

402. WRITEINNN, 230) OB

403. 230 FORMAT (E15.9,5X, 'OUTSTANDING BONDS')

404. CB\#WRITE OUT BASE YEAR COMMON STOCK

405. WRITE (NNN, 250) COMSTK

406. 250 FORMAT(E15.9,5X,'COMMON STOCK')

407. CHURITE OUT BASE YEAR RETAINED EARNINGS

408. WRITE (NNN, 270) RE

409. $270^{\circ}$ FORMAT(E15.9,5X,'RETAINED EARNINGS')

410. CWURITE OUT BASE YEAR EMBEDDED COST OF LONG TERM DEBT

411. WRITE(NNN, 280) RTESBN

412. 280 FORMAT (E15.9,5X,'COST OF EMBEDDED DERT')

413. C**WRITE OUT BASE YEAR TOTAL ELECTRIC REVENUES

414. WRITE(NNN, 290) REVS

415. 290 FORMAT (E15,9,5X, 'ELECTRIC REVENUES')

416. CWITE OUT BASE YEAR NET INCOME

417. WRITE(NNW, 300) ENETIN

418. 300 FORMAT(E15.9,5X, NET INCOME')

419. C*HRITE OUT BASE YEAR WORKINO CAPITAL

420. WRITE(NNN, 310) HC

421. 310 FQRMAT(E15.9,5X, 'WORKING CAPITAL')

422. C*HRITE OUT BASE YEAR FUEL AND ORA COSTS ADUUSTED FOR TAXES

423. WRITE(NNN, 340) TXFUEL

424. 340 FORMAT(E15.9,5X,'FUEL ADJUSTED FOR TAXES')

425. CWURITE OUT EASE YEAR GENERAL TAX RATE

426. WRITEINNN, 350) GTXRT

427. 350 FORMAT(E15.9,5X,'GENERRL TAX RATE')

428. RETLRN

429. END

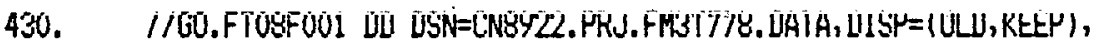

431. $/ / \quad V O L=S E R=F 14225$, UNIT TAPES, LABEL $=(,,$, IN)

432. //GO.FTOFFO01 DD *

433. 56 NUMBER OF STATES

434. 142 STATE NUMBER, NCM REGION NUMBER

435. 20 IF STATE IS A SPLIT STATE (IE. MORE THAN ONE REGION),

436. 30 THEN ENTER ZERO.

437. $\quad 427$

438.544

439. 60

440. 70

441. 824

442. 92

443. 1038

444. 1138

445. 120

446. 135

447. 140 
448. $\quad 150$

449. 1623

450. 179

451. $\quad 1810$

452. 1919

453. $\quad 2018$

454. 210

455. 2244

45t. 231

457. $\quad 2438$

458. $\quad 252$

459. 268

460. 2717

46!. $\quad 2842$

462. 2920

463. 3021

464. $\quad 31.18$

465. $\quad 3226$

466. $\quad 331$

467. $\quad 3433$

468. $\quad 3528$

469. 360

470. $\quad 374$

471. 3816

472. 390

473. $\quad 4044$

474. $\quad 4129$

475. $\quad 420$

476. $\quad 430$

477. $\quad 442$

478. $\quad 454$

479. $\quad 4616$

$480 . \quad 470$

481. $\quad 4843$

482. $\quad 4925$

483. 501

484. 5137

485. $\quad 520$

436. $\quad 5329$

487. 540

488. 5511

489. 5622

490. $\quad 92$

NUMBER OF ACCOUNTS

491. 010021 ACCOUNT NUMBERS WHICH ARE TO BE AGGREGATED

492. 010041 (ENTER CODES FOUND ON TAPE)

493. $\quad 010051$

494. 010091

495. 010101

496. 010111

497. 010121

498. 010141

499. 010151

500. 010181

501. 010191

502. 010201 
503. 010211

504. 010221

505. 010231

506. 010251

507. 010261

508. 010271

509. 010291

510.010301

511. 010371

512. 020021

513. 020031

514. 020041

515. 020051

516. 020071

517. 020081

518. 020091

519. 020101

520. 020111

521. 020121

522. 020141

523. 020151

524. 020161

525. 020171

526. 020181

527. 020191

528. 020201

529. 020211

530. 020231

531. 020241

532. 020251

533. 020261

534. 020281

535. 020291

536. 020301

537. 020311

533. 020321

$539 . \quad 020371$

540. 030011

541. $\quad 030021$

542. 030031

543. 030041

544. 030051

545. 030061

546. 030071

547. 030081

540. 030091

549. 030101

$550 . \quad 030121$

551. . 030131

552. 030141

553. 030151

554. 030161

555. 030171

556. 030181

557. 030191

558. 030201 
55\%. $\quad 030211$

560. 030221

561. $\quad 040462$

562. 060011

563. 060021

564. 060031

565. 060041

566. 060051

567. 060061

568. 060071

569. 060081

570. 060091

571. 060101

572. 060111

573. 060121

57. 060131

575. 060141

576. 060151

577. 060161

578. 060181

579. 070211

580. 070221

581. 070251

582. 090051

583. 126 NO. OF EXCEPTIONS, IE. NO. OF UTILITIES WHICH SPLIT ACROSS NCM REGIONS

584. 06510011301.0 \#UTILITY CODE, NIMBER OF NCM REGIONS WHICH THE

585. 065170 1 31 1.0 *UTILITY SPLITS ACROSS, NCM REGION NUMBER,

586. $065240 \quad 131 \quad 1.0$ *PERCENTAGE ALLOTTED TO REGION

587. $065450 \quad 1 \quad 31 \quad 1.0$

583. $065730 \quad 1 \quad 31 \quad 1.0$

599. $065900 \quad 1 \quad 31 \quad 1.0$

590. $065940130 \quad 1.0$

591. $066080 \quad 1 \quad 31 \quad 1.0$

592. $0666170 \quad 1 \quad 30 \quad 1.0$

593. $066220 \quad 1 \quad 30 \quad 1.0$

594. $0662901131 \quad 1.0$

595. $0663201130 \quad 1.0$

596. $0664301131 \quad 1.0$

597. $0665201130 \quad 1.0$

$5 \%$. $\quad 0665501130 \quad 1.0$

599. $\quad 066570 \quad 1 \quad 30 \quad 1.0$

600. 06659011301.0

601. $067000 \quad 1 \quad 30 \quad 1.0$

602. $067860130 \quad 1.0$

603. $\quad 125410 \quad 1 \quad 07 \quad 1.0$

604. $\quad 1254701107 \quad 1.0$

605. $\quad 125600 \quad 1 \quad 07 \quad 1.0$

606. $125900107 \quad 1.0$

607. $\quad 125970 \quad 1$ of 1.0

608. $\quad 126030 \quad 1 \quad 06 \quad 1.0$

6.09. $\quad 126340 \quad 1 \quad 06 \quad 1.0$

610. $\quad 126400 \quad 1 \quad 06 \quad 1.0$

611. $\quad 126890 \quad 1 \quad 06 \quad 1.0$

612. $1269501107 \quad 1.0$

613. $215470 \quad 1 \quad 13 \quad 1.0$

614. $215690 \quad 1 \quad 13 \quad 1.0$ 


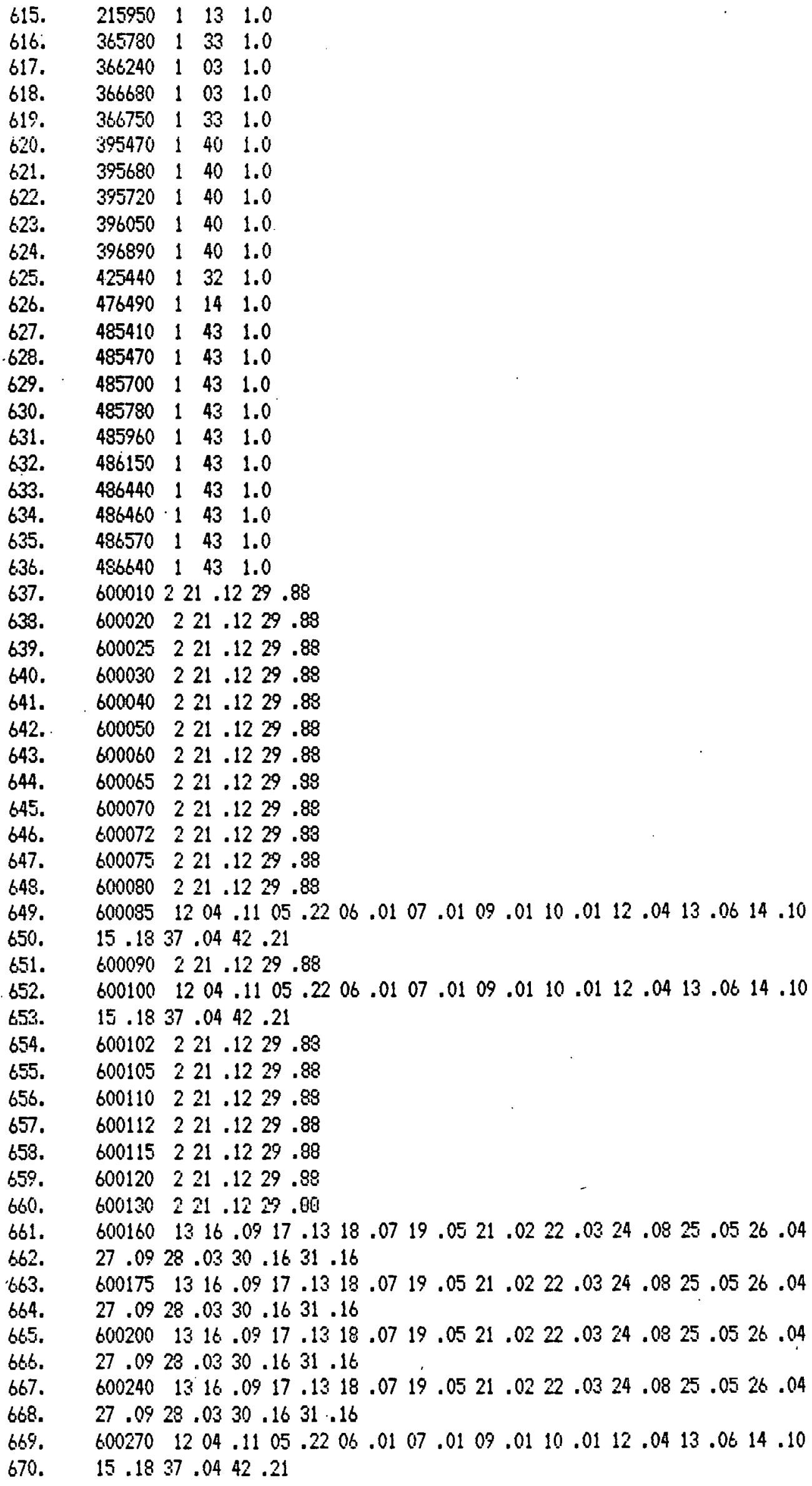


671. $\quad 600280 \quad 1204.1105 .2206 .01 \quad 07 \quad .0109 .01 \quad 10.01 \quad 12 \quad .04 \quad 13 \quad .06 \quad 14 \quad .10$

672. $\quad 15.1837 .0442 .21$

673. $\quad 600285 \quad 1204.11 \quad 05.2206 .01 \quad 07 \quad 0109.01 \quad 10.01 \quad 12.04 \quad 13.06 \quad 14 \quad .10$

674. $\quad 15.1837 .0442 .21$

675. $\quad 600290 \quad 1204.11 \quad 05.2206 .01 \quad 07.0109 .01 \quad 10.01 \quad 12.04 \quad 13.06 \quad 14 \quad .10$

676. $\quad 15.1837 .0442 .21$

677. $\quad 600300 \quad 1204.1105 .2206 .0107 .0109 .01 \quad 10.01 \quad 12.04 \quad 13.06 \quad 14.10$

675. $\quad 15.1837 .0442 .21$

679. $\quad 600310 \quad 1204.1105 .2206 .01 \quad 07 \quad 0109.01 \quad 10.01 \quad 12.04 \quad 13.06 \quad 14 \quad 10$

680. $\quad 15.1837 .0442 .21$

681. $\quad 600320 \quad 1204.1105 .2206 \quad 01 \quad 07 \quad 0109.01 \quad 10.01 \quad 12 \quad .04 \quad 13 \quad .06 \quad 14 \quad .10$

682. $\quad 15.1837 .0442 .21$

683. $\quad 600325 \quad 1204 \quad 1105.2206 .0107 .0109 .01 \quad 10.01 \quad 12.04 \quad 13.06 \quad 14.10$

684. $\quad 15.1837 .0442 .21$

685. $600330 \quad 1204 \quad .1105 .2206 .0107 .0109 .01 \quad 10: 01 \quad 12.04 \quad 13 \quad .06 \quad 14.10$

686. $\quad 15.1837 .0442 .21$

687. $\quad 600340 \quad 1204.11 \quad 05.2206 .01 \quad 07 \quad 0109.01 \quad 10.01 \quad 12 \quad .04 \quad 13 \quad .06 \quad 14 \quad 10$

689. $\quad 15.1837 .0442 .21$

689. $\quad 600350 \quad 1204 \quad .11 \quad 05.2206 .01 \quad 07.0109 .01 \quad 10.01 \quad 12 \quad .04 \quad 13 \quad .06 \quad 14 \quad 10$

690. $\quad 15.1837 .0442 .21$

691. $\quad 600360 \quad 1204.11 \quad 05.2206 .01 \quad 07 \quad .0109 .01 \quad 10.01 \quad 12 \quad .04 \quad 13.06 \quad 14.10$

692. $\quad 15.1837 .0442 .21$

693. $\quad 600365 \quad 1204.11 \quad 05.2206 .01 \quad 07.0109 .01 \quad 10.01 \quad 12.04 \quad 13.06 \quad 14 \quad 10$

694. $\quad 15.1837 .0442 .21$

6.95. $\quad 600370 \quad 1204.1105 .2206 .01 \quad 07.0109 .01 \quad 10.01 \quad 12.04 \quad 13.06 \quad 14 \quad .10$

696. $\quad 15.1837 .0442 .21$

697. $\quad 600380 \quad 1204.11 \quad 05.2206 .01 \quad 07 \quad .0109 .01 \quad 10.01 \quad 12.04 \quad 13.06 \quad 14 \quad .10$

698. $\quad 15.1837 .0442 .21$

699. $\quad 600385 \quad 1204 \quad .11 \quad 05.2206 .01 \quad 07.0109 .01 \quad 10.01 \quad 12 \quad .04 \quad 13.06 \quad 14 \quad .10$

700. $\quad 15.18 .37 .0442 .21$

701. $\quad 600390 \quad 1204.11 \quad 05.2206 .01 \quad 07.0109 .01 \quad 10.01 \quad 12.04 \quad 13.06 \quad 14 \quad .10$

702. $\quad 15.1837 .0442 .21$

703. $\quad 600395 \quad 1204 \quad .1105 .2206 .01 \quad 07.0109 .01 \quad 10.01 \quad 12 \quad .04 \quad 13 \quad .06 \quad 14 \quad .10$

704. $\quad 15.1837 .0442 .21$

705. $\quad 600400 \quad 1204.11 \quad 05.2206 .01 \quad 07.0109 .01 \quad 10.01 \quad 12 \quad .04 \quad 13.06 \quad 14 \quad .10$

706. $\quad 15.1837 .0442 .21$

707. 600410418.0120 .3743 .0844 .54

708. $\quad 600415 \quad 418.0120 .3743 .0844 .54$

709. $\quad 600420 \quad 4 \quad 18.0120 .3743 .0844 .54$

710. $\quad 600425 \quad 418.0120 .3743 .0844 .54$

711. $600430 \quad 418.0120 .3743 .0844 .54$

712. $\quad 600435 \quad 4 \quad 18.0120 .3743 .0844 .54$

713. $\quad 600436 \quad 418.0120 .3743 .0844 .54$

714. $\quad 600440 \quad 418.0120 .3743 .0844 .54$

715. $\quad 600445 \quad 418.0120 .3743 .0844 .54$

716. $\quad 600450 \quad 418.0120 .3743 .0844 .54$

717. $600460 \quad 418.0120 .3743 .0844 .54$

718. $\quad 600461 \quad 4 \quad 18.0120 .3743 .0844 .54$

719. $\quad 600462 \quad 1204.1105 .2206 .01 \quad 07.0109 .01 \quad 10.01 \quad 12.04 \quad 13.06 \quad 14 \quad .10$

720. $\quad 15.1837 .0442 .21$

721. $\quad 600465 \quad 418.0120 .3743 .0844 .54$

722. $\quad 600470 \quad 418.0120 .3743 .0844 .54$

723. $\quad 600480 \quad 418.0120 .3743 .0844 .54$

724. $\quad 600483418.0120 .3743 .0844 .54$

725. $600485 \quad 418.0120 .3743 .0844 .54$

726. $600483 \quad 418.0120 .3743 .0844 .54$ 
727. $\quad 6.00490 \quad 4 \quad 18.0120 .3743 .0844 .54$

728. $600500 \quad 418.0120 .3743 .0844 .54$

729. $\quad 600505 \quad 418.0120 .3743 .0844 .54$

730. $\quad 600510 \quad 4 \quad 18.0120 .3743 .0844 .54$

731. $\quad 600530 \quad 3 \quad 31.45 \quad 26.27 \quad 27.28$

732. $\quad 600540 \quad 221.1229 .88$

733. $\quad 600550 \quad 1316.0917 .1318 .0719 .0521 .0222 .0324 .0825 \quad 0526.04$

734. $\quad 27.0928 .0330 .1631 .16$

735. $600576 \quad 1081.0$

736. $\quad 600580605.0212 .0213 .0614 .2315 .4142 .26$

737. //GO.FT2OF001 DD DSN=\&\&TEMPNV, DISP=(NEW,PASS),

738. II UNIT=SYSDA; SPACE $=(T R K,(1,1))$,

739. II DCB $=$ (DSORC=PS, RECFM=FB, LRECL $=80, B L K S I Z E=6160$ )

740. //G0.FT21FOO1 DD DSN=\&\&TEMPMC, DISP=(NEW, PASS),

741. I UNIT=SYSDA,SPACE $=(T R K,(1,1))$,

742. I/ ICB $(D S O R G=P S, R E C F M=F B, L R E C L=80, B L K S I Z E=6160$ )

743. //GO.FT22F001 DD DSN=\&\&TEMPNU, DISP $=$ (NEW, PASS),

744. I/ UNIT=SYSDA, SPACE $=(T R K,(1,1))$,

745. I/ DCB=(DSQRG=PS, RECFM=FB, LRECL=80, BLKSIIE=6160)

746. //EO.FT23FOO1 DD DSN=\&\&TEMPCA,DISP=(NEH,PASS),

747. I/ UNIT=SYSDA, SPACE $=(\operatorname{TRK},(1,1))$,

748. $/ / \quad$ DCB $=$ (DSORG=PS, RECFM=FB, LPECL $=80, B L K S I Z E=6160$ )

749. //G0.FT24FO01 DD DSN=\&\&TEMFGA, DISP=(NEW, PASS),

750. I/ UNIT $=S Y S D A, S P A C E=(\operatorname{TRK},(1,1))$,

751. I/ DCE $=(D S O R G=P S, R E C F M=F B, L R E C L=80, B L K S I Z E=6160)$

752. //GO.FT25F001 DD DSN=\&\&TEMPNF, DISP $=(N E W, P A S S)$,

753. II UNIT $=S Y S D A, S P A C E=(T R K,(1,1))$,

754. I/ DCB=(DSORG $=P S$, RECFM=FB, LRECL $=80$, BLKSIZE=6160)

755. $\quad / /$ GO. FT26F001 DD DSN=\&\&TEMPSF, DISP $=(N E W, P A S S)$,

756. I/ UNIT $=$ SYSDA, SPACE $=(T R K,(1,1))$,

757. $/ / \quad D C E=(D S O R G=P S, R E C F M=F B, L R E C L=80, B L K S I Z E=6160)$

758. $/ / 600 . F T 27 F 001$ DD ISN=\&\&TEMPMI, DISP $=$ (NEW, PASS),

759. // UNIT $=$ SYSDA, SPACE $=(T R K,(1,1))$,

760. $/ / \quad D C B=(D S O R G=P S, R E C F M=F B, L F E C L=80, B L K S I Z E=6160)$

761. $/ / 60 . F T 28 F 001$ DD DSN=\&\&TEMPIL, DISP=(NEW, PASS),

762. I/ UNIT $=$ SYSIA, SPACE $=(T R K,(1,1))$,

763. $\quad / / \quad D C B=(D S O R G=P S, R E C F Y=F B, L R E C L=80, B L K S I Z E=6160)$

764. //G0.FT29FOO1 DD DSN=\&\&TEMPIN, DISP=(NEW, PASS),

765. II UNIT=SYEDN, SPACE $=(T R K,(1,1))$,

766. $I / \quad D C B=(D S O R G=P S, R E C F M=F B, L R E C L=80, B L K S I Z E=6160)$

767. //G0.FT30F001 DD DSN=\&\&TEMPWI, DISP=(AEW, PASS),

768. I/ UNIT $=$ SYSDA, SPACE $=(T R K,(1,1))$,

769. I/ $D C B=($ LSORG $=P S$, RECFM $=F B$, LRECL $=80, B L K S I Z E=6160$ )

770. //GO.FT31FOO1 DO DSN=\&\&TEMPEK, DISP=(NEW, PASS),

771. $/ /$ UNIT $=S Y S D A, \operatorname{SPACE}=(\operatorname{TRK},(1,1))$,

772. $\quad / / \quad \triangle C B=(D S O R G=P S, R E C F M=F B, L R E C L=80, B L K S 12 E=6160$ )

773. $\quad / / 60 . F T 32 F 001$ DD DSN=\&\&TEMPWK, DISP=(NEW, PASS),

774. $/ /$ UNIT $=$ SYSDA, SPACE $=($ TRK, $(1,1))$,

775. $/ / \quad D C B=(I I S D R G=F S$, RECFM $=F B, L R E C L=80$, BLKSIZE $=6160$ )

776. $/ / G 0 . F T 33 F 001$ DO DSH $=\& \&$ TEMPET, DISP=(NEW,PASS),

777. // UNIT $=$ SYSDA, SPACE $=(T R K,(1,1))$,

778. $/ / \quad$ DCE $=$ (DSORG=PS, RECFM=FB, LRECL=80, BUKSIZE=6160)

779. $/ / 60 . F T 34 F 001$ DU DSN=\&\&TEMPWT, DISP=(NEW, PASS),

780. // UNIT $=$ SVSDA, SPACE $=(T R K,(1,1))$,

781. I/ ICR $=(D S O R G=P S, R E C F M=F B, L R E C L=80, E L K S I Z E=6160)$

782. //GO.FT3FF001 DD DSN=\&\&TEMPDK, DISP=(NEW, PASS), 
783. I/ UNIT=SYSDA, SEACE $=(\operatorname{TR},(1,1))$,

734. $/ / \quad D C E=(D S O R G=P S, R E C F M=F E, L R E C L=80, B L K S I Z E=6160)$

785. $\quad / / G 0 . F T 36 F 001$ DD DSN=\&\&TEMPMN, DISP =(NEW, PASS),

786. I/ UNIT=SYSDA, SPACE $=\{$ TRK, $(1,1))$,

787. I/ $\mathrm{DCB}=(\mathrm{DSOR} C=P S$, RECFM $=F E, \angle R E C L=80$, BLKSIZE $=6160)$

788. //60.FT37F001 DI DSN=L\&TEMFKN, DISP=(NEW, PASS $)$,

789. I/ UNIT $=\$ Y S D A$, SPACE $=(T R K,(1,1))$,

790. $\quad 1 / \quad D C B=(D S O R G=P S, R E C F M=F B, L R E C L=80, B L K S I Z E=6160)$

791. $/ / 60 . F T 38 F 001$ DD DSN=KSTEMPIA, DISP=(NEW, PASS),

792. $1 /$ UNIT $=$ SYSLA, SPACE $=(T R K,(1,1))$,

793. I/ $D C B=(D S O R G=P S, P E C F M=F B, L R E C L=80, B L K S I Z E=6160)$

794. //GO.FT39FOO1 DD DSN=\&\&TEMPMO, DISP=(NEW, PASS),

795. I/ UNIT $=S Y S D A, S P A C E=\{T R K,(1,1)\}$,

796. I/ $D C B=(D S O R G=P S, R E C F M=F B, L R E C L=80, B L K S I Z E=6160)$

797. $/ / G O . F T 40 F 001$ DD DSN=\&\&TEMPMT, DISP $=$ (NEW, PASS),

799. II UNIT=SYSDA,SPACE $=(T R K,(1,1))$,

799. I/ $D C B=(D S O R G=P S, R E C F H=F B, L R E C L=80$, BLKSIZE $=6160)$

800. //GO.FT41FO01 DD DSN=\&\&TEMPUY, DISP =(NEW, PASS),

801. // UNIT $=$ SYSDA, SPACE $=($ TRK,$(1,1))$,

802. If $D C B=(D S O R G=P S, R E C F M=F B, \angle R E C L=80, B L K S I Z E=6160$ )

803. //GO.FT42FOO1 DD DSN=\&\&TEMPID,DISP=(NEW, PASS),

804. // UNIT=SYSDA, SPACE $=(T R K,(1,1))$,

805. I/ DCE=(DSORG=PS, RECFM=FB, LRECL=80, ELKSIZE=6160)

806. I/GO.FT43F001 DD DSN=\&\&TEMPCO,DISP=(NEW, PASS),

807. I/ UNIT $=$ SYSDA, SPACE $=(T R K,(1,1))$,

808. $/ / \quad D C B=(D S O R G=P S$, $R E C F M=F B, L R E C L=80, B L K S I Z E=6160$ )

809. $/ / 60$. FT 44F001 DD DSN=\&\&TEMPUT, DISP=(NEW, PASS),

810. I/ UNIT=SYSDA, SPACE $=\{T R K,(1,1))$,

811. II ICB $=$ (DSORG =PS, RECFM=FB, LRECL=80, BLKSIZE=6160)

812. //60.FT45FO01 DD DSN=\&4TEMPNV, DISF=(NEW, PASS),

813. // UNIT $=$ SYSDA, SPACE $=(T R K,(1,1))$,

814. I/ DCE=(DSORO=PS, RECFM=FB, LRECL $=80, B L K S I Z E=6160)$

815. $/ / G 0 . F T 46 F 001$ DD DSN=\&\&TEMPAZ, DISP $=(N E W, P A S S)$,

31b. // UNIT=SYSIIR, SPACE $=\{T R K,(1,1))$,

817. I/ $D C E=(D S O R G=P S, R E C F M=F B, L R E C L=80$, BLKSIZE $=6160)$

813. //GO.FT47FOO1 DII ISN=L\&TEMPNA, DISP=(NEW, PASS),

819. I/ UNIT $=S Y S D A, S P A C E=(T R K,(1,1))$,

820. I/ $D C B=$ (DSORG =PS, RECFM=FB, LRECL=80, ELKSIZE $=6160$ )

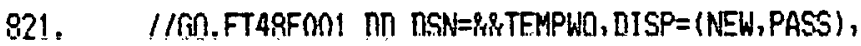

822. I/ UNIT $=$ SYSDA, SPACE $=($ TRK,$(1,1))$,

823. I/ DCE $(D S O R G=P S, R E C F M=F B, L R E C L=80, B L K S I Z E=6160)$

824. $/ / G 0 . F T 49 F 001$ DD DSN=\&\&TEMPCN, DISP $=(N E W, F A S S)$,

825. I/ UNIT $=$ SYSDA, SPACE $=(T R K,(1,1))$,

826. I/ $D C B=(D S O R G=P S, R E C F M=F B, L R E C L=80, B L K S I Z E=6160)$

827. $/ / 60$. FT50FOO1 DO DSN=\&\&TEMPCS, DISP=(NEW, FASS),

828. II UNIT=SYSDA, SPACE $=(T R K,(1,1))$,

829. I/ DCE $=(D S O R G=P S, R E C F M=F B, L R E C L=80, B L K S I Z E=6160)$

830. //GO.FT5!FOO1 DD DSN=\&\&TEMPEP, DISF=(NEW,PASS),

831. I/ UNIT=SYSDA, SPACE $=\{$ TRK, $(1,1))$,

832. $/ / \quad D C E=(D S O R C=P S, R E C F M=F B, L R E C L=80, B L K S I Z E=6160)$

833. $/ / 60$. FTS2FOO1 DI DSN=\&\&TEMPJY, DISP =(NEH, PASS),

834. I/ UNIT $=S Y S D A, S F A C E=(T K K,(1,1))$, .

935. I/ DCB=(IDSORG=PS, RECFM=FB, LRECL =80, ELKSIIE=6160)

s.6. //GD. FTE?FOMI DI DSN=\&\&TEMPUP, DISP =(NEW, PASS),

837. I/ UNIT=SYSEA, SPACE $=\{T R K,(1,1))$,

838. I/ $D C B=(D S O R G=P S, R E C F M=F B, L R E C L=80, B L K S I Z E=6,160)$ 
839. //GO.FT54F001 DD ISN=\&\&TEMPNW, DISP =(NEW, PASS),

840. // UNIT $=$ SYSLA, SPACE $=(T R K,(1,1))$,

841. // ICE = (ISORG $=P S$, RECFM=FB, LRECL $=80$, ELKSIIE $=6160)$

842. //GO.FT55F001 DI DSN=\&\&TEMPSW, DISP =(NEW, PASS),

843. I/ UNIT $=S Y S D A, G P A C E=i T R K,(1,1)$,

844. I/ ICB $=(U S O R G=P S, R E C F M=F B, L R E C L=80$, BLKSIZE=6160)

845. //GO.FTSEFOO1 DO DSN=E\&TEMPVA, DISF=(NEW, PASS),

846. I/ UNIT $=S Y S D A, S P A C E=(T R K,,(1,1))$,

847. I/ ICE=(DSORG=PS, RECFM=FE,LRECL=80, BLKSIZE=6160)

848. //G0.FT57F001 DD DSN=\&\&TEMPDM, [IISP=(NEW, PASS),

849. // LNIT $=$ SYSIA, SPACE $=(T R K,(1,1))$,

850. $/ 1 \quad D C B=(D G O R G=P S, R E C F M=F B, L R E C L=80, B L K S I Z E=6160)$

851. $/ / G 0 . F T 58 F 001$ DO DSN=\&\&TEMPON, DISP=(NEW, PASS),

852. // UNIT $=S Y S D A$, SPACE $=(T R K,(1,1))$,

853. $/ / \quad D C B=(D S O R G=P S, R E C F M=F B, L R E C L=80, B L K S I Z E=6160)$

854. //GO.FT59F001 DD DSN=\&\&TEMPOM, DISP=(NEW, PASS),

855. // UNIT $=S Y S D A$, SPACE $=(T R K,(1,1))$,

956. I/ DCB $=($ IISORC $=P S$, RECFM $=F B, L R E C L=80, B L K S I Z E=6160)$

357. //GO.FT6OFOO1 DD DSN=\&\&TEMPOS, DISP=(NEW, PASS),

858. // UNIT $=S Y S D A, S P A C E=(T R K,(1,1))$,

859. I/ DCB $=(D S O R G=P S, R E C F M=F B, L R E C L=80, B L K S I Z E=6160)$

S60. //GO. FT61F001 DD DSN=\&\&TEMPAM, IISP = (NEW,PASS),

861. // LNIT $=S Y S D A, S F A C E=(T R K,(1,1))$,

862. $/ / \quad$ ICE $=$ (DSORI $=P S, R E C F M=F B, L R E C L=80$, BLKSIZE $=6160$ )

863. $/ / 60 . F T 62 F 001$ DD DSN=\&\&TEMPTX,DISP $=(N E W, P A S S)$,

864. // UNIT $=S Y S D A, S P A C E=(T R K ;(1,1))$,

865. I/ DCB=(DSORG=PS, RECFM=FB, LRECL=80, BLKSIZE=6160)

866. //G0. FT63F001 BD OSN=L\&TEMPA0,DISP=(NEW, PASS),

867. I/ UNIT $=$ SYSDA, SPACE $=(T R K,(1,1))$,

868. // ICE= (DSORO=PS, RECFM=FB, LRECL=80, BLKSIZE $=6160$ )

869. $/ / 60 . F T 64 F 001$ DI DSN=\&\&TEMPNA, DISF=(NEW, FASS),

870. I/ UNIT $=$ SYSDA, SPACE $=(T R K,(1,1))$,

871. I/ DCE $($ USCRG $=F S, R E C F M=F B, L R E C L=80$, BLKSIZE $=6160)$

872. //WELD EXEC PGM=IEFBR14, REGION=1OK

873. //DD1 DO DSN=CN6376. MI2. NURES.FORM1M.FDS7S, DISF=(MOD, DELETE),

874. I/ UNIT=[ASU, SPACE $=($ TRK, $(7,1,3))$

875. I/DELII EXEC PGM=IEFRR14, REGION=1OK

876. //DD1 DII DSN=CN6376. MI2. NLREG. FORM1M. PDS78, DISP=(NEW, CATLG),

877. $/ 1 \quad$ LNIT= LASD,

878. $/ /$ SPACE $=(T F K,(7,1,3)), D C E=(D S O R G=P 0$, RECFM $=F B, B L K S I Z E=6.160, \angle R E C L=80)$

879. //GENER EXEC PGM=IEBGENER

380. I/SYSPRINT DD DUMMY

881. //SYSUT1 DII DSN=\&\&TEMPMV, DISP=(OLD, DELETE)

882. //SYSUT2 DD DSN=CN6376. MI2. NUREG. FORM1M. PDS78(MV), DISP=(OLD, KEEP)

383. //SYSIN DII IUMMY

Q84. //GENER EXEC. PGM=IEBGENER

885. ISYSPRINT DD DUMMY

886. //SYSUT1 ID ISON=\&\&TEMPMC, DISP=(OLD, DELETE)

337. I/SYSUT2 DD IGN=CNS376. MI2. NUREG. FORM1M. PDS78(MC), DISP=(OLD, KEEP)

888. I/SYSIN DO DUMMY

889. //GENER EXEC PGM=IEEGENER

390. I/SYSPRINT DD DLMMY

891. //SYSITI DII DSN=\&\&TEMPNH, DISP=(OLD, DELETE)

892. //SYSUT2 DII DSN=CN6376. MIZ. NLREG. FORMIM. PDS78(NU1), UIISP=(OLD, KEEP)

893. I/SYSIN DII DUMMY

894. //GENER EXEC. PGM=IEBGENER 
395. I/SYSPRINT DD DUMMY

896. I/SYSUT1 UII DSN=\$RTEMFCA, DISF=(OLD, DELETE)

897. //SYSUT2 DII DSN=CN6376. MI2. NUREG. FORM1M. PDS78(CA), DISP=(OLD, KEEP)

898. //SYSIN DD DUMYY

899. //GENER EXEC PGH=IEBGENER

900. I/SYSPRINT DO DUMAY

901. //SYSUT1 DUI USN=\&\&TEMPGA, DISP=(OL[I, DELETE)

902. I/SYSUT2 DII DSN=CNE376. MI2. NUREG. FORM1M. PDS78(GA), DISF=(OLD, KEEF)

903. I/SYSIN DO DUMMY

904. I/GENER EXEC PGM=IEBGENER

905. I/SYSPRINT DD DUMMY

906. I/SYSUTI DD DSN=\&\&TEMPNF, DISP=(OLD, DELETE)

907. //SYSUT2 DD DSN=CN6376. MI2. NUREG. FORM1M. PIS78(NF), DISP=(OLD, KEEP)

908. I/SYSIN DD DUMMY

909. //GENER EXEC PGM=IERGENER

910. I/SYSPRINT DII DUMMY

911. //SYSUTI DD DSN=\&\&TEMPSF, DISP=(OLD, DELETE)

912. //SYSUT2 DD DSN=CNE376. MI2. NUREG. FORM1M. PDS78(SF), DISP=(OLD, KEEP)

913. I/SYSIN DO DUPSYY

914. I/GENER EXEC PGM=IEBGERER

915. I/SYSPRINT DO DUMHY

916. I/SYSUTI DU DSN=\&RTEMPMI, DISP=(OLI, DELETE)

917. //SYSUT2 DD DSN=CN6376. MI2. NUREG. FORM1M. PDS78(MI), DISP=(OLD, KEEP)

913. //SYSIN DD DUMMY

919. //GENER EXEC PGM=IEBGENER

920. I/SYSPRINT DO DUMMY

921. I/SYSUT1 DD DSN=\&4EMPIL, DISP=(OLD, DELETE)

922. //SYSUT2 DD DSN=CN6376.M12. NUREG. FORMIM. PDS78(IL), DISP=\{OLD, KEEP)

923. I/SYSIN DD DUMMY

924. I/GENER EXEC PGM=IEBGENER

925. //SYSPRINT DO DUMMY

926. I/SYSUTI DO ISN=\&\&TEMPIN, DISP=(OLD, DEETE)

927. //SYSUT2 DD IISN=CN6376. MI2. NUREG. FOFMIM. PIS7B(IN), DISP=(OLD, KEEP)

928. //SYSIN DO RUMYY

92\%. I/GENER EXEC PGM=IEBGENER

930. I/SYSPRINT DD DUMAY

931. I/SYSUTI DD IISN=\&:TEMPUI, UIST=(OLD, DELETE)

932. (/SYSIT2 DD DSN=CNL376. MI2. NUREG. FORM1M. PIS78(WI), DISP=(OLD, KEEP)

933. I/SYSIN DD DUMMY

934. I/GENER EXEC PGM=IEBGENER

935. //SYSPRINT DD DUMMY

936. //SYSUT1 DD DSN=\&\&TEMPEK, DISP=(OLD, DELETE)

937. I/SYSUT2 DD DSN=CNG376. MI2. NUREG. FORMIM. PDS78(EK), DISP=(OLD, KEEP)

938. //SYSIN DD DUMYY

93\%. //GENER EXEC PGM=IEBGENER

940. I/SYSPRINT IDD DLMMY

941. //SYSUT1 OD ISN=\&\&TEMPWK, DISP $=\{$ OLII, DELETE)

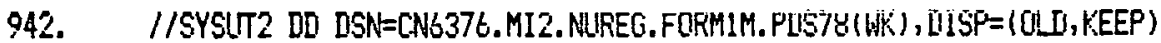

943. I/SYSIN DN DUPMY

944. I/GENER EXEC PGY=IEBGENER

945. //SYSPRINT DD DAMMY

946. I/SYSUT1 DD DSN=\&\&TEMPET, DISF=(OLD;DELETE)

947. //SYSUT2 DD ISN=CN6376. MI2. NUREG. FORM1M. PDS78(ET), DISP=(OLD, KEEP)

948. I/SYSIN DII DUMMY

94\%. //GENER EXEC PGM=IEBGENER

950. //SYSPRINT DD DAMMY 
951. //SYSUT1 DO DSN=R\&TEMFWT, DISP=(OLD, DELETE)

952. //SYSUT2 DD DSN=CN6376. MI2. NUREG. FORM1M. PDS78(WT), IIISF=(OLD, KEEP)

953. //SYSIN DU DUMMY

954. //GENER EXEC PGM=IEBGENER

955. //SYSPRINT DO DUMYYY

956. - //SYSUT1 OL ISN=\&\&TEMFDK, DISP $=$ (OLD, DELETE)

957. //SYSIT2 DII DSN=CN6376. MI2. AUREG. FOFM1M. PDS78(DK), DISP=\{OLD, KEEP)

958. I/SYSIN DO DUMMY

559. //GENER EXEC: PGM=IEBGENER

960. I/SYSPRINT DD IUUMYY

961. //SYSUT1 DD DSN=\&\&TEMPMN, DISP=(OLD, DELETE)

962. //SYSUT2 DO DSN=CNG376. MI2. NLREG. FORM1M. PDS78(NN), DISP=(OLD, KEEP)

963. I/SYSIN DD DUMMY

964. I/GENER EXEC PGM=IEBGENER

965. I/SYSPRINT DD DUMMY

966. I/SYSUT1 DD DSN=\&\&TEMPKN, DISP=(OLD, DELETE)

967. //SYSUT2 DD DSN=CN6376. MI2. NUREG. FORM1M. PDS78(KN), DISP=(OLD, KEEP)

968. I/SYSIN DO DUPAYY

969. I/GENER EXEC PGT=IEBGENER

970. IISYSPRINT DD DUMYY

971. . //SYSUT1 DD DSN=\&LTEMPIA, DISP=(OLD, DELETE)

972. //SYSUT2 DO DSN=CN6376. MI2. NUREG. FORMIM. PDS78(IA), DISP=(OLD, KEEP)

973. I/SYSIN DD DUMYY

974. I/GENER EXEC PGM=IEBGENER

975. I/SYSPRINT DO DUMMY

976. I/SYSUIT DD DSN=\&\&TEMPMO,DISP=(OLD,DELETE)

977. //SYSUT2 DD DSN=CN6376. MI2. NUREG. FORMIM. PIS78(MO),DISP=(OLD, KEEP)

978. I/SYSIN DD DUMMY

979. I/GENER EXEC PGM=IEBGENER

980. - I/SYSPRINT DD DUMMY

901. I/SYSUT1 DO DSN=\&\&TEMPMT, UISP=(OLD,DELETE)

982. I/SYSUT2 DD DSN=CNG376. MI2. NUREG. FORM1M. PDS78(MT), DISP=(OLD, KEEP)

983. I/SYSIN DO DUMMY

984. //GENER EXEC. PGM=IEBGENER

985. //SYSPRINT DD DUMYY

986. //SYSUT1 DD DSN=\&\&TEMPWY, DISP=(OLD, DELETE)

987. //SYSUT2 DD DSN=CN6376. MI2. NUREG. FORN1M. PDS78(WY), DISP=(OLD, KEEP)

988. I/SYSIN DD DUMYY

939. I/GENER EXEC PGM=IEBGERER

990. I/SYSPRINT DD DUMAYY

991. I/SYSUTI DD DSN=\&\&TEMPID,DISP=(OLD, DELETE)

992. I/SYSUT2 DD DSN=CN6376. MI2. NUREG. FORM1M. PDS78(ID), DISP=(OLD, KEEP)

993. I/SYSIN DD DUMYYY

994. I/GENER EXEC PGM=IEBGENER

$995 . \quad$ //SYSPRINT DO DUYAY

996. //SYSUT1 DU DSN=\&\&TEMPCO,DISP=(OLD, DELETE)

997. I/SYSUT2 DI ISN=CN6376. MI2. NUREG. FOSMIM. PDS78 (C0), DISP=(OLII, KEEP)

998. //SYSIN DO DIMMY

99. I/GENER EXEC: PGM=IERGENER

1000. //SYSPRINT DL DUMMY

1001. I/SYSUT1 DD DSN=\&\&TEMPUT, DISP $=$ (OLD, DELETE)

1002. I/SYSUT2 DD DSN=CN6375. MI2. NUREG. FGRMIM, PDS78(UT), DISP=(OLD, KEEP)

1003. I/SYSIN DD DIMMY

1004. //GENER EXEC. PGH-IGIGENER

1005. //SYSPRINT DD DUMMY

1006. I/SYSUT1 DD DSN=\&\&TEMPNV, DISF=(OLD, DELETE) 
1007. I/SYSUT2 DO DSHECNG376. MI2. NUREG. FORM1M. PDS78(NV), DISP=(OLD, KEEP)

1008. I/SYSIN DD DUMMY

1009. //GENER EXEC PGM=IEBGENER

1010. I/SYSPRINT DI DUMMY

1011. //SYSUT1 DD DSN=:\&TEMPAZ, LIISP=(OLD, DELETE)

1012. //SYSUT2 DI DSN=CNG376. MI2. NUIREG. FORMIM. PDS78(AZ), DISP=10LD, KEEP)

1013. //SYSIN DD DIMMYY

1014. //GENER EXEC PGM=IEBGENER

1015. //SYSPRINT DII DUMMY

1016. //SYSUT1 DO DSN=L\&TEMPNM, DISP = (OLD, DELETE)

1017. //SYSUT2 DI DSN=CNG376. MI2. NUREG. FORMIM. PDS78(NM), DISP=(OLD, KEEP)

1018. //SYSIN DD DUMMY

1019. //GENER EXEC PGY=IEBGENER

1020. I/SYSPRINT DO DUMMY

1021. //SYSUT1 DO DSN=\&\&TEMPWO, DISP=(OLD, DELETE)

1022. //SYSUT2 DD DSN=CN6376. MI2. NUREG. FORM1M. PDS78(WO), DISP $=$ (OLD, KEEP)

1023. //SYSIN DD DUMMY

1024. //GENER EXEC. PGM=IEBGENER

1025. I/SYSPRINT DD DUMYY

1026. I/SYSUT1 DD DSN=\&\&TEMPCN, DISP=(OLD, DELETE)

1027. I/SYSUT2 DD DSN=CNE376. MI2. NUREC. FORM1M. PDS78(CN), DISP=(OLD, KEEP)

1028. I/SYSIN DD DUMMY

1029. //GENER EXEC PGM=IEBGENER

1030. //SYSPRINT DD DUMMY

1031. //SYSUT1 DD DSN=\&\&TEMPCS, DISP=(OLD, DELETE)

1032. //SYSUT2 DD DSN=CN6376.MI2. NUREG.FORMIM. PDS78(CS), DISP=(OLD, KEEP)

1033. I/SYSIN DD DUMMY

1034. //GENER EXEC PGM=IEBGENER

1035. //SYSPRINT DD DUMMY

1036. I/SYSUT1 DD DSN=\&\&TEMPEP, DISP=(OLD, DELETE)

1037. I/SYSUT2 DO DSN=CN6376. MI2.NUREC. FORMIM. PDS7P(EP), DISP=(OLD, KEEP)

1038. I/SYSIN DD DUMMY

103\%. //GENER EXEC PGM=IEBGENER

1040. //SYSPRINT DO DUMMY

1041. I/SYSIT1 DO ISN=\&\&TEMPJN, DISF=(OLI, IELLTE)

1042. //SYSUT2 DU DSN=CN6376. MI2. NUREG.FORMIM. PDS78 (JY), DIISP=(OLD, KEEP)

1043. I/SYSIN DD DUMMY

1044. //GENER EXEC. PGM=IEBGENER

1045. //SYSPRINT DD DUMMY

1046. //SYSUT1 DD DSN=\&\&TEMPWP, DISP = (OLD, IELLETE)

1047. //SYSUT2 DO DSN=CNE376. MI2. NUREG. FORM1M. PDS78(WP), DISP=(OLD, KEEP)

1048. I/SYSIN DD DUMMY

1049. //GENER EXEC PGM=IEBGENER

1050. //SYSPRINT DD DUMMY

1051. //SYSUT1 DD DSN=\&\&TEMPNW, DISP =(OLD, DELETE)

1052. I/SYSIJT2 DD DSN=CNG376. MI2. NUIREG. FORM1M. PDS78(NN), DISP=(OLD, KEEP)

1053. I/SYSIN DD DUMMY

1054. //OENER EXEC. FQMะIEEOENER

1055. //SYSPRINT DD RUMMY

1056. I/SYSUT1 DD DSN=\&\&TEMPSW, DISP=(OLD, DELETE)

1057. //SYSUT2 DD DSN=CNE376. MI2. NLIREG. FORM1M. FDS78(SW), DISF=(OLD, KEEP)

1058. //SYSIN DD DUMMY

1059. //GENER EXEC: PGM=IEBGENER

1060. //SYSPRINT DD DUMMY

1061. //SYSUT1 DD DSN=\&\&TEMPVA, DISP $=\{$ OLD, IIELETE $)$

1062. //SYSUT2 DD USN=CN6376. MI2. MUREG. FORMIM. FIS78(VA), [IISP=(OLD, KEEP) 


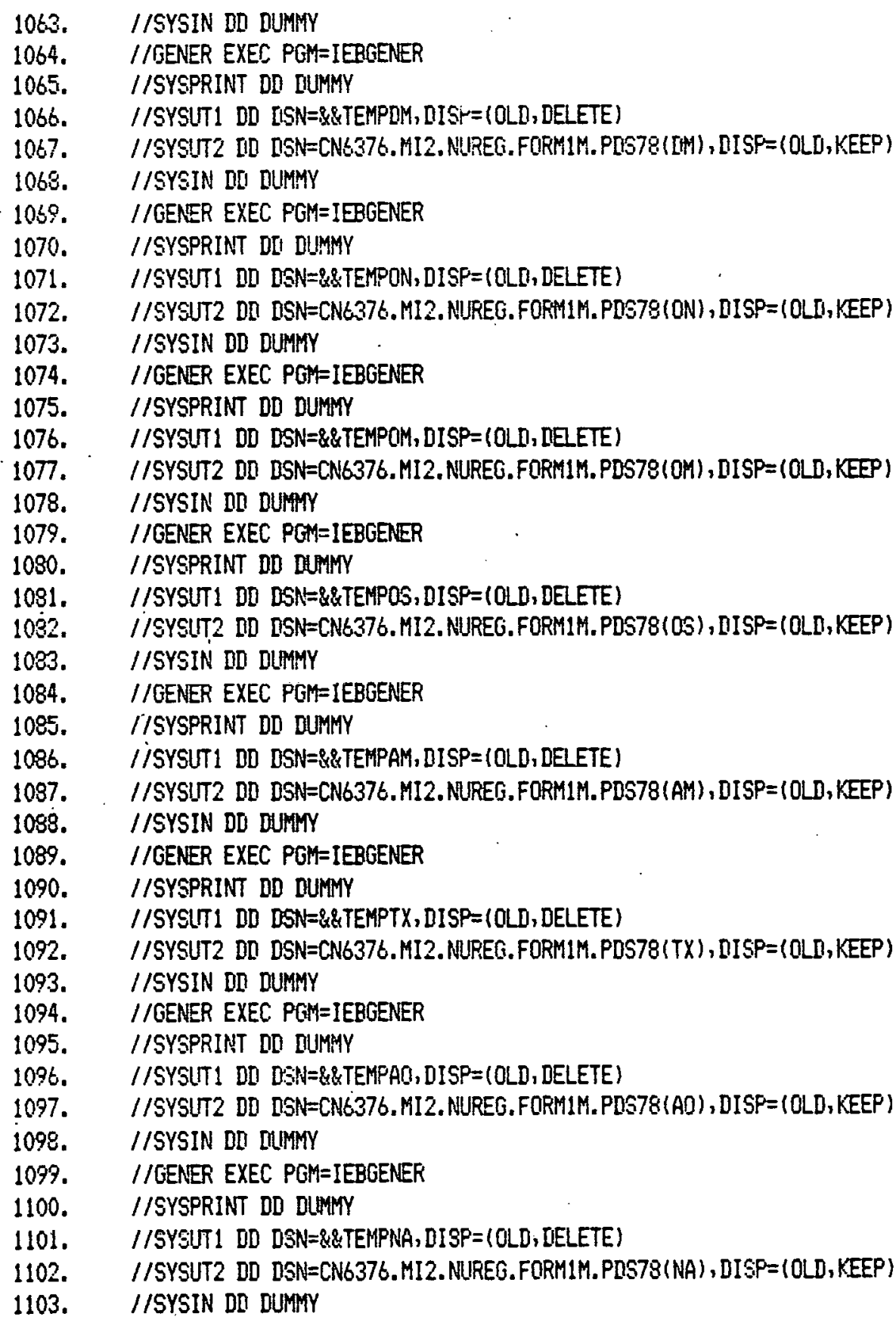

